$10 / 3.24-97850$

SAND97-8220 • UC-404

Unlimited Release

- $\quad$ Printed February 1996

$5 A N D-97-8220$

1970.19

\title{
The Development of Lightweight Hydride Alloys Based on Magnesium
}

- S. E. Guthrie, G. J. Thomas, W. Bauer, N. Y. C. Yang

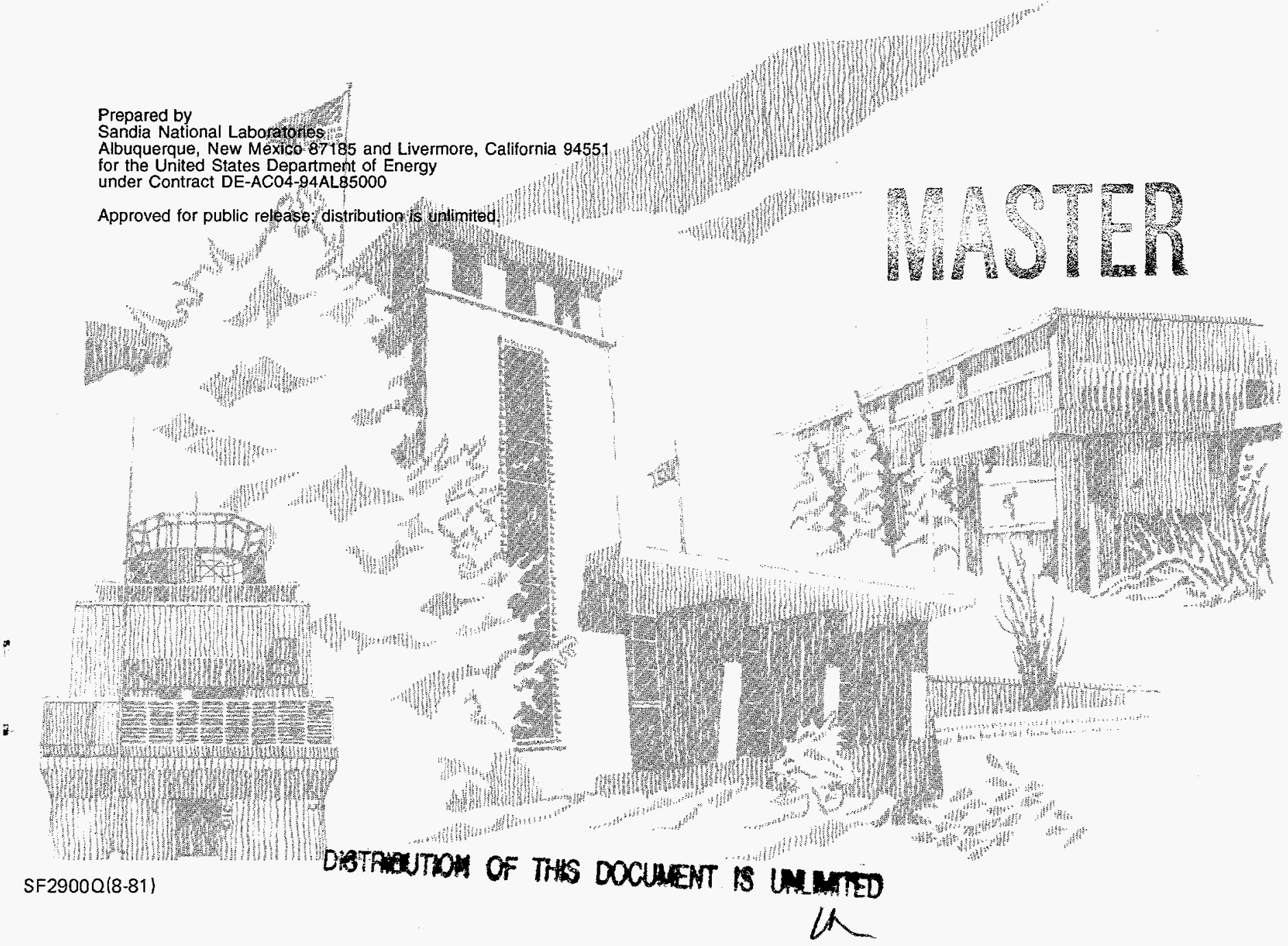


Issued by Sandia National Laboratories, operated for the United States Department of Energy by Sandia Corporation.

NOTICE: This report was prepared as an account of work sponsored by an agency of the United States Government. Neither the United States Government nor any agency thereof, nor any of their employees, nor any of the contractors, subcontractors, or their employees, makes any warranty, express or implied, or assumes any legal liability or responsibility for the accuracy, completeness, or usefulness of any information, apparatus, product, or process disclosed, or represents that its use would not infringe privately owned rights. Reference herein to any specific commercial product, process, or senvice by trade name, trademark, manufacturer, or otherwise, does not necessarily constitute or imply its endorsement, recommendation, or favoring by the United States Government, any agency thereof or any of their contractors or subcontractors. The views and opinions expressed herein do not necessarily state or reflect those of the United States Government, any agency thereof, or any of their contractors or subcontractors.

This report has been reproduced from the best available copy.

Available to DOE and DOE contractors from:

Office of Scientific and Technical Information

P.O. Box 62

Oak Ridge TN 37831

Prices available from (615) 576-8401, FTS 626-8401.

Available to the public from:

National Technical Information Service

U.S. Department of Commerce

5285 Port Royal Rd.

Springfield, VA 22161 


\section{DISCLAMMER}

Portions of this document may be illegible in electronic image products. Images are produced from the best available original document. 
SAND $97-8220$

UC-404

Unlimited Release

Printed February 1996

\title{
THE DEVELOPMENT OF LIGHTWEIGHT HYDRIDE ALLOYS BASED ON MAGNESIUM
}

\author{
S. E. Guthrie, G. J. Thomas, N. Y. C. Yang \\ Surface and Microstructure Research Department \\ and \\ W. Bauer \\ Materials \& Combustion Technology Department \\ Sandia National Laboratories/California
}

\begin{abstract}
The development of a magnesium based hydride material is explored for use as a lightweight hydrogen storage medium. It is found that the vapor transport of magnesium during hydrogen uptake greatly influences the surface and hydride reactions in these alloys. This is exploited by purposely forming near-surface phases of $\mathrm{Mg}_{2} \mathrm{Ni}$ on bulk $\mathrm{Mg}-\mathrm{Al}-\mathrm{Zn}$ alloys which result in improved hydrogen absorption and desorption behavior. Conditions were found where these nearsurface reactions yielded a complex and heterogeneous microstructure that coincided with excellent bulk hydride behavior. A Mg-Al alloy hydride is reported with near atmospheric plateau pressures at temperatures below $200^{\circ} \mathrm{C}$. Additionally, a scheme is described for low temperature in-situ fabrication of $\mathrm{Mg}_{2} \mathrm{Ni}$ single phase alloys utilizing the high vapor pressure of $\mathrm{Mg}$.
\end{abstract}




\section{ACKNOWLEDGMENTS}

The authors thank Dr. J. R. Spingarn and Dr. E. Lavernia for their helpful discussions and assistance in securing and fabricating sample materials. The authors also acknowledge the helpful discussions with Dr. Gary Soundrock, SunaTech Inc. The authors wish to acknowledge the assistance of A. M. Sieber in the hydride measurements; C. K. Rood and A. D. Gardea for their assistance in the microscopy measurements and preparations; and, K. D. Stewart for his assistance in mechanical design and assembly. We gratefully acknowledge the support of the LDRD Office which has led to a continuing program in this area in support of the U.S. DOE Hydrogen Program. 


\section{CONTENTS}

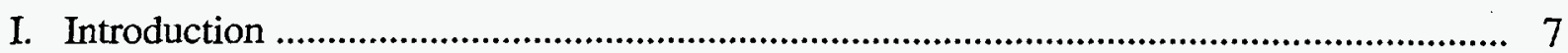

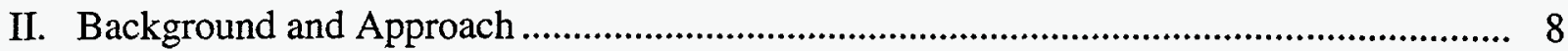

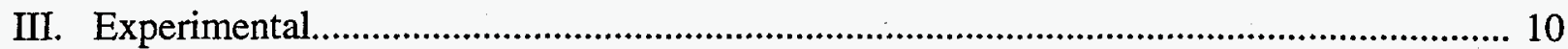

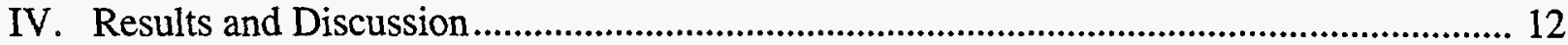

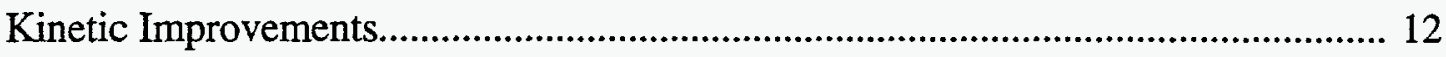

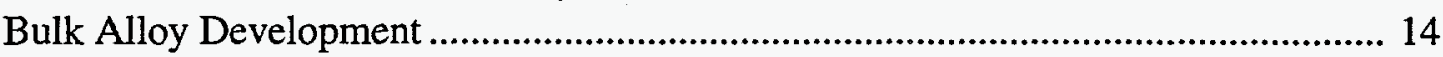

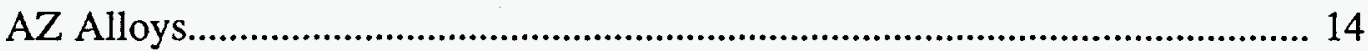

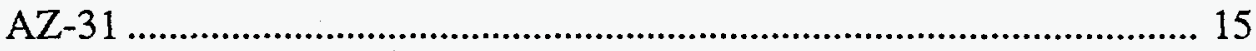

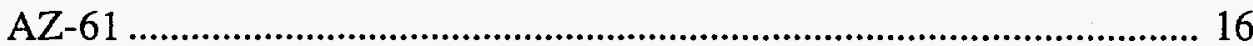

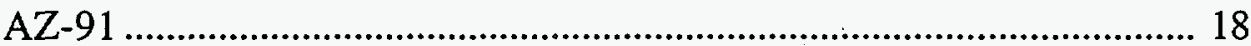

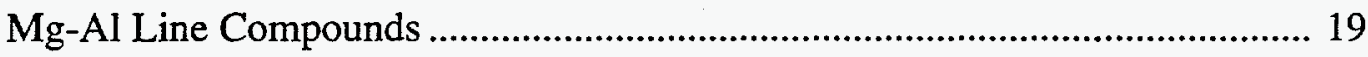

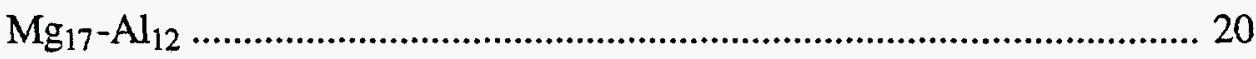

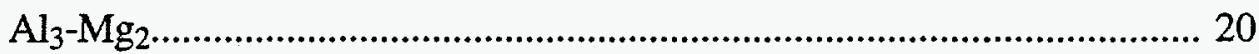

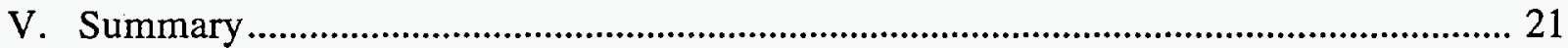

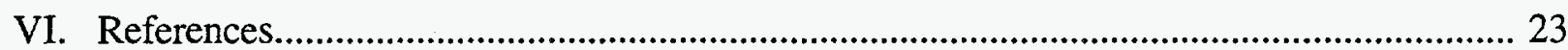

FIGURES

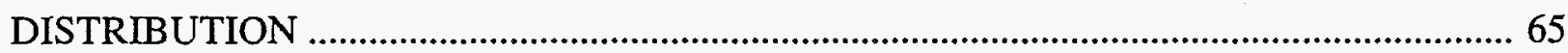




\section{THE DEVELOPMENT OF LIGHTWEIGHT HYDRIDE ALLOYS BASED ON MAGNESIUM}

\section{Introduction}

It has long been recognized that hydrogen is a clean energy carrier and is potentially available from renewable resources domestically. ${ }^{1-2}$ However, a major technological barrier to hydrogen utilization, for vehicular applications in particular, is the need for a lightweight, energy dense storage medium. ${ }^{3}$ One approach to hydrogen storage is the use of metal hydrides to bind hydrogen chemically in a dense solid phase. There is ample literature that shows high volumetric densities have been achieved using this approach. ${ }^{46}$ In addition, there are inherent safety advantages, including low pressure operation and self-limiting hydrogen release, that make hydrides an attractive storage medium. However, two factors critically limit this use of hydrides: hydrogen release kinetics and weight. Hydride kinetics are dictated by the speed of the forward and reverse hydriding reactions. These reactions must be rapid enough to meet the demands of refueling operations as well as those of the primary power source. The second limitation concerns the additional weight of the materials that form the hydride. The ratio of hydrogen weight to hydride weight expressed as a percentage $\left(\mathrm{H}_{2} \mathrm{wt} . \%\right)$ is an important parameter to judge the storage efficiency. A higher percentage is more desirable for lightweight applications.

Transition metals and rare earth elements have been shown to produce hydride forming alloys which can store and release hydrogen rapidly near room temperature; however, these are generally heavy, with 1-2 $\mathrm{H}_{2}$ wt.\% achievable. The low $\mathrm{Z}$ hydride forming elements $(\mathrm{Li}, \mathrm{Mg}, \mathrm{Na}, \mathrm{Al})$ are more attractive (4-12 $\mathrm{H}_{2}$ wt.\%), but tend to form line compounds that are largely ionic or covalently bonded. The high stability of these bonds complicates the retrieval of hydrogen from the hydride by requiring relatively high operating temperatures $\left(>300^{\circ} \mathrm{C}\right)$. Nevertheless, these hydrides, in particular $\mathrm{MgH}_{2}$, probably offer the only solution for achieving a practical, lightweight hydride storage material. Improvements in their performance could affect the utilization of hydrogen in mobile or vehicular applications.

This study was aimed at exploring lightweight magnesium-based hydride alloys with the goal of overcoming the shortcomings of magnesium hydride: poor hydrogen kinetics and high temperature operation for near atmospheric hydrogen overpressures. Our approach is described in the following section along with the criteria used in the selection of materials for this study. Following the approach section, a description of the experimental techniques and general procedure is provided. The experimental data and discussion for each material system studied is then presented. 


\section{Background and Approach}

Hydrides are typically characterized by a metal-hydrogen phase diagram. An example is shown in Figure 1. It is assumed that the hydride phase is in equilibrium with a surrounding hydrogen gas, so that the gas either leaves or enters the solid to maintain thermodynamic equilibrium. Therefore, the phase behavior or pressure-concentration-temperature (PCT) data is represented by plotting the equilibrium gas pressure versus the hydrogen concentration in the solid at different temperatures. Due to a hysteresis effect that separates the equilibrium absorption and desorption conditions, two data sets or curves result at each temperature. Equilibrium is always observed at a higher pressure during absorption. These PCT plots have a characteristic shape dominated by a large constant pressure plateau in which the hydride and metal phases coexist in the solid over an extended range of hydrogen concentration (Figure 1). The plateau is bounded at low hydrogen compositions (< $0.06 \mathrm{H} / \mathrm{M}$ ) by a randomly distributed interstitial solution phase $(\alpha)$ and at higher concentrations by an ordered hydride phase $(\beta)$. Much of the practical information about a hydride concerns the magnitude and breadth of this plateau. In practice, the plateau may have a finite slope and there may be more than one plateau. Nevertheless', on a log scale the pressure plateau is relatively flat and in this form it is proportional to the hydrogen free energy of the system (metal + gas). Typically, the pressure at the center of the plateau for each isotherm can be used to relate thermodynamic variables to the plateau-temperature behavior via the van't Hoff relationship:

$$
\ln \left(P / P_{o}\right)=\Delta H / R T-\Delta S / R
$$

In the equation, $R$ is the gas constant and $P_{0}$ is the atmospheric reference pressure in the appropriate units. Thus, over a limited temperature range, a plot of $\log$ pressure $(P)$ versus reciprocal temperature $(T)$ is a straight line and the enthalpy $(\Delta \mathrm{H})$ and entropy $(\Delta \mathrm{S})$ of formation may be obtained without recourse to calorimetry. In this study, these van't Hoff plots will be used to supplement PCT plots and summarize the hydride behavior of different materials. The shortcomings of this procedure have been discussed by Flanagan, et al. ${ }^{7}$

Numerous correlations between these thermodynamic functions and various parameters of hydrides have been asserted in an effort to rationalize the development of new hydrides. The models range from largely empirical correlations, such as the inverse relationship between the thermal stability of the hydride and the heats of formation of its components ("the rule of reversed stability"), ${ }^{8}$ to semi-empirical theoretical models for calculating the enthalpies of formation.9-11 They have been reasonably successful at establishing a qualitative microscopic understanding of the effects of interstitial hole size, ${ }^{12}$ first and second nearest neighbor interactions,,$^{13}$ and electronic factors such as band filling in metallic hydrides. ${ }^{14}$ However, a quantitative predictive capability for modifying hydride properties is not yet available, particularly for ionic and covalent hydrides.

Previous studies have approached Mg-based alloy development by a largely empirical process in which numerous alloying components were tested to characterize their effects on the base $\mathrm{Mg}$ hydride behavior. ${ }^{15-16}$ Selvam has reviewed much of the previous work that followed from these earlier studies. ${ }^{17}$ Although these studies present an invaluable knowledge base, they have not yielded a clear rationale to follow for further improvement of the alloy behavior. 
Part of the difficulty in deducing such a rationale for magnesium-based hydrides lies in the effort to develop a single homogeneous alloy to address both the equilibrium and kinetic problems. A different strategy would be to separately optimize these objectives in the component phases of a heterogeneous material to achieve a favorable, combined effect. The promise of such an approach lies in the recognition that the equilibrium properties of the hydride, reflected in the pressure and temperature, are largely dictated by the thermodynamics of the bulk properties of the alloy while the kinetic properties are affected by processes that occur in the near-surface and gas/solid interfaces. Thus, the formation of a heterogeneous material with separate surface and bulk phases may provide a way to improve both kinetic and equilibrium properties.

The approach used in this study was to explore the extent to which a heterogeneous Mg-based material could be developed with separately optimized phases. The most direct way to demonstrate this is to start with a bulk magnesium alloy with acceptable equilibrium properties of pressure and temperature and provide a thin surface alloy phase with the requisite kinetic behavior. Given no internal barriers to hydrogen transport between the phases, a storage material could be developed which would yield a higher operating pressure at a lower temperature without excessive weight penalties over those of magnesium hydride. Since the object of the study was to demonstrate the effectiveness of this two-fold approach, a broad survey of potential elemental additions was not attempted. Rather, the scope of the study was limited to $\mathrm{Mg}-\mathrm{Al}$ based alloys for the bulk investigations and $\mathrm{Ni} / \mathrm{Mg}-\mathrm{Ni}$ alloys for the surface kinetic improvements. These choices were based on earlier studies reported in the literature and are discussed below.

A less stable bulk hydride phase is clearly necessary to improve the pressure/ temperature behavior of magnesium hydride. Evidence for such reduced stability was reported earlier by Reilly and Wiswall ${ }^{18}$ and Nachman and Rohy ${ }^{16}$ for the intermetallic Mg-Al line compounds (Figure 2). Unfortunately, these binary compositions exhibited poor hydrogen kinetics below $300^{\circ} \mathrm{C}$. Nevertheless, one alloy $(0.55 \mathrm{Al})$ in this plot can be observed to exhibit excellent pressure behavior, especially if it could be extended to lower temperatures. Since neither $\mathrm{Mg}$ nor $\mathrm{Al}$ provide very active surfaces for hydrogen dissociation, the alloy may be limited at lower temperatures by the surface. Other results suggested that these alloys may, be limited by a decomposition transformation to magnesium hydride and aluminum. ${ }^{19-20}$ This would be consistent with the high mobility of the constituents at temperatures as low as $200^{\circ} \mathrm{C} .{ }^{21}$ Similar phase changes occur in $\mathrm{Mg}-\mathrm{Al}$ structural alloys as the temperature is increased from room temperature. ${ }^{22}$ The mechanical properties in these alloys are improved by the addition of $\mathrm{Zn}$ which limits the grain boundary diffusion of $\mathrm{Al}$ by forming ternary phases. In this regard, the addition of $\mathrm{Zn}$ has become a standard industrial practice for the fabrication of $\mathrm{Mg}$ - $\mathrm{Al}$ cast alloys. The potential role of $\mathrm{Zn}$ in $\mathrm{Mg}$ alloy hydrides was explored in this study.

The hydrogen-metal reaction at the surface is an important factor in limiting kinetic rates. Surface magnesium phases not only form very stable oxide barriers to initial hydrogenation, ${ }^{23}$ but also do not easily absorb hydrogen even in an unoxidized state. ${ }^{24} \mathrm{The}_{2}$ molecular dissociation into $\mathrm{H}$ atoms limits the surface boundary condition for subsequent bulk diffusion. A further complication in kinetics results from precipitation of the hydride phase in the near-surface region and its consequent blocking of hydrogen transport. This often results in incomplete hydriding reactions and greatly impedes the kinetics in general. ${ }^{25}$ The following steps were taken to ameliorate these problems. 
Since $\mathrm{MgO}$ is a very stable oxide $(\Delta \mathrm{H} \sim 144 \mathrm{kcal} / \mathrm{mol})$, little can be accomplished for its chemical reduction by in-situ processing at low temperatures. It has generally been found that, during the so-called "activation" of these hydrides, the oxides of the as-received materials were cracked to permit hydrogen entry. This activation usually consists of somewhat abrupt, high pressure $\mathrm{H}_{2}$ cycling at elevated temperatures. In an activated sample, the oxides act as inert chaff and are of little consequence to the material inventory. Therefore, precautions were taken to minimize postactivation oxidation by employing ultra-high vacuum techniques and working in oxygen-free, allmetal systems. These will be discussed in more detail in the experimental section.

Pedersen, et al., ${ }^{25}$ observed that in $\mathrm{Mg}$ foils with a thickness of $50 \mu \mathrm{m}$ or less the kinetic problems associated with near-surface hydride precipitation was minimal. Thus, the alloy mean particle sizes in this study were limited to diameters of 100 microns or less to limit this effect. These relatively small particle sizes also had the beneficial effect of increasing the nominal active surface to volume ratio which further aided the kinetics.

The remaining problem was to improve hydrogen dissociation and recombination processes on the alloy surfaces. Nickel was our initial choice due to a number of factors: First, it is readily available and reasonably inexpensive. Second, it has a high hydrogen solubility and diffusion constant. ${ }^{26}$ Third, it readily alloys with magnesium to form a kinetically active, hydride phase $\left(\mathrm{Mg}_{2} \mathrm{Ni}\right){ }^{27}$ Fourth, there exist commercial batch processes which can be used for coating metal particles with nickel..$^{28}$

\section{Experimental}

The preparation of the materials used in the experiments consisted of several steps that varied slightly for each particular alloy to accommodate the form in which it was received. Except where noted, all of the alloys materials were reduced to powder form before study by grinding or milling the material to particle dimensions of less than $100 \mu \mathrm{m}$. Microscopic analyses were performed at all stages of these processes and the degree to which these modifications altered the alloy was ascertained by subsequent microprobe analysis.

The as-received materials came in three different forms: powders, chunks and ingot castings. Magnesium, nickel and $\mathrm{Al}_{3} \mathrm{Mg}_{2}$ came in powder form. The $\mathrm{Mg}(-325 \mathrm{mesh})$ was purchased from a commercial vendor ${ }^{29}$ as a chemically precipitated powder. The Ni (-120 mesh) was fabricated by gas atomization and generally spherical in shape. ${ }^{29}$ Professor E. Lavernia and his group at the University of California, Irvine ${ }^{30}$ prepared the gas atomized powders of $\mathrm{Al}_{3} \mathrm{Mg}_{2}(-325$ mesh, also spherical). Magnesium Elektron ${ }^{31}$ supplied samples of the various $\mathrm{AZ}$ casting alloys as well as the binary $\mathrm{Mg}_{17} \mathrm{Al}_{12}$ (cast ingots). Detailed chemical analysis was not performed to verify the quoted compositions of the suppliers, but subsequent microprobe measurements of the material before and after the hydride studies were performed. X-ray diffraction analysis also was performed on the U.C. Irvine material to verify the crystallography of the powders.

Following milling, the powdered sample materials (1-5 gms) were initially loaded into single ended containers (Figure 3 ) and lightly pressed to optimize physical contact. A deformable gasket ring $(\mathrm{Cu})$ with a stainless steel frit ( 30 micron pore size, $2 \mathrm{~mm}$ thick) was placed directly over the 
sample material. The ring provides a leak tight seal and the frit ensures containment of the powder during subsequent measurements. Grooves in the container flanges permit the insertion of sheathed thermocouples (Type $\mathrm{K}$ ) for monitoring the sample temperature. Calibration measurements were taken which showed the average temperature within the sample container to be within $\pm 2^{\circ} \mathrm{C}$ of the measured value. In this configuration, the sample container was wrapped with a strap heater and glass insulation and attached to the gas system. Leak checking was done using the system mass spectrometer following standard vacuum practice. The calibrated sensitivity of the leak checking arrangement was $10^{-11} \mathrm{std}-\mathrm{ml} / \mathrm{sec}$.

Temperatures determined in the above sample holder were very representative of the sample under equilibrium conditions. However, when the conditions deviated from equilibrium, it is doubtful that the thermocouple accurately reflected the interior temperature of the sample. Non-equilibrium conditions occurred in larger samples where the hydriding reactions progressed very rapidly. In such cases, temperature changes due to the dissipation or absorption of reaction heat through powders of low thermal conductivity are generally not well represented by a single temperature measurement. As such, detailed microscopic rate determinations were not made and the evolution of pressure versus time was used as a qualitative measure of the kinetic performance of samples of comparable size and weight.

The experimental gas handling system (Figure 4) was built to provide flexibility in resolving hydrogen behavior over a large dynamic range. Additionally, ultra-high vacuum procedures were followed to limit the introduction of impurities during the outgassing and measurement processes. Two sample stations (high pressure and low pressure) are indicated in the system schematic with the associated vacuum and gas supply systems. As depicted, there are essentially two vacuum systems that operate at nominal pressures of $10^{2} \mathrm{~Pa}$ and $10^{-7} \mathrm{~Pa}$ to cover the intermediate and ultrahigh vacuum (UHV) ranges, respectively. As mentioned above, provision is made on the UHV system for a quadrapole mass spectrometer that is capable of detecting and resolving gas species with partial pressures of $10^{-9} \mathrm{~Pa}$ in the mass range of 1-100 amu.

In a typical sample run, the material was pumped to high vacuum at room temperature, followed by heating to $50^{\circ} \mathrm{C}$. The degree of outgassing dictated the duration and level of vacuum pumping required. Once the sample dropped to $10^{-6} \mathrm{~Pa}$ at $50^{\circ} \mathrm{C}$, the temperature was increased to $150^{\circ} \mathrm{C}$ and left until the system pressure was again reduced to $10^{-6} \mathrm{~Pa}$. At this point, the sample was exposed to successive cycles of hydrogen exposure $\left(10^{4} \mathrm{~Pa}\right)$ followed by vacuum pumping until the partial pressure of water vapor was less than $10^{-7} \mathrm{~Pa}$ and the dominant gas species was hydrogen. This procedure has been found be very effective in initially cleaning hydride materials.

PCT and kinetic behavior are measured in "Sieverts" 32 manner using standard gas volumetry techniques. In this technique, the sample is exposed to hydrogen in a series of aliquots or charges. Each aliquot progressively loads the solid to higher concentrations and is terminated by the pressure coming to an equilibrium value. Desorption is performed simply in reverse. In both steps, the behavior of the pressure with time reflects the rate behavior. Both sample stations were sized so that sufficient pressure changes could be made to permit an accurate measure of the material behavior during hydrogen absorption or desorption. Volume calibrations were performed for each sample run using volume standards that are traceable to National Bureau of Standards (NBS) primary standards. Due to the small size of the vessels and the high pressures used with 
diaphragm-based pressure gauges, the manifold volumes had to be calibrated as a function of pressure over the range of measurement. The resulting least-squares fit of the pressure dependence of the volume yielded a self-consistent hydrogen gas inventory in non-absorbing calibration samples (SS 304 slugs). Thus, these volume fits were used in place of a fixed volume parameter in subsequent PCT calculations.

The high pressure PCT system is a low volume system $(\sim 4 \mathrm{ml})$ that is certified for pressures up to $20 \mathrm{MPa}$. Since the hydrogen inventory in the sample is deduced from the gas phase pressures, two pressure transducers were employed to optimize range and sensitivity. For high pressure measurements ( $>1 \mathrm{MPa})$, a quartz crystal pressure transducer is used with $0.01 \%$ accuracy and $5 \mathrm{x}$ $10^{-8}$ full scale (F.S.) resolution. For the lower pressures $(<1.3 \mathrm{MPa})$, a capacitive manometer is used with a resolution of $10 \mathrm{~Pa}$. The temperature is measured and maintained using a closed loop control system ( $<5 \%$ F.S. setpoint). For large temperature changes, the system will fully equilibrate in less than one hour.

Microscopic analyses of the sample materials before and after hydriding were made using an electron microprobe system (JEOL 733 Superprobe ${ }^{\circledR}$ ) with both wavelength and energy dispersive spectrometers. For samples that required a much finer spatial resolution of structures, a field emission scanning electron microscope (FESEM) was used with a resolution of up to 1.5 nm. (JEOL 6400 FESEM with LINK $^{\circledR}$ Energy Spectrometer). Samples were prepared metallographically; that is, potted, cross-sectioned and polished because this geometry yields good quantitative data on compositional structure. Generally, backscattered electron imaging was used because contrast in this mode is a strong function of atomic number, $\mathrm{Z}$, and, hence, different phases were clearly delineated.

\section{Results and Discussion}

\section{Kinetic Improvements}

As previously mentioned, nickel was chosen as the initial surface coating to improve the surface kinetics of the samples. However, it was found that Ni powders and coatings would convert to $\mathrm{Mg}_{2} \mathrm{Ni}$ relatively quickly $\left(<1\right.$ hour) at temperatures as low as $250^{\circ} \mathrm{C}$ in a hydrogen gas environment. This was due to a combination of favorable thermodynamic stability of $\mathrm{Mg}_{2} \mathrm{Ni}$, good mobility of $\mathrm{Mg}$ in $\mathrm{Ni}$ and the high vapor pressure of magnesium $\left(10^{4} \mathrm{~Pa}\right.$ at $\left.300^{\circ} \mathrm{C}\right) .{ }^{33}$ Thus, a more stable structure is produced with better hydrogen kinetic properties. Much of the kinetic improvements that we found may be attributed to the communication between this phase and other hydride phases forming in the bulk.

Two mechanisms were found to provide enough contact between $\mathrm{Mg}$ and $\mathrm{Ni}$ for a reaction to occur. One mechanism was simply the direct contact of $\mathrm{Ni}$ at the surface of the $\mathrm{Mg}$ alloys. The other can be attributed to the presence of $\mathrm{Mg}$ vapor which reacts with any exposed $\mathrm{Ni}$ surface within the sample space. In general, the potential for vapor transport of $\mathrm{Mg}$ to all surfaces in the sample space must be considered in the interpretation of measurements on Mg-based alloys. This effect was often not considered in previous studies. 
This vapor process can be used advantageously to fabricate magnesium alloys with improved properties. This was demonstrated by forming $\mathrm{Mg}_{2} \mathrm{Ni}$ in-situ by separately loading $\mathrm{Ni}$ and $\mathrm{Mg}$ powders and heating to $300^{\circ} \mathrm{C}$ in hydrogen. The product is very finely divided particles of $\mathrm{Mg}_{2} \mathrm{Ni}$ whose sizes were largely dictated by that of the initial $\mathrm{Ni}$ particles. In Figure 5, the $\mathrm{Mg}_{2} \mathrm{Ni}$ reaction product can be seen to be formed around the Ni cores. This sample was chosen for illustration in which the Ni core can still be seen in some of the incompletely reacted particles; in practice, the reaction is taken to completion. Also shown are the residual $\mathrm{Mg}$ fragments or hollow shells which consist of $\mathrm{MgO}$ and are the remnants of the native surface oxide originally surrounding the asreceived $\mathrm{Mg}$ powder. Microprobe analysis of the reacted $\mathrm{Mg}_{2} \mathrm{Ni}$ particles revealed them to be single phase. This is in contrast to $\mathrm{Mg}_{2} \mathrm{Ni}$ material formed by ingot metallurgy shown in Figure 6 . Since $\mathrm{Mg}_{2} \mathrm{Ni}$ is a eutectic alloy, it is not surprising to find a mixture of $\mathrm{Mg}$ and $\mathrm{Mg}_{2} \mathrm{Ni}$ phases. This material microstructure would require further material processing to achieve the homogeneity of the vapor phase reacted $\mathrm{Mg}_{2} \mathrm{Ni}$ particles. The ability to form single phase materials at such low temperatures may prove to be an advantage in hydride applications. First of all, it increases the $\mathrm{Mg}_{2} \mathrm{Ni}$ fraction of storage material. Also, since the particle size is largely dictated by the starting Ni powder size, this can to fabricate a more active material in-situ without additional handling. Finally, limited cycling studies did not indicate particle break-up or decrepitation in this vapor phase reacted material. (Continued decrepitation with hydrogen cycling is an important practical problem for applications and is often exacerbated by the multi-phase makeup of intermetallics such as $\mathrm{Mg}_{2} \mathrm{Ni}$. Additional filtering which may be needed to reduce transport and settling of fines can greatly complicate hydride bed designs.) Specific surface areas were not determined, so quantitative kinetic comparisons could not be made; however, the reacted powder had much better hydrogen kinetics than conventional powder of nominally equivalent particle size. Both are an improvement over that of $\mathrm{Mg}$ alone.

Two methods were used in our experiments to provide kinetic enhancement for hydrogen measurements on Mg-based alloys. Each started with $\mathrm{Ni}$ and resulted in the formation of other phases, such as $\mathrm{Mg}_{2} \mathrm{Ni}$, by hydrogen cycling. The first method was to simply put small, active $\mathrm{Ni}$ particles in intimate contact with the sample alloy. This was done using fine $(\sim 40 \mu \mathrm{m})$ powders of pure $\mathrm{Ni}$ and mechanically mixing it with the alloy in an impact mill for 5 minutes' duration. The goal was to provide good contact between the $\mathrm{Ni}$ and the alloy without forming bulk alloys. The milling process can cause significant mechanical alloying with longer periods of operation. ${ }^{34}$ In a few cases, the effect of milling time on hydriding and composition was systematically studied. It was found that longer milling times resulted in the formation of $\mathrm{Mg}_{2} \mathrm{Ni}$ at the interfaces of the alloy particles. The shorter milling time reduced the occurrence of this reaction during milling and had the beneficial effect of further reducing the size of alloy shavings. Grab samples of the material were taken just after milling for SEM analysis and comparison with the material after hydrogen exposure. Ni powder was added in a 1:1 ratio to the $\mathrm{Mg}$ atomic content of the alloy. This ratio was far more than needed for simply coating the $\mathrm{Mg}$ surfaces. The excess Ni was not removed prior to measurements, but did not affect the hydride measurements because of its low solubility. The distribution of Ni particles on a typical alloy is shown in the Figure 7.

The second method for $\mathrm{Ni}$ deposition used a commercial process ${ }^{26}$ which involves the decomposition of nickel carbonyl at temperatures in excess of $200^{\circ} \mathrm{C}$. The result of the decomposition is a gaseous product and a pure $\mathrm{Ni}$ precipitate which adheres to the alloy particles. Thin uniform coatings were produced that did not exhibit any impurities during outgassing and 
were easily activated. Figure 8 is a micrograph of a cross-sectioned alloy particle with the coating and shows the uniformity that could be achieved. Pure magnesium, as well as several $\mathrm{Mg}-\mathrm{Al}$ alloys were successfully treated in this way. However, the formation of $\mathrm{Mg}_{2} \mathrm{Ni}$ during subsequent hydrogen measurements destroyed the integrity of the coating/bulk interface by producing a "delaminated" shell of $\mathrm{Mg}_{2} \mathrm{Ni}$ which was physically separated from the sample (Figure 9). This rendered the coating useless as a means for transporting hydrogen to the bulk. A more detailed investigation of thickness variation and adhesion was not pursued in this study.

In general, it was found that $\mathrm{Ni}$ was required to achieve significant hydrogen uptake in $\mathrm{Mg}$-based alloys. Therefore, the addition of $\mathrm{Ni}$ became a consistent part of the following hydride results, with the few exceptions noted. All of the hydride alloy data in which $\mathrm{Ni}$ was added used the contact method because of the delamination problem mentioned above. The contact method of application proved the concept of separately forming a near-surface, kinetically active phase for these Mg-based alloys.

\section{Bulk Alloy development}

The $\mathrm{Mg}-\mathrm{Al}$ alloy phase diagram is pictured in Figure 10.35 Essentially three phases are available for detailed study. These are the $\beta$ phase $\left(\mathrm{Al}_{3} \mathrm{Mg}_{2}\right)$, the $\gamma$ phase $\left(\mathrm{Mg}_{17} \mathrm{Al}_{12}\right)$ and the dilute $\mathrm{Al}$ solubility range. The latter extends only up to compositions of approximately 10 at.\% $\mathrm{Al}$, while the other phases are line compounds with small compositional ranges at the temperatures of interest. There also exists an $\mathrm{R}$ phase in a limited temperature range $\left(300^{\circ} \mathrm{C}-350^{\circ} \mathrm{C}\right)$ between the $\beta$ and $\gamma$ phases. Controversy exists concerning the formation mechanism of the $\mathrm{R}$ phase ${ }^{36}$ and it was not chosen as a starting alloy phase. Nevertheless, its existence may have some applicability to these results and will be discussed further in the text.

\section{AZ Alloys:}

The AZ series of magnesium alloys are readily available as sand cast alloys which contain varying amounts of aluminum and zinc. In addition to the points mentioned previously, their use in this study as a starting material also eliminated the need for custom alloy fabrication. In general, the $\mathrm{Al}$ concentration in these alloys falls just short of the solubility limit of aluminum in magnesium (11 wt. $\%$ at $437^{\circ} \mathrm{C}$ ). The addition of zinc lowers the melting point slightly and significantly improves the ductility of the Mg-Al base. The nomenclature for these alloys includes the first letter of the major additives to magnesium followed by their respective weight percentages. For example, AZ31 is nominally $96 \mathrm{wt} . \% \mathrm{Mg}, 3 \mathrm{wt} . \% \mathrm{Al}$ and $1 \mathrm{wt} . \% \mathrm{Zn}$. The $\mathrm{AZ} \mathrm{31,AZ} 61$ and $\mathrm{AZ} \mathrm{91,}$ alloys were chosen to study the effect of the $\mathrm{Al}$ content while keeping the $\mathrm{Zn}$ constant. The compositional limits of the three alloys studied are shown in Table I. Also, these alloys are very close to the solubility limit of $\mathrm{Al}$ in $\mathrm{Mg}$ at these experimental temperatures $\left(\sim 300^{\circ} \mathrm{C}\right)$. Therefore, they provide a means to explore the effect of the onset of the $\gamma$ phase on hydride performance.

\section{TABLE I. COMPOSITIONAL LIMITS OF AZ ALLOYS}

$\begin{array}{llllll}\text { alloy } & \text { Al wt.\% } & \text { Zn wt.\% } & \text { Mn wt.\% } & \text { Ni wt.\% } & \text { Cu wt.\% } \\ \text { AZ31 } & 2.5-3.5 & 0.6-1.4 & 0.2 \mathrm{~min} . & 0.005 & 0.05 \\ \text { AZ61 } & 5.8-7.2 & 0.4-1.5 & 0.15 \mathrm{~min} . & 0.005 & 0.05 \\ \text { AZ91 } & 8.3-9.7 & 0.35-1.0 & 0.13 \mathrm{~min} . & 0.03 & 0.1\end{array}$


In their natural form, the alloys were practically inert to hydrogen absorption and Ni powder was required to improve the kinetics as previously described. Following the outgassing and pretreatment steps described earlier, activation was initiated by increasing the hydrogen pressure to $15 \mathrm{MPa}$ and the temperature to $\sim 330^{\circ} \mathrm{C}$. After the initial step, the hydrogen was immediately outgassed or desorbed. This cycle was repeated a number of times to provide good uptake kinetics and reproducible PCT measurements. Following this treatment, the samples generally behaved reproducibly; however, in some cases a further evolution in behavior was reflected in the data as the number of PCT cycles were increased. This is noted in the specific instances.

$\boldsymbol{A Z}$-31-SEM and Energy Dispersive X-ray (EDX) analysis of the as-milled AZ 31 material suggested very little evidence of mechanical alloying. The particles were analyzed in cross-section and, as shown in Figure 11, are 100-200 $\mu \mathrm{m}$ across and $30 \mu \mathrm{m}$ thick chunks of alloy intermixed with the nickel particles. Many of the nickel particles adhered to the larger alloy chunks due to the mechanical action of the milling process. EDX revealed the unhydrided alloy material to be slightly richer in magnesium ( $-97-99 \mathrm{wt} . \%)$ and less than the $\mathrm{Al}(1-2 \mathrm{wt} . \%)$ given in the nominal specification. $\mathrm{Zn}$ and $\mathrm{Mn}$ were observed in compositions of less than $0.5 \mathrm{wt} \%$. Also, second phases were observed which are much richer in aluminum and determined to be $\mathrm{Mg}_{17} \mathrm{Al}_{12}$. The nickel particles were observed to be pure and roughly spherical in shape.

After hydrogen cycling, an alloying reaction was found to have occurred between $\mathrm{Mg}$ and $\mathrm{Ni}$ on the $\mathrm{Ni}$ particles. Compositional analysis was consistent with the formation of $\mathrm{Mg}_{2} \mathrm{Ni}$. This can be observed in Figure 12, where after approximately 200 hours of hydrogen cycling above $300^{\circ} \mathrm{C}$, most of the nickel particles were completely reacted. The formerly smooth surface of the $\mathrm{Ni}$ particles are now deformed due to the reaction and subsequent increase in size. The diameter of the largest completely reacted $\mathrm{Ni}$ particle was found to be approximately $40 \mu \mathrm{m}$. Larger particles still retain an unreacted $\mathrm{Ni}$ core. It should also be noted that the $\mathrm{Mg}_{2} \mathrm{Ni}$ regions also contained up to 2-3 wt.\% $\mathrm{Zn}$. Although there is a strong possibility of $\mathrm{Zn}$ vapor transport ( $\mathrm{Zn}$ has a higher vapor pressure than $\mathrm{Mg}$ ), similar transport of a $\mathrm{Mg}-\mathrm{Zn}$ alloy cannot be ruled out. In the alloy particles, the $\mathrm{Mg}$ fractional composition dropped to $94-97 \mathrm{wt} . \%$ while the relative $\mathrm{Al}$ composition increased to $\sim 3 \mathrm{wt} . \%$. Among the other elements in the original alloy particles, the $\mathrm{Mn}$ fraction remained constant while the $\mathrm{Zn}$ decreased by an order of magnitude. The formation of $\mathrm{Mg}_{2} \mathrm{Ni}$ appeared as the dominant, final constituent for most of these experiments.

A portion of the as-milled alloy with $\mathrm{Ni}$ was subsequently melted in a differential scanning calorimeter to determine the thermal behavior without hydrogen. The background gas was argon. The melting temperature was determined to be $630^{\circ} \mathrm{C}$ which is consistent with published data for AZ31 alloys. ${ }^{36}$ Cooling indicated a solidification reaction at $500^{\circ} \mathrm{C}$ which would indicate the formation of $\mathrm{Mg}_{2} \mathrm{Ni}$ according to published phase diagrams. ${ }^{35}$ These measurements verified the AZ31 thermal behavior and suggested that if liquid alloy were available, the $\mathrm{Mg}_{2} \mathrm{Ni}$ reaction would proceed very quickly. Since the experimental conditions did not approach the melt, this reaction must have been the result of significant $\mathrm{Mg}$ vapor transport.

The equilibrium hydrogen pressure and temperature behavior of the AZ 31 alloy with the addition of $\mathrm{Ni}$ was very similar to the that of $\mathrm{Mg}_{2} \mathrm{NiH}_{4}$. At $300^{\circ} \mathrm{C}$, most of the Al would be expected to be in solution and any effects should be reflected in the PCT values. In practice, the material readily 
activated after only three activation cycles. PCT measurements were made on the fourth hydrogen cycle and Figure 13 is a plot showing the pressure behavior with time during the three loading aliquots. It reflects the typical loading behavior with time. Over half $(0.59 \mathrm{H} / \mathrm{Mg})$ of the hydrogen was loaded in about one minute, while the time required to absorb the remainder of the gas was significantly greater. This was found to be typical loading behavior for this alloy as well as upon desorption. In Figure 14, this is shown by the fall off in the desorption rate at an $\mathrm{H} / \mathrm{Mg}$ ratio of approximately 0.6 at $300^{\circ} \mathrm{C}$. The numbers on the plot indicate the sequence in which the aliquots of gas were removed as the concentration was decreased across the plateau. Thus, after the fourth aliquot, the rate is slowing as an $\mathrm{H} / \mathrm{Mg}$ value of 0.6 is approached. During desorption at $\mathrm{H} / \mathrm{Mg}$ less than 0.6 , the sample required re-heating to $330^{\circ} \mathrm{C}$ and active vacuum pumping to remove the remaining hydrogen. This residual hydrogen inventory was suggestive of $\mathrm{MgH}_{2}$ and this lower plateau was not explored in detailed due to very slow kinetics. The PCT plateau is shown in Figure 15 and extends from an $\mathrm{H} / \mathrm{Mg}$ ratio of 0.6 up to about 1.0. The hydrogen concentration below $0.6 \mathrm{H} / \mathrm{Mg}$ is due to $\mathrm{MgH}_{2}$, as mentioned. Several additional points should be observed from Figure 15. Above $0.6 \mathrm{H} / \mathrm{Mg}$, the plateau is both flat and very close in values that might be expected of $\mathrm{Mg}_{2} \mathrm{Ni}$. Additionally, the limiting ratio of $1.0 \mathrm{H} / \mathrm{Mg}$ would suggest that only half of the total $\mathrm{Mg}$ in the sample is participating in the hydride phase (if a ratio of $2.0 \mathrm{H} / \mathrm{Mg}$ is taken as the stoichiometric value for both $\mathrm{Mg}$ and $\mathrm{Mg}_{2} \mathrm{Ni}$ ). Of this half of the magnesium, approximately $40 \%$ reacted with the $\mathrm{Ni}$ particles to form $\mathrm{Mg}_{2} \mathrm{Ni}$. As the number of cycles increased, more $\mathrm{Mg}$ reacted and the $\mathrm{H} / \mathrm{Mg}$ ratio approached 2.0. However, the width of the plateau attributed to $\mathrm{Mg}_{2} \mathrm{Ni}$ remained nearly constant indicating little further reaction. The measurements were consistent with the SEM analysis.

The AZ 31 sample did show significant hydrogen capacity and that kinetic activation could be effectively achieved. However, the results suggest that small additions of Al do not substantially affect the stability of the $\mathrm{Mg}$ hydride phases. After hydriding, the existence of $\mathrm{Zn}$ within the matrix phase was not observed and, therefore, the effects of $\mathrm{Zn}$ on the hydride could not be ascertained.

$A Z 61$-The AZ61 alloy ingot was also turned by lathe and then mechanically milled with Ni for nominally five minutes. In Figure 16, the sample condition can be observed prior to hydrogen exposure. The appearance of the alloy chunks are very similar to AZ31 with the surrounding $\mathrm{Ni}$ particles being for the most part separate. Analysis of the AZ-61 alloy material after the mechanical milling reveals the existence of second phases composed of magnesium, aluminum and an enriched $\mathrm{Zn}$ composition. This $\mathrm{Zn}$ enriched, phase with much smaller concentrations in the matrix, accounts for the nominal $1 \mathrm{wt} \% \mathrm{Zn}$ composition of the alloy. The background matrix composition was found to be similar to AZ31 and AZ 91. As will be discussed below, two characteristic morphologies resulted from hydriding: a highly reacted alloy with excellent hydride behavior; and one with behavior not too different from AZ31 above. Most of the following micrographs and discussion involve the more highly reacted material.

Upon hydriding, one can see in the four frames of Figure 17 that the second phase components in the starting alloys are missing and there is greater microstructural complexity. If one examines in detail the area in the fourth frame (Figure 18), it becomes apparent that the internal Zn-bearing phases have vanished, leaving voids behind and perhaps reacting with the $\mathrm{Ni}$ particles directly adjacent to the alloy particles. The relative $\mathrm{Mg}$-Al composition has also changed within the alloy 
particles with an increase in the aluminum fraction of the matrix. A second phase, $\mathrm{Mg}_{17} \mathrm{Al}_{12}$, has increased in volume within the matrix as well. Using detailed X-ray analysis with high resolution FESEM, it was found that each alloy particle had an $\mathrm{Al}$ composition gradient from the interior to the surface. In the core, the Al content was only slightly higher than the original matrix, but a zone of reduced $\mathrm{Al}$ concentration appeared nearer to the surface. In Figure 18, this coincided with a reduction in the $\mathrm{Mg}_{17} \mathrm{Al}_{12}$ phase near the boundaries. In areas where a reaction zone abutted the particle, the $\mathrm{Al}$ content was much lower, with no $\mathrm{Mg}_{17} \mathrm{Al}_{12}$ observed in the near-surface region. In areas where the second phase precipitates of $\mathrm{Mg}_{17} \mathrm{Al}_{12}$ intercept the boundary, no $\mathrm{Ni}$ is observed. This is seen in the lower right of the Figure 18. Further high resolution analysis of the reaction zones (Figure 19) revealed two regions that are further magnified in Figures 20 and 21 . In Figure 20 , solid phases of $\mathrm{Ni}_{3} \mathrm{Al}$ are observed as extended ridges where the Ni interface would have been. In Figure 21, a grainy texture can be observed near the surface of the alloy particle. These regions were found to be a mixture of $\mathrm{Mg}$-rich and $\mathrm{Ni}_{3} \mathrm{Al}$ phases. The extremely small size $(<10 \mathrm{~nm})$ of the Mg phases did not permit further identification of species other than $\mathrm{Mg}$. Thus, regions of markedly different phases are found across each alloy particle. This was a distinguishing characteristic of the sample that hydrided favorably.

Microscopy on all AZ61 samples showed little discernible difference prior to the hydride measurements. However, in samples that did not exhibit favorable hydride behavior, the complex transformations were not apparent and the reaction zones (Figures 20,21) did not form. This can be seen in the sample shown in Figure 22. Residual hydrogen was left on this sample by terminating the dehydriding process after the $\mathrm{Mg}_{2} \mathrm{NiH}_{4}$ - like behavior, but before the $\mathrm{MgH}_{2}$ behavior was fully desorbed. The hydride phase can be observed well into the bulk but not within the second phase regions. (The darker hydride contrast would disappear when the beam was focused on a hydride region due to desorption of the hydrogen.) Also, the $\mathrm{Zn}$ rich second phases were present in subsequent AZ61 samples after hydriding and may play a role in forming the reaction zones in the better material. In the non-reacted samples (Figure 22), the formation of $\mathrm{Mg}_{2} \mathrm{Ni}$ on the Ni particles was the dominant effect observed.

Results from scanning calorimetry on the AZ61 material as mixed with $\mathrm{Ni}$ did not indicate any additional reactions on the first scan. Later scans always indicated the formation of $\mathrm{Mg}_{2} \mathrm{Ni}$ as in other $\mathrm{AZ}$ alloys. Repeated scans on several samples without the appearance of any additional phases suggests that high pressure hydrogen may be necessary for other transformations to occur.

Prior to PCT measurements, the sample material with the reaction zones was activated three times to a pressure of $15 \mathrm{MPa}$ at $\sim 330^{\circ} \mathrm{C}$. The PCT measurements showed a behavior that was similar to that of AZ31 through the first four cycles, that is, a high plateau resembling that of $\mathrm{Mg}_{2} \mathrm{Ni}$ evolved with non-exceptional equilibrium pressure behavior. However, during the fourth cycle the rates of hydrogen absorption and desorption increased dramatically. The desorption isotherms for the fourth and several subsequent runs are shown in Figure 23. The fourth isotherm at $325^{\circ} \mathrm{C}$ can be seen to be very similar to $\mathrm{Mg}_{2} \mathrm{Ni}$ (\#4, Figure 23). Since the kinetics were fast, the temperature was dropped to $260^{\circ} \mathrm{C}$ for the following PCT cycle (\#5, Figure 23). Unexpectedly, the desorption plateau pressure increased to a value slightly above the previous $325^{\circ} \mathrm{C}$ cycle value and exhibited very fast kinetics. Apparently, the material had transformed during this time and retained the high pressure behavior despite subsequent loading excursions to $325^{\circ} \mathrm{C}$. Some of the $\mathrm{Mg}_{2} \mathrm{Ni}$ behavior was evidenced by a second lower pressure plateau at lower concentrations on run $\# 5$. A $300^{\circ} \mathrm{C}$ 
plateau taken on the ninth cycle is shown in Figure 23 to illustrate the difference in later cycles where the lower plateau is less in evidence. One can see from the pressure-time behavior in Figure 24 that the loading kinetics of the fifth cycle at $260^{\circ} \mathrm{C}$ was faster than the fourth cycle at $325^{\circ} \mathrm{C}$. Both were exceptionally fast relative to the absorption behavior of $\mathrm{Mg}$ and $\mathrm{Mg}_{2} \mathrm{Ni}$ and this was also true for desorption. Figure 25 includes a plot of the pressure-time behavior during desorption for several aliquots of the fourth and fifth cycles. The first aliquots at both temperatures equilibrated rapidly, but later aliquots were slower. By the second aliquot at $325^{\circ} \mathrm{C}$, the sample was behaving more like $\mathrm{Mg}_{2} \mathrm{Ni}$; however, at $260^{\circ} \mathrm{C}$, the sample was still equilibrating rapidly on the fourth aliquot. Of additional note is the multi-component aspect of the initial desorption aliquot on the first aliquot at $260^{\circ} \mathrm{C}$. This behavior was not present in subsequent aliquots at the lower temperatures and may relate to the role played by hydrogen in the material transformation.

Figure 26 illustrates the reproducibility of measurements after the transformation of the material. Here the temperature was altered from $155^{\circ} \mathrm{C}$ up to $260^{\circ} \mathrm{C}$ during cycle nine to confirm that no further changes had occurred and the material was stable. Also, the reappearance of the low pressure plateau during cycle eight at $200^{\circ} \mathrm{C}$ indicated that a lower pressure phase was present in the sample although not apparent at higher temperatures. Three isotherms that best represent the final behavior of this material are illustrated in Figure 27 and include several values collected at $155^{\circ} \mathrm{C}$. At this lower temperature the kinetics were very slow. After the ninth cycle, the sample was cooled, removed from the system and carefully examined via SEM and EDX as described earlier.

Subsequent PCT measurements having similar high pressure plateaus were obtained with other alloy compositions; however, none had a combination of high pressure and kinetics comparable to this sample. The behavior in this sample correlated with two observations in the SEM results: the existence of reaction zones and the absence of $\mathrm{Zn}$-rich phases in the bulk of the alloy particles. The very fine structure of the reaction zone may be responsible for the very rapid kinetics at both the surface and the bulk. In the reaction zone and throughout the bulk of the particles there is evidence for a non-equilibrium reaction to have occurred. This evidence ranges from melting of the second phase particles in certain regions to the existence of compositional gradients throughout the bulk. Four additional samples were cut from the ingot of this material, but duplication of the conditions for initiating the transformation was not achieved. Thus, although equilibrium phases are identifiable in the post-hydrided material, the role of hydrogen could not be determined and the existence of metastable phases cannot be ruled out.

AZ91-The AZ 91 alloy displayed distinctly different mechanical properties during the turning process and resulted in larger ( $400-500$ micron) starting fragments than the other alloys. SEM micrographs (Figure 28) revealed the matrix and second phases typically seen in these alloys. The nominal composition was again found to be slightly low in $\mathrm{Al}$ with a consequent larger fraction of $\mathrm{Mg}$. The $\mathrm{Al}-\mathrm{Zn}$ phases differed slightly in composition due to additional concentrations of $\mathrm{Mn}$. Figure 29 shows the effects of hydrogen cycling, where the $\mathrm{Mg}-\mathrm{Ni}$ reaction zones formed around the Ni particles; however, EDX revealed $\mathrm{Mg}$ and $\mathrm{Ni}$ to be more in a ratio of 1:1 in the near-surface of the Ni particles (Figure 29). Also, large regions (Figure 29) of the alloy particles were $\mathrm{Mg}_{17} \mathrm{Al}_{12}$. The predominant composition of the alloy matrix was reduced in $\mathrm{Mg}$ and increased in $\mathrm{Al}$ to $92 \mathrm{wt} . \%$ and $7 \mathrm{wt} . \%$, respectively (Figure 29). The largest totally reacted Ni particle was approximately $14 \mu \mathrm{m}$ diameter with a total hydrogen exposure time of approximately 170 hours at 
temperatures greater than $300^{\circ} \mathrm{C}$. This particular micrograph shows an agglomeration of the reacted $\mathrm{Ni}$ particles, (Figure 29) which was not previously observed. Also, there was some evidence of oxidation and cracking (Figure 29) which was attributed to a very small air leak detected during the final part of this experiment.

Differential Scanning Calorimeter (DSC) thermal analysis revealed a small amount of melting occurring at $455^{\circ} \mathrm{C}$, with most of the sample melting at $590^{\circ} \mathrm{C}$. Subsequent cooling indicated the existence of solidifying phases at $580^{\circ} \mathrm{C}, 500^{\circ} \mathrm{C}$ and $420^{\circ} \mathrm{C}$. In reference to the published alloy information and the phase diagram, these temperatures would correspond to the alloy, $\mathrm{Mg}_{2} \mathrm{Ni}$ and $\mathrm{Mg}_{17} \mathrm{Al}_{12}$, respectively. ${ }^{35}$ Due to additional heats of formation of the $\mathrm{Mg}_{2} \mathrm{Ni}$ and hydriding, the local temperature during hydriding may have exceeded these melting temperatures. However, no evidence of melting was observed in samples after hydriding.

The AZ91 material required a larger number of activation cycles (five) before PCT data collection became acceptable. However, like other $\mathrm{AZ}$ alloys with the added $\mathrm{Ni}$, pressure, temperature and kinetics were similar to $\mathrm{Mg}_{2} \mathrm{Ni}$, as seen in Figure 30 . Figure 30 shows the $300^{\circ} \mathrm{C}$ isotherms of two different cycles with an excursion to $250^{\circ} \mathrm{C}$ during the seventh cycle to test material stability. The resultant desorption pressure plateau at $300^{\circ} \mathrm{C}$ was slightly higher than reported for $\mathrm{Mg}_{2} \mathrm{Ni}$. There also seemed to be a large fraction of the magnesium participating in both the overall hydrogen uptake and the $\mathrm{Mg}_{2} \mathrm{Ni}$ plateau compared to the other alloys. This is in spite of having less hydrogen exposure time at elevated temperatures and of having less reacted $\mathrm{Ni}$ (see text below). Despite much pre-cycling, the $\mathrm{Mg}_{2} \mathrm{Ni}$ phase continued to evolve, as evidenced in the increase in absorbed hydrogen from cycle seven to cycle eight. The desorption behavior is reflected in the pressure equilibration time for three aliquots in Figure 31 . The relative difference between the $250^{\circ} \mathrm{C}$ and $300^{\circ} \mathrm{C}$ temperatures are plotted and show a relatively weak temperature dependence on rate. In general, the time for equilibrating $\mathrm{Mg}_{2} \mathrm{Ni}$ is protracted at $250^{\circ} \mathrm{C}$. The difference in desorption kinetics at $300^{\circ} \mathrm{C}$ between nearly fully loaded material (third aliquot) and at lower hydrogen content (fifth aliquot).

As might be predicted from the phase diagram, as-received AZ 91 had more initial second phase $\left(\mathrm{Mg}_{17} \mathrm{Al}_{12}-\gamma\right.$ phase) than the other $\mathrm{AZ}$ alloys. The dispersion of the phase seemed uniform and, to a large extent, was not altered by the hydrogen cycling or hydride formation. The starting material also had decidedly different mechanical properties which prevented the Ni from sticking to these particles. This may account for the greater difficulty in activating the sample and its subsequent slow kinetics. It may be recalled from the AZ 61 sample results that the appearance of the $\gamma$ phase near the surface signaled the absence of a reaction zone. In AZ91 the relative abundance of the $\gamma$ phase and the reduction in $\mathrm{Ni}$ at the boundary may have contributed to a reduction in the reaction of the matrix. In this case, the eutectic matrix was activated but simply behaved more like $\mathrm{Mg}$.

\section{Mg-Al Line Compounds:}

The line compounds of the $\mathrm{Mg}-\mathrm{Al}$ system have melting points of approximately $450^{\circ} \mathrm{C}$ and range in aluminum composition from 38-60 at.\%. Three different compounds are observed within this compositional range, as depicted in the phase diagram in Figure 10. The $\gamma$ phase $\left(\mathrm{Mg}_{17} \mathrm{Al}_{12}\right)$ extends from $45-60$ at. $\%$ at $450^{\circ} \mathrm{C}$, but narrows to $52-60$ at. $\%$ at $300^{\circ} \mathrm{C}$. We often observed $\mathrm{Mg}_{17} \mathrm{Al}_{12}$ as a second phase precipitate in the $\mathrm{AZ}$ alloys. Above $370^{\circ} \mathrm{C}$ and below $320^{\circ} \mathrm{C}$, the $\gamma$ 
phase forms a mixed region with the $\beta$ phase $\left(\mathrm{Al}_{3} \mathrm{Mg}_{2}\right)$. The $\beta$ phase exists in a narrow compositional range from $38.5-40.3$ at.\%, with little or no temperature dependence. The third phase is the $R$ phase, which has very narrow compositional range of around $42 \pm 0.3$ at.\%. Between $320^{\circ} \mathrm{C}$ and $350^{\circ} \mathrm{C}$, the $\gamma, \beta$ and $\mathrm{R}$ phases exist as a mixture. Samples were obtained from both Magnesium Elektron and U. C. Irvine with nominal compositions in the $\gamma$ and $\beta$ phases and hydrogen PCT measurements were made.

$M g_{17} A l_{12}$-The $\gamma$ phase is a brittle compound that is fabricated by melting the components within the compositional limits. It was received as large chunks $(2-5 \mathrm{~cm})$, and due to the brittle nature of the material, was crushed into pieces nominally less than $1 \mathrm{~mm}$. At low temperatures, this material showed little or no hydrogen absorption behavior. To improve the kinetics, Ni was added in a 1:1 ratio to the $\mathrm{Mg}$ alloy composition like the $\mathrm{AZ}$ alloys. Later, $\mathrm{Zn}$ was also successfully employed. In either case, the alloy material was loosely mixed with $\mathrm{Ni}$ or $\mathrm{Zn}$ and impact milled to further reduce the size and mix the components. Mixing time was kept at five minutes.

The initial sample of this material with Ni proved to be relatively inert to hydrogen absorption. In an effort to initiate absorption, it was taken to $460^{\circ} \mathrm{C}$ prior to subsequent measurements. Although absorption was effectively increased, the sample did not show substantial desorption behavior at temperatures below $330^{\circ} \mathrm{C}$ on three different sample runs. This was consistent with observed reactions in the $\mathrm{AZ}$ alloys where the second phase, $\mathrm{Mg}_{17} \mathrm{Al}_{12}$ did not seem to participate in hydriding nor in reactions. In some cases, hydride phases were observed to form around these phases (see for example Figure 22).

The effects of adding $\mathrm{Zn}$ and not $\mathrm{Ni}$ were also studied. $\mathrm{Zn}$ was added to the $\mathrm{Mg}_{17} \mathrm{Al}_{12}$ by milling. the particles with $\mathrm{Zn}$ powder $(-325 \mathrm{mesh})$. This had a dramatic effect in increasing the amount of hydriding and permitted measurements to be made at lower temperatures. Figure 32 shows one complete desorption isotherm at $300^{\circ} \mathrm{C}$ and a second run made by changing the temperature during the run. The reproducibility of these points were checked by comparing the $300^{\circ} \mathrm{C}$ data point to the previous complete isotherm. Good agreement can be observed. Although the $\mathrm{Zn}$ addition permitted hydrogen desorption, the overall kinetics were limited. The benefits of the $\mathrm{Zn}$ can be seen in the equilibration times of the early aliquots of the first two cycles shown in Figure 33. It should be mentioned that there is an almost complete lack of absorption for a non- $\mathrm{Zn}$ alloy sample. Two features are of additional interest in this plot. First, there is a delay in onset of the absorption behavior of the first cycle. Secondly, there is a large increase in rate during the second cycle. Figure 34 depicts the equilibration time during desorption at three different temperatures. The addition of zinc in this sample, with its significant effect on the bulk hydriding of this alloy, appears different than the aluminum solution phase ( $\mathrm{Al} 0-10 \%$ ). In $\mathrm{Mg}_{17} \mathrm{Al}_{12}$, the $\mathrm{Zn}$ additions had a large effect on the plateau pressure.

$\mathrm{Al}_{3} \mathrm{Mg}_{2}$ - As mentioned above, the $\beta$ phase $\left(\mathrm{Al}_{3} \mathrm{Mg}_{2}\right)$ was manufactured in powder form by the gas atomization technique using nitrogen gas. This method of fabrication was chosen because the alloy was not generally available and prior experience had demonstrated that this narrow composition range could be successfully attained using a rapid solidification technique. The process yielded fairly uniform spherical particles with diameters less than $100 \mu$ (Figure 35). The figure also shows that some internal voids are present in the material. No other phases were observed and the as-received material required little outgassing. However, it did not prove to be a 
very active material for hydrogen absorption in its as-received condition and, as in the $\gamma$ phase samples, required mixing with $\mathrm{Ni}$ or $\mathrm{Zn}$.

$\mathrm{Ni}$ powder was again added in a ratio of 1:1 with the $\mathrm{Mg}$ atomic fraction and mixing was done in an impact mill for five minutes. Side reactions (in particular $\mathrm{Mg}_{2} \mathrm{Ni}$ ) involving the free $\mathrm{Ni}$, was expected to be particularly severe in $\mathrm{Al}_{3} \mathrm{Mg}_{2}$ due to its lower stability compared to $\mathrm{Mg}_{2} \mathrm{Ni}$. In order to minimize the effects of $\mathrm{Ni}$ reactions, temperatures were kept below $300^{\circ} \mathrm{C}$ during the desorption measurements. Unfortunately, the lower temperatures resulted in slow hydrogen kinetics and the collection of full PCT isotherms was sacrificed in order to probe these lower temperatures. The following procedure was followed for this alloy: the sample was loaded quickly at $300^{\circ} \mathrm{C}$ and the temperature decreased; the first desorption aliquots were removed at the lower temperature until the plateau was reached; and once a plateau was achieved, the temperature was increased and a new equilibrium point was established. In this fashion, the equilibrium pressure space was probed by temperature scans and the time at elevated temperatures was minimized. A summary van't Hoff plot was made from this data and is shown in Figure 36.

Similar to the Ni case, $\mathrm{Zn}$ was mixed with this alloy, but the amount of $\mathrm{Zn}$ used was much less $(0.5$ at.\%). Since an alloying reaction was desired, the $\mathrm{Zn}$ powder was milled for 120 minutes. Although not as effective as $\mathrm{Ni}$, the addition of $\mathrm{Zn}$ again permitted hydrogen absorption to occur as in the $\mathrm{Mg}_{17} \mathrm{Al}_{12}$ alloy. As above for $\mathrm{Ni}$, full PCT isotherms were not collected, but the temperature scanning procedure was followed. The pressure data is also plotted in Figure 36.

In general, adding $\mathrm{Ni}$ seemed to yield the fastest kinetics, while adding $\mathrm{Zn}$ resulted in higher plateau pressures. An additional sample was prepared with both additives, and comparisons of the three conditions are shown in Figures 37 and 38. In Figure 37, the endpoints are very close to the equilibrium pressure values and a comparison of the effects of the additives can be made. It reflects the faster loading kinetics of the $\mathrm{Ni}$ and the interim value reached by the $\mathrm{Ni}-\mathrm{Zn}$ mixture in both pressure and kinetics. However, upon desorption (Figure 38), the mixture more nearly approximated the $\mathrm{Zn}$ behavior indicating that the $\mathrm{Zn}$-modified hydride phase limits the desorption behavior. This is in contrast to loading, where the Ni-modified constituent dominates the kinetic processes.

\section{Summary}

The initial objectives of this study were to look for evidence of an alternative approach towards developing these Mg-based hydride alloys. In this regard, two questions may be asked: First, are these alloys capable of evolving and sustaining a stable surface phase that can contribute to good kinetics and communicate effectively with the bulk alloy? Second, can low enough concentrations of other elements be added to $\mathrm{Mg}$ to affect the stability of the hydride while not contributing significantly to the weight?

In regard to the first objective, the formation of $\mathrm{Mg}_{2} \mathrm{Ni}$ through the mixing of $\mathrm{Ni}$ definitely formed a stable surface phase that contributed to better kinetics. This was evidenced in all of the alloys and often at temperatures much below those that had been previously published in the literature. Despite the delamination of the contiguous Ni surfaces coatings, the phases that formed on the 
alloy particles where Ni was in contact were robust and did not degrade with limited cycling. This suggests that a coating procedure could be optimized to prevent delamination and a larger active surface phase could be nucleated in a more controlled fashion. Perhaps more importantly, the reaction zones of the AZ61 sample showed the existence of an extremely effective surface phase. Whether this increased activity is a product of the observed complex microstructure or the $\mathrm{Ni}_{3} \mathrm{Al}$ reaction, or both, requires further investigation. Clearly, the intentional formation of surface phases in $\mathrm{Mg}$ alloys is a tractable approach to improving the kinetics of surface processes.

In regard to the second question, small additions were attempted with both $\mathrm{Al}$ and $\mathrm{Zn}$. Small concentrations of $\mathrm{Al}$ seem to have little effect on $\mathrm{Mg}-\mathrm{H}$ behavior, as was evidenced by the $\mathrm{AZ}$ alloys and previous results. However, higher concentrations of Al were found to improve the pressure-temperature properties. The results presented here on the AZ 61 alloy and the earlier work of Reilly and Wiswall with 0.55 at.\% Al (Figure 39), indicate a significant reduction in stability with some sacrifice in weight. As seen in the $A Z 61$ sample, the high plateau pressures can extend the $\mathrm{Mg}$ alloys to temperatures less than $200^{\circ} \mathrm{C}$. Temperatures in this range offer much greater applicability in terms of increased system efficiency and material requirements.

$\mathrm{Zn}$ was also demonstrated as a minor additive for modifying the $\mathrm{Mg}-\mathrm{H}$ stability. The mechanism for this alteration needs further work; however, the very small concentrations that we used to change the PCT behavior suggest that some of the rationale for the use of $\mathrm{Zn}$ in structural alloys may also carry over into hydride performance. Additionally, this work also points to the difference that can exist between modifications of loading behavior versus those of desorption. Modifications which increase the loading kinetics may have little practical value if they do not affect the desorption rate limiting processes.

Finally, the high vapor pressure of $\mathrm{Mg}$ is a property which must be considered in the fabrication of alloys and yet has been largely ignored in $\mathrm{Mg}$ hydride alloy studies. The relative ease with which interdiffusional processes occur in $\mathrm{Mg}$ alloys suggest that it cannot be ignored as an agent in surface reactions, especially in the presence of hydrogen. This is often not addressed in surface studies where the effective Mg pumping speed can be quite high. In hydride reaction beds, the surface to volume ratio is much higher and the effects are more pronounced. This was demonstrated to be an advantage in forming near-surface $\mathrm{Mg}$ alloy phases and in fabricating $\mathrm{Mg}$ alloys. For the fabrication of $\mathrm{Mg}_{2} \mathrm{Ni}$ in particular, this method was demonstrated to have considerable merit in the formation of a single phase alloy. As such, it provides a more suitable hydride alloy material several ways: First, as a single phase alloy, it provides a more efficient use of reactants. Second, preliminary results suggest that it is less prone to decrepitation and particle break-up. Third, it permits in-situ alloy formation which eliminates post-fabrication handling. These factors are particularly important for the practical application of Mg-based hydrogen storage alloys. 


\section{References}

1. H. Buchner, Hydrogen Energy Systems, in Proceedings of the International Symposium on Hydrides for Energy Storage, Geilo edited by A. F. Anderson and A. J. Maeland (Pergamon Press, Oxford, 1977), p. 569.

2. C. Carpetis, Storage, Transport and Distribution of Hydrogen, in Hydrogen as an Energy Carrier, edited by C. J. Winter and J. Nitsch (Springer-Verlag, Berlin, Heidelberg, 1988), p. 249.

3. M. A. DeLuchi, Int. J. of Hydrogen Energy, 14:81 (1989).

4. G. Alefield and J. Volkl, eds., Hydrides in Metal I. \& II. (Springer, Berlin-Heidelberg,1978).

5. L. Schlapbach, ed. , Hydrides in Intermetallic Compounds I. \& II. , (Springer, BerlinHeidelberg, 1988).

6. R. G. Barnes, ed., Hydrogen Storage Materials, (Trans-Tech, Switzerland-Germany-UKUSA, 1988).

7. T. B. Flanagan, Thermodynamics of Metal Alloys and Intermetallic/Hydrogen Systems in Proceedings of the International Symposium on Hydrides for Energy Storage. Geilo edited by A. F. Anderson and A. J. Maeland (Pergamon Press, Oxford, 1977), p. 43.

8. A. R. Miedema, K. H. J. Bucshow, H. H. van Mal, J. of Less Commion Met., 49:463 (1976).

9 P. C. P. Bouton and A. R. Miedema, J. of Less Common Met., 71:147 (1980).

10. A. L. Shilov, M. E. Kost and N. T. Kuznetsov, J. of Less Common Met., 105:221 (1985).

11. R. Griessen, Phys. Rev. B., 38 6:3690 (1988).

12. C. E. Lundin, F. E. Lynch and C. B. Magee, J. of Less-Common Met., 56:19 (1977).

13. B. K. Rao, P. Jena, Phys. Rev. B., 31:6726 (1985).

14. K. Yvon, J. Schefer, F. Stucki, Inorg. Chem., 20:2776 (1981).

15. D. L. Douglas, The Storage and Release of Hydrogen from Magnesium Alloy Hydrides for Vehicular Applications, in Proceedings of the International Symposium on Hydrides for Energy Storage, Geilo edited by A. F. Anderson and A. J. Maeland (Pergamon Press, Oxford, 1977), p. 151. 
16. J. F. Nachman and D. A. Rohy, Magnesium-Alloy Hydrides in Proceedings of the International Symposium on Metal-Hydrogen Systems, Miami edited by T. N. Veziroglu (Pergamon Press, New York, 1982) p. 557.

17. P. Selvam, B. Visanathan, C. S. Swamy and V. Srinivasan, Int. J. Hydrogen Energy, 11:169 (1986).

18. J. J. Reilly, R. H. Wiswall, K. C. Hoffman and C. H. Waide, Metal Hydrides as Hydrogen Storage Media in Proceedings of Seventh Alternate Power Systems DivisionContractors Coordination Meeting (Ann Arbor MI, May 1973).

19. M. H. Mintz, Z. Gavra, G. Kimmel and Z Hadari, J. of Less-Common Met., 74:263 (1980).

20. F. G. Eisenberg, D. A. Zagnoli and J. J. Sheridan II, J. of Less-Common Met., 74:323 (1980).

21. Q. Z. Hong and F. M. d'Heurle, J. Appl. Phys., 72 9:4036 (1992).

22. E. P. Butler and P. R. Swann, In-Situ High Voltage Microscope Observations of Precipitation in Physical Aspects of Electron Microscopy and Microbeam Analysis edited by B. M. Siegal and D. R. Beaman (J. Wiley \& Sons, N. Y., London, Sidney, 1975) p. 131.

23. M. Y. Song, M. Pezeat, B. Darriet, P. Hagenmuller, J. of Materials Science, 20:2958 (1985).

24. B. Viegeholm, J. Kjøller, B. Larsen, and A. S. Pedersen, J. of Less-Common Met., 89:135 (1983).

25. A. S. Pedersen, K. Jensen, B. Larsen, and B. Vigeholm, J. of Less-Common Met., 131:31 (1987).

26. A. K. Altunoglu, D. A. Blackburn, N. St. J. Braithwaite, and D. M. Grant, J. of LessCommon Met., 172-174: (1991).

27. J. J. Reilly and R. H. Wiswall, Inorg. Chem., 7:2254 (1968).

28. Ultramet Corporation, Pacoima, California 91331.

29. Goodfellow Corporation, Berwyn, PA 19312.

30. Prof. E. J. Lavernia, Chemical Engineering Department, University of California, Irvine, Irvine, CA 92717.

31. Magnesium Elektron, Manchester, England.

32. A. Sieverts : Z. Physik. Chem. 88:451 (1914). 
33. S. Dushman, Scientific Basis of Vacuum Technique (J. Wiley and Sons, New York, London, Sydney, 1962) p. 692.

34. M. Y. Song, E. Ivanov, B. Darriet, M. Pezat, and P. Hagenmuller, J. of Less-Common Met., 131:71 (1987).

35. A. A. Nayeb-Hashemi and J. B. Clark, eds., Phase Diagrams of Binary Magnesium Alloys (ASM Intl., Ohio, 1988).

36. S. Housh, B. Mikucki, and A. Stevenson, Properties of Magnesium Alloys (ASM MetalsHandbook, 10th Ed. v2., 1990) p. 480. 


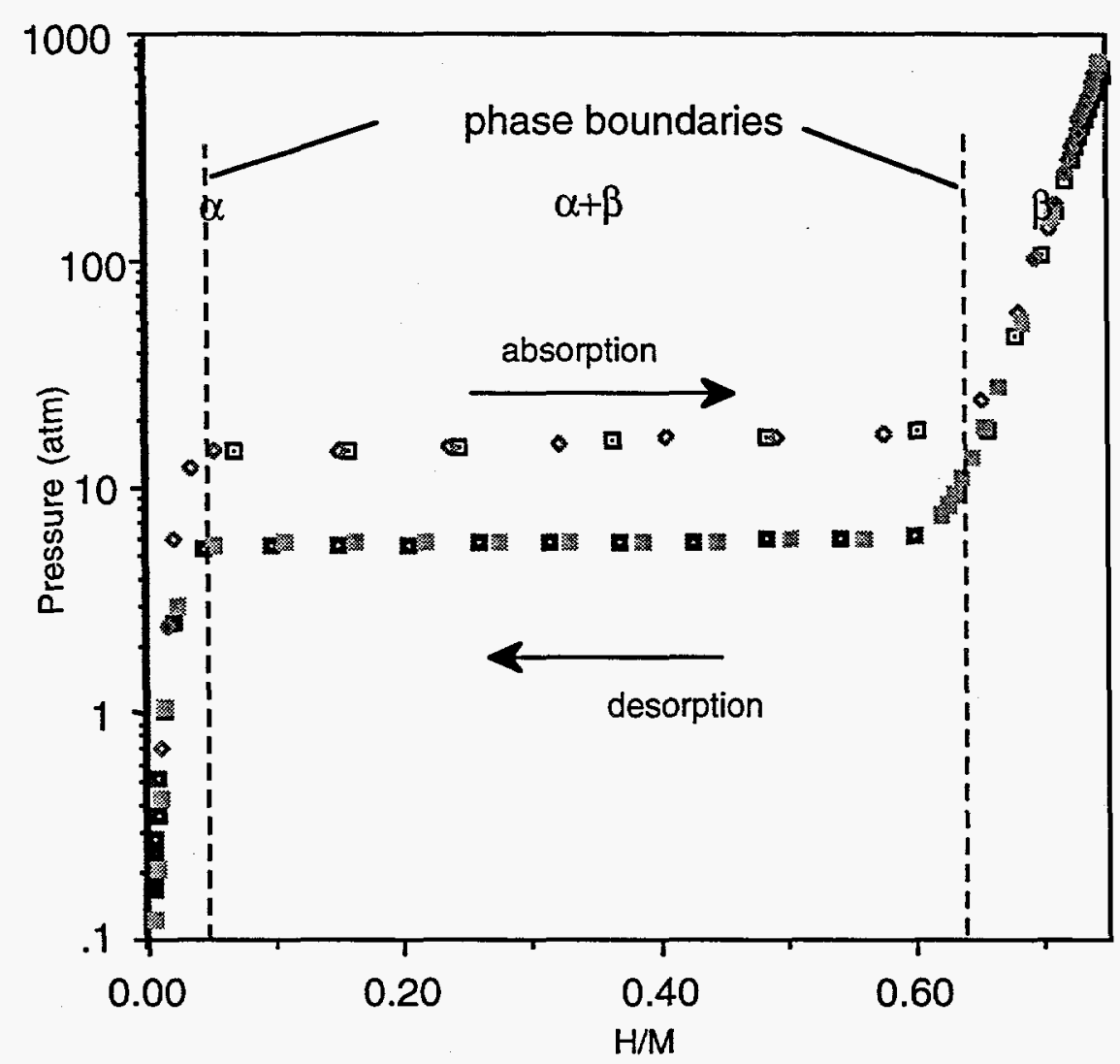

Figure 1. A PCT plot of an ideally behaving hydride is characterized by a large plateau region over which the pressure does not change as the hydride phase concentration increases. The extremes of the plateau mark the concentration limits of the hydride phase which are roughly the same for the absorption and desorption curves. These two curves result at each temperature due to a hysteresis effect. 


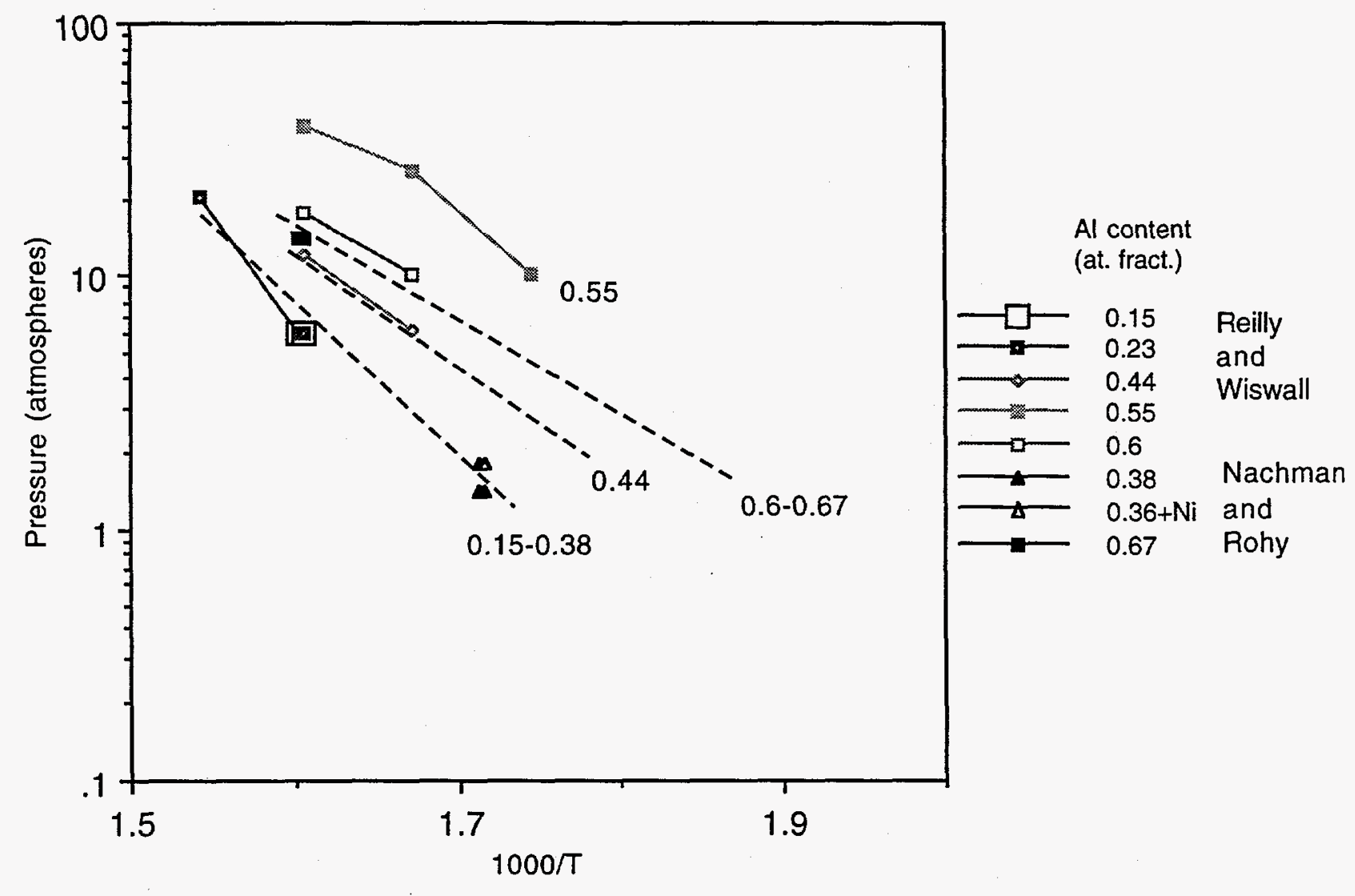

Figure 2. A van't Hoff plot of previously investigated $\mathrm{Mg}$-Al alloys reflect the effect of $\mathrm{Al}$ on the basic $\mathrm{Mg}$ hydride behavior. The alloy at 0.55 atom fraction is particularly attractive due to its high pressure behavior. 


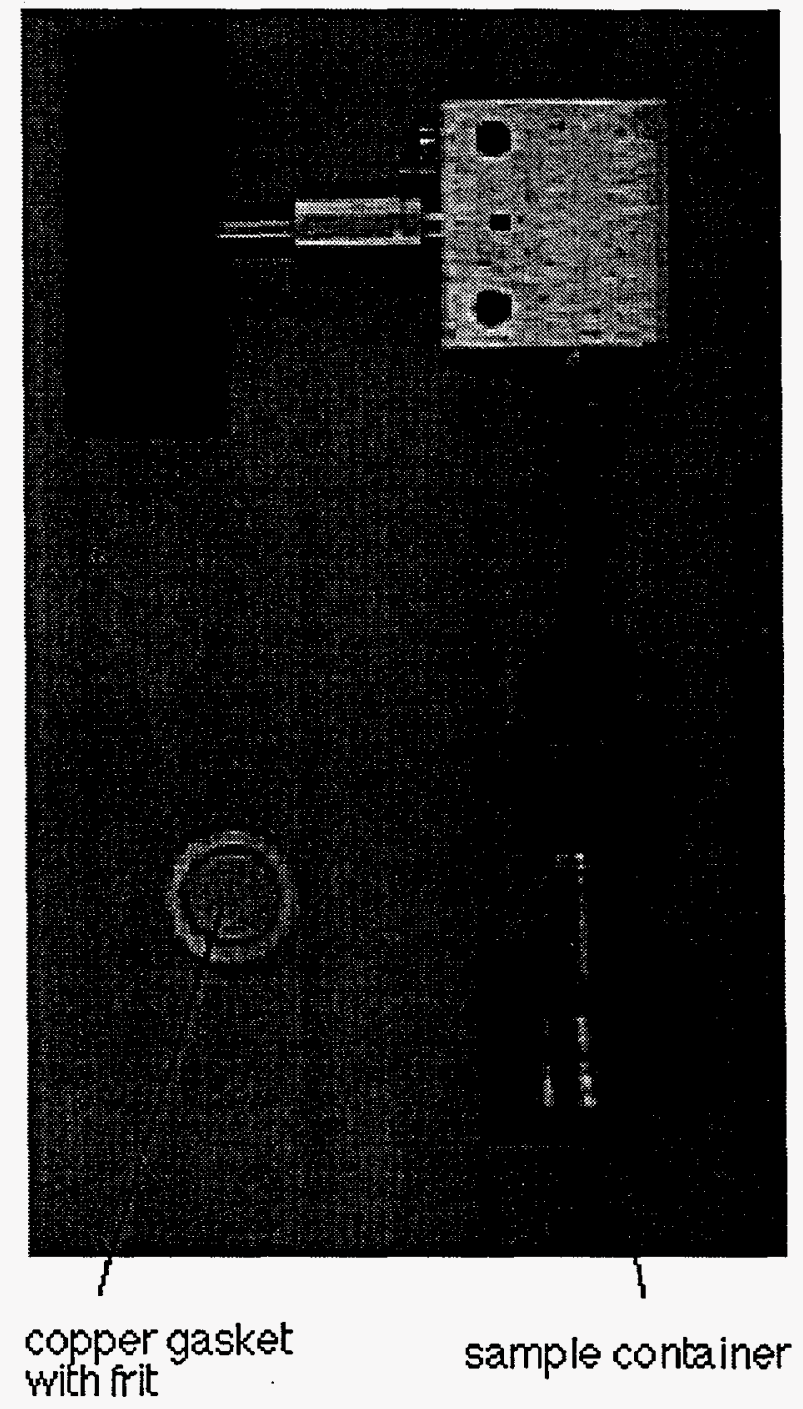

Figure 3. A photograph of one of the sample holders. The material is contained within a cylindrical recess within the container. The frit is placed over the recess to prevent particulate transport. 


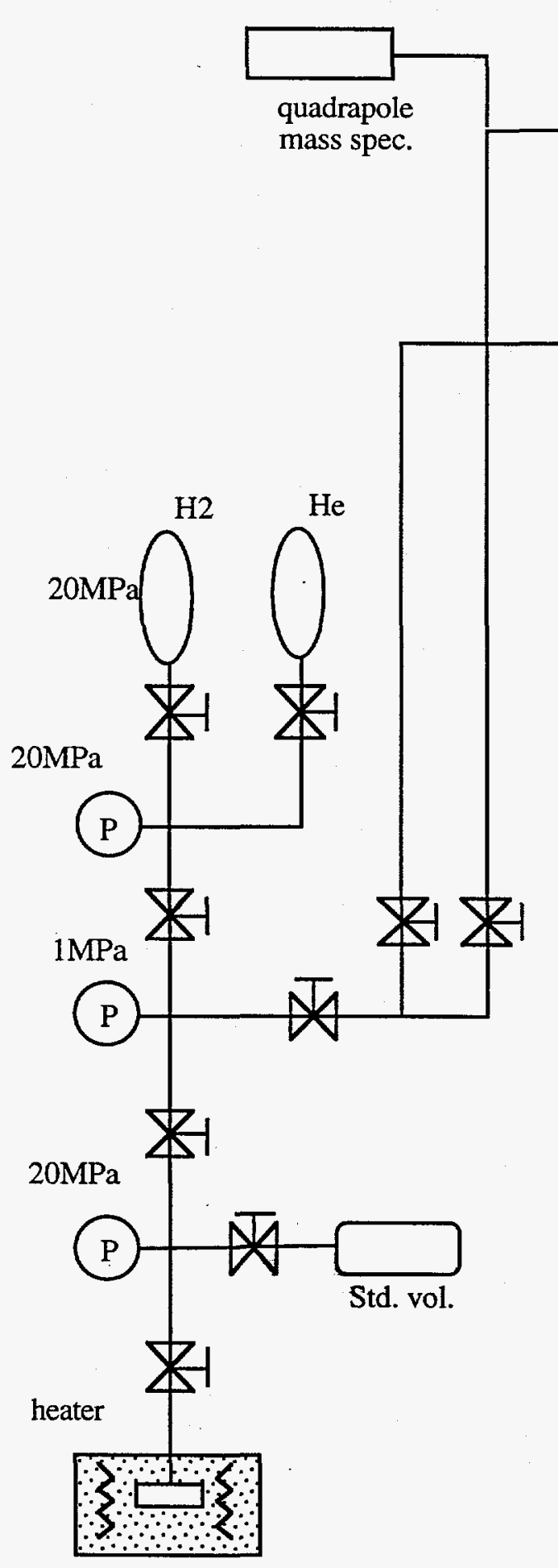

High Pressure System high vacuum

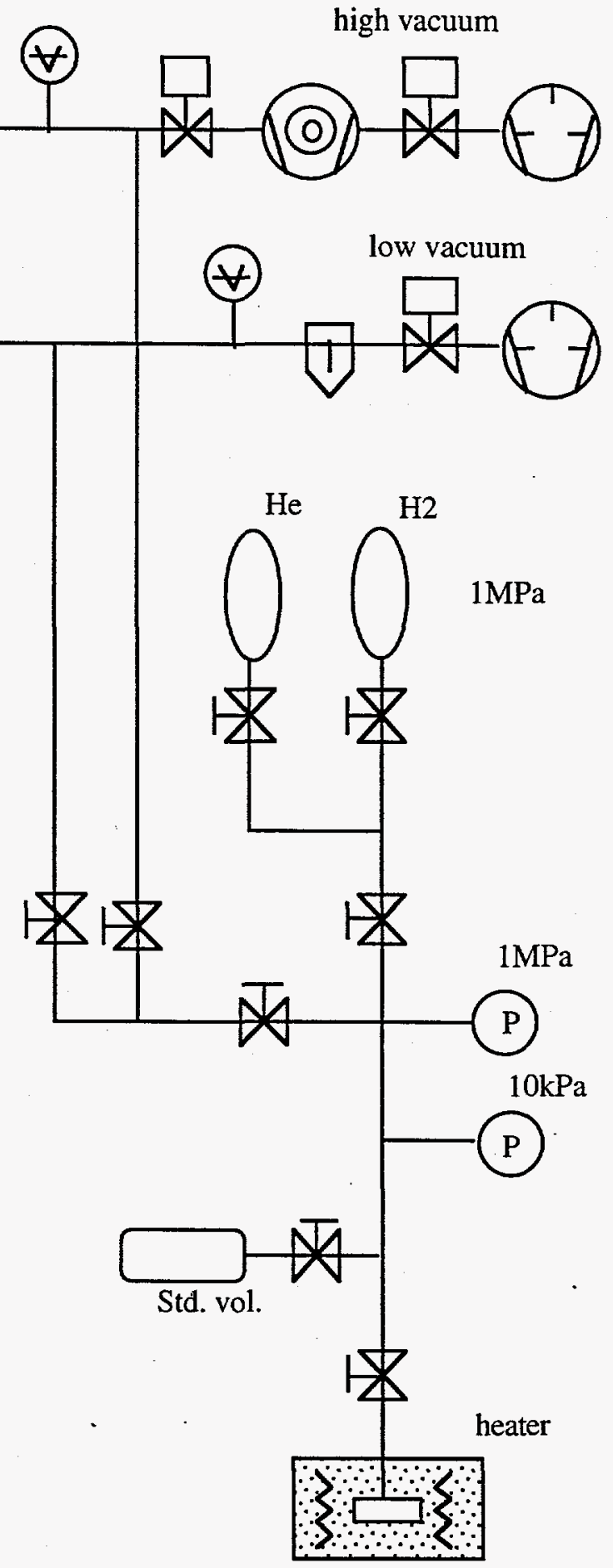

Low Pressure System

Figure 4. Schematic diagram of the Sieverts' gas system manifold. It was constructed using all metal components. The two stations provided pressure measurement capability from 10-1 to $20 \mathrm{Mpa}$. Two vacuum systems provide evacuation at nominal base pressures of $10^{2}$ and $10^{-7} \mathrm{~Pa}$. 


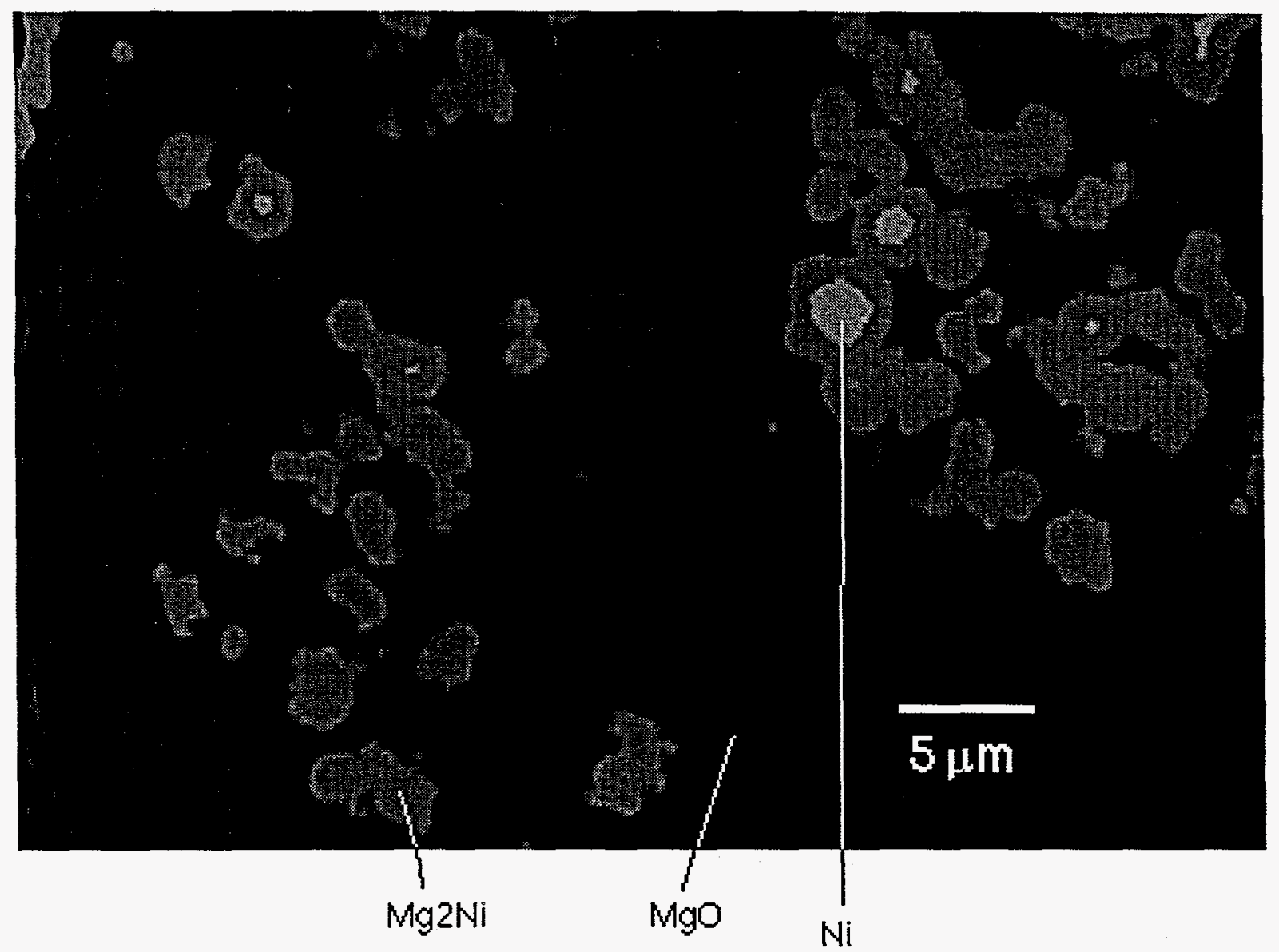

Figure 5. Electron micrograph showing the in-situ formation of $\mathrm{Mg}_{2} \mathrm{Ni}$. Particle sizes are roughly dictated by the size of the initial $\mathrm{Ni}$ particles. The new phase forms around the $\mathrm{Ni}$ core and $\mathrm{MgO}$ shells are only the remnants of the initial $\mathrm{Mg}$ particles. 


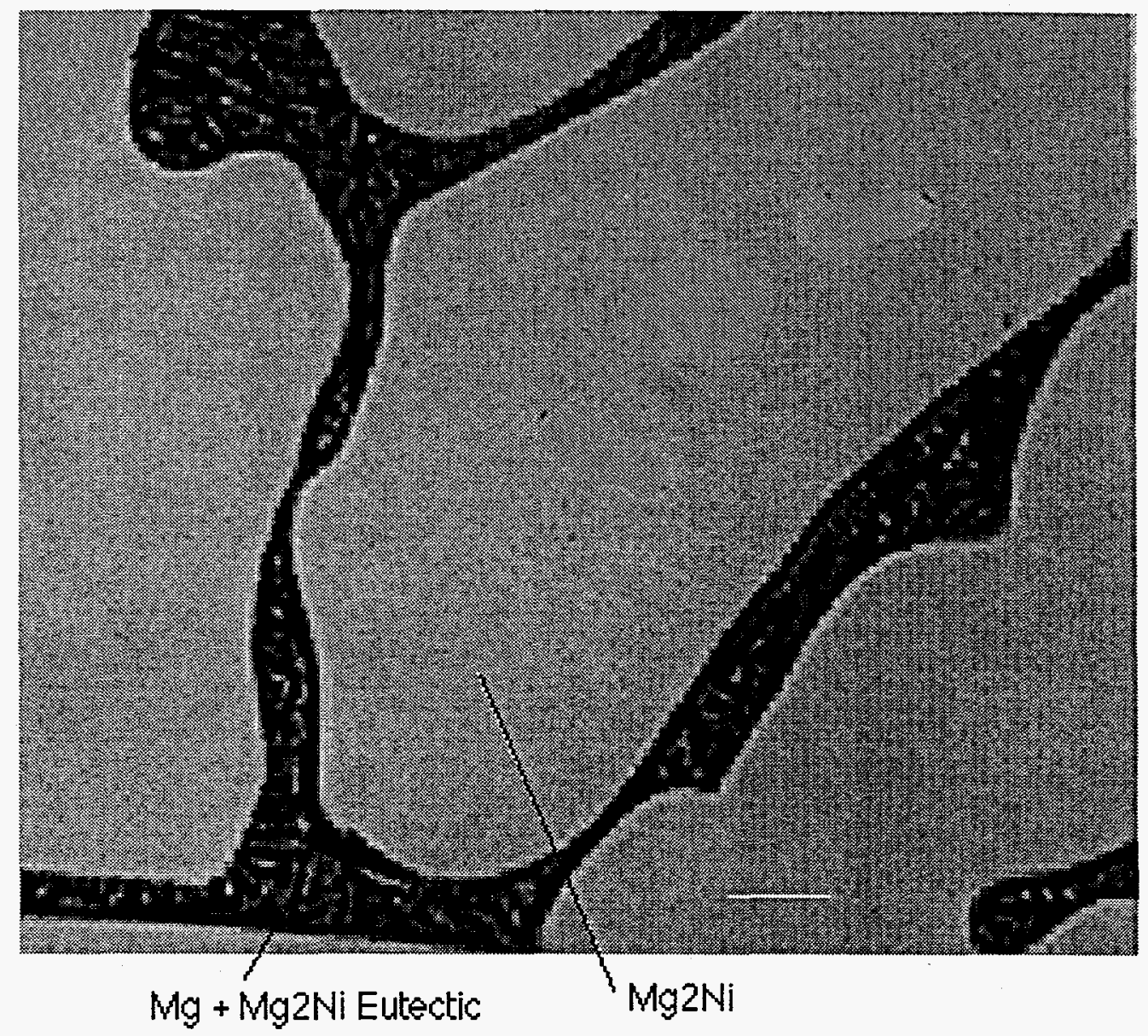

Figure 6. $\mathrm{Mg}_{2} \mathrm{Ni}$ formed by ingot metallurgy typically has a eutectic and phase present at the interfaces of the predominately $\mathrm{Mg}_{2} \mathrm{Ni}$ matrix. 


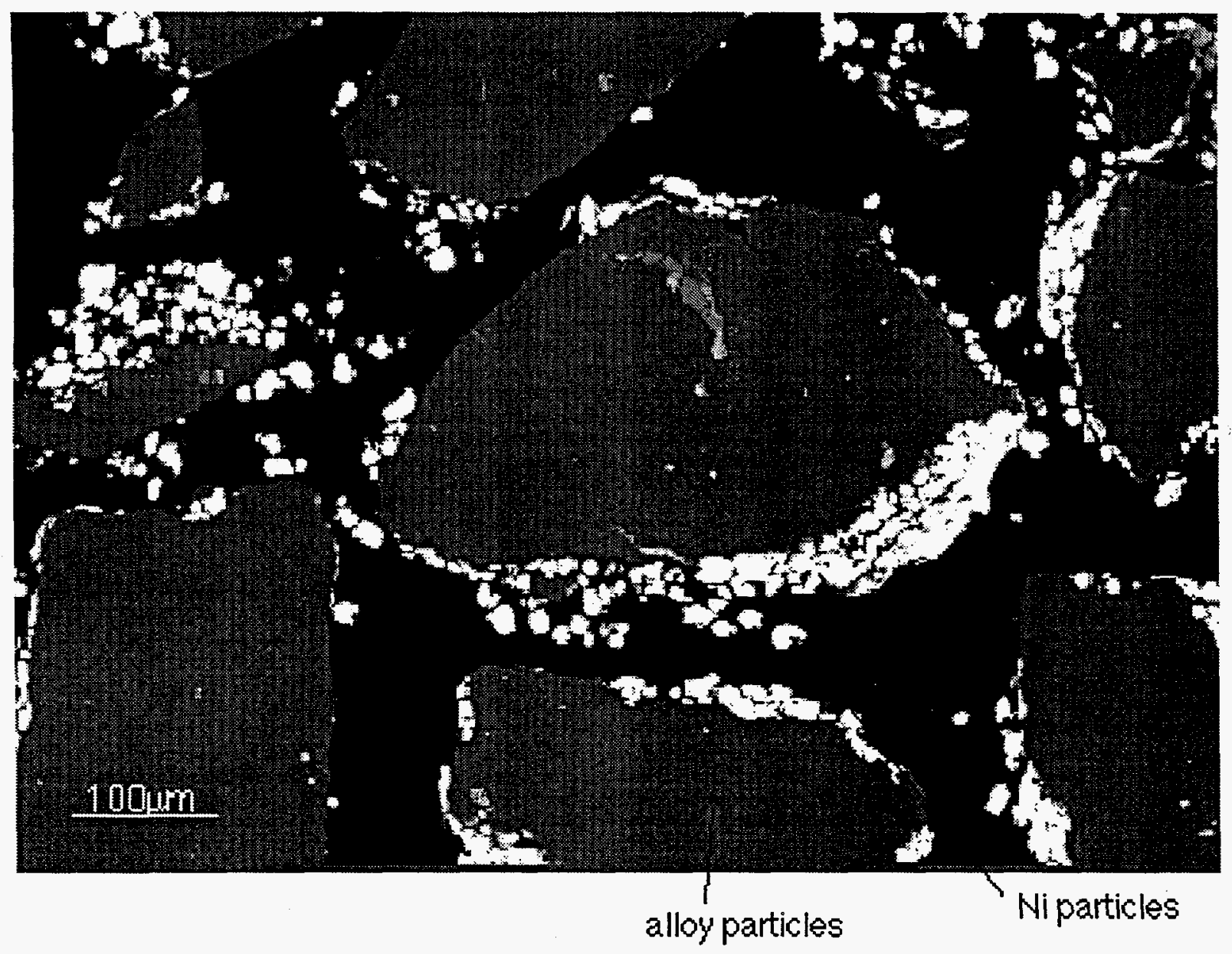

Figure 7. Simple mixing resulted in relatively sparse distribution of $\mathrm{Ni}$ on alloy particles. In the cases with the most contact, particles agglomerated to the surface as seen in the micrograph. 


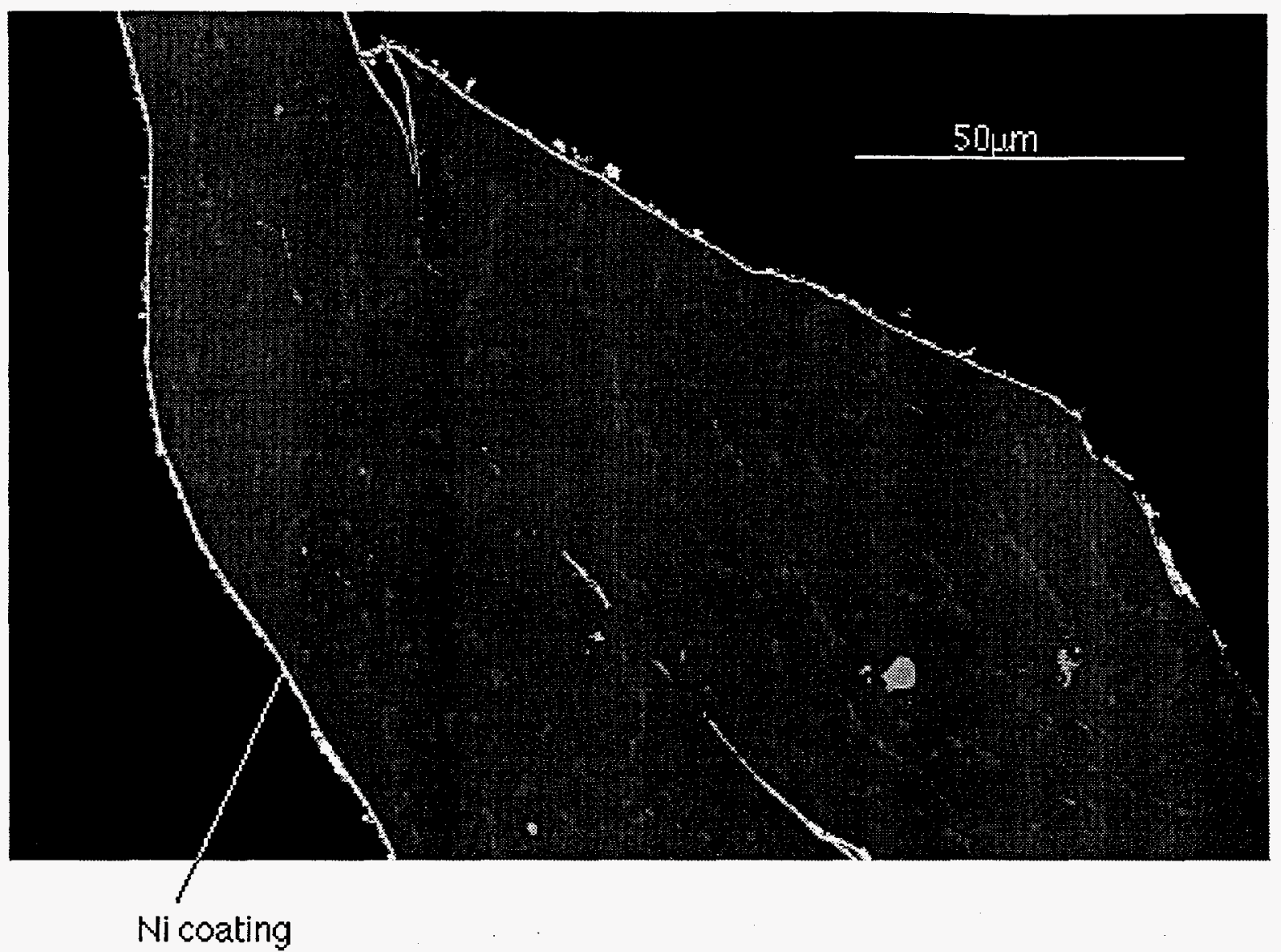

Figure 8. A very uniform coating of Ni on alloy particles is provided by a commercial gas-phase process. Note the penetration of the coating into the crack in the upper left of the figure. 


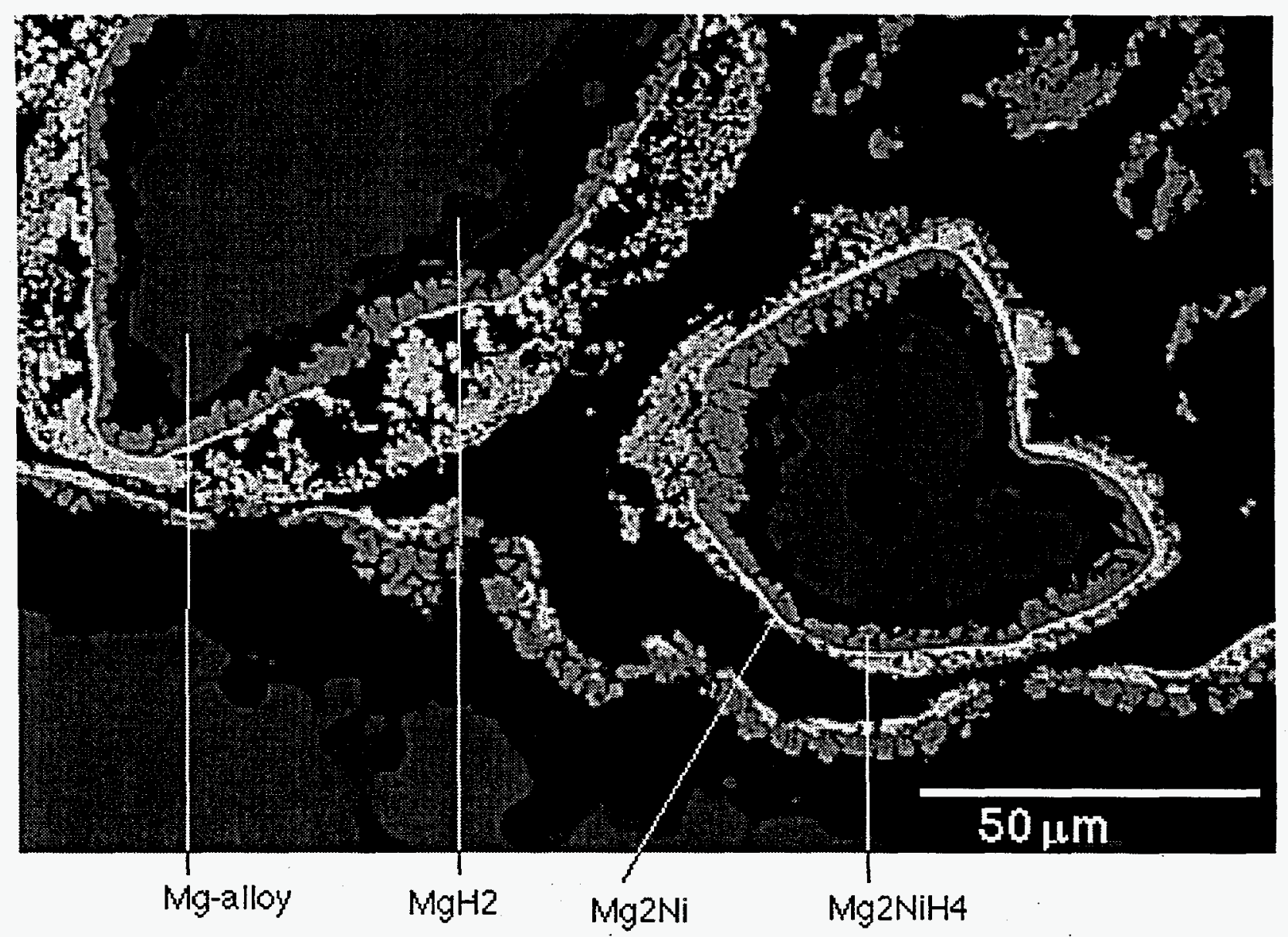

Figure 9. The reaction between $\mathrm{Mg}$ in the alloy particles and the $\mathrm{Ni}$ coating to form $\mathrm{Mg}_{2} \mathrm{Ni}$ caused a delamination of the coating during hydriding. The resulting separation did not permit effective transport of $\mathrm{H} 2$ to the bulk and the kinetics were not improved. 


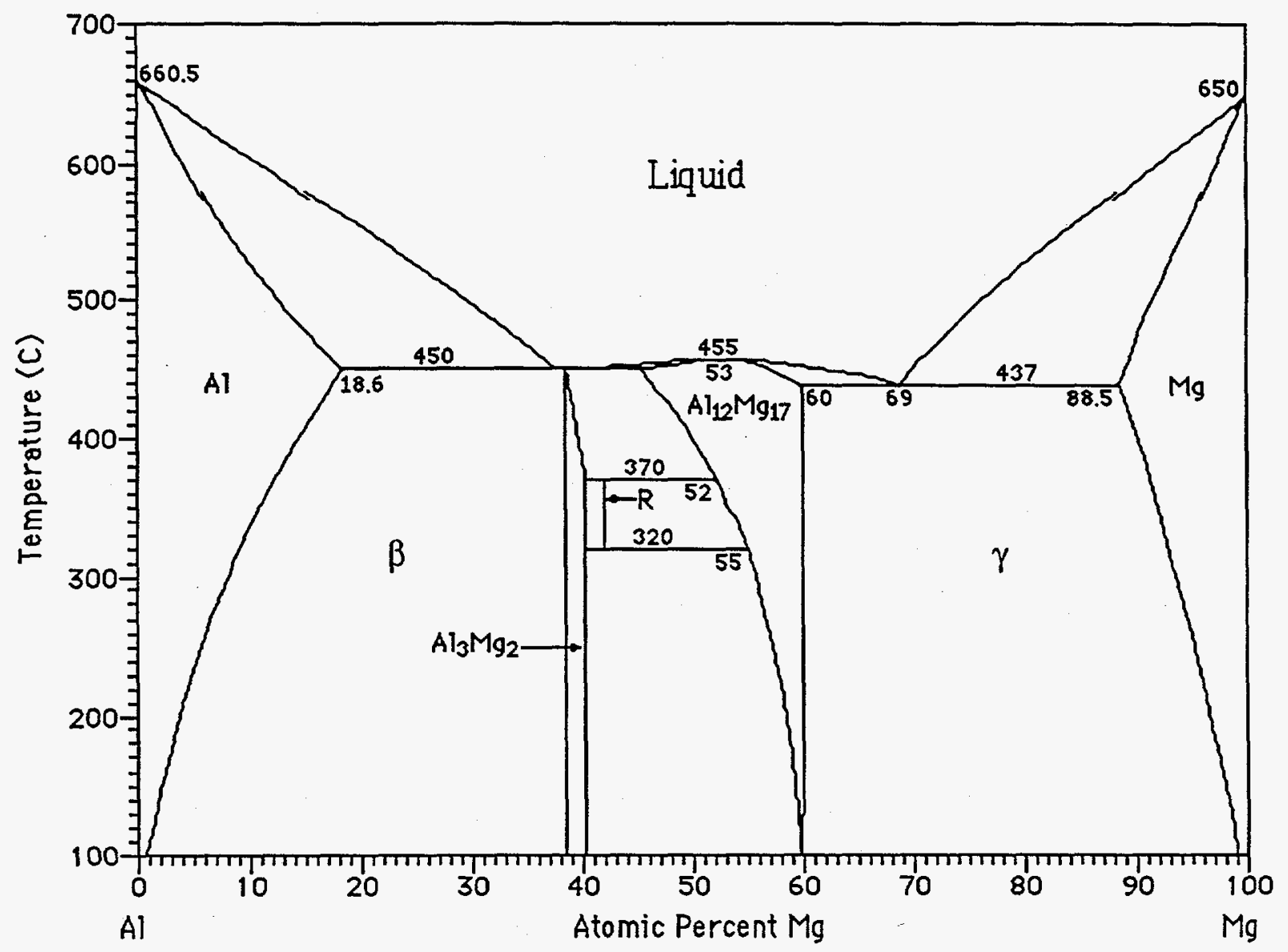

Figure 10. The Mg-Al phase diagram was used as an initial guide for choosing alloy materials. 


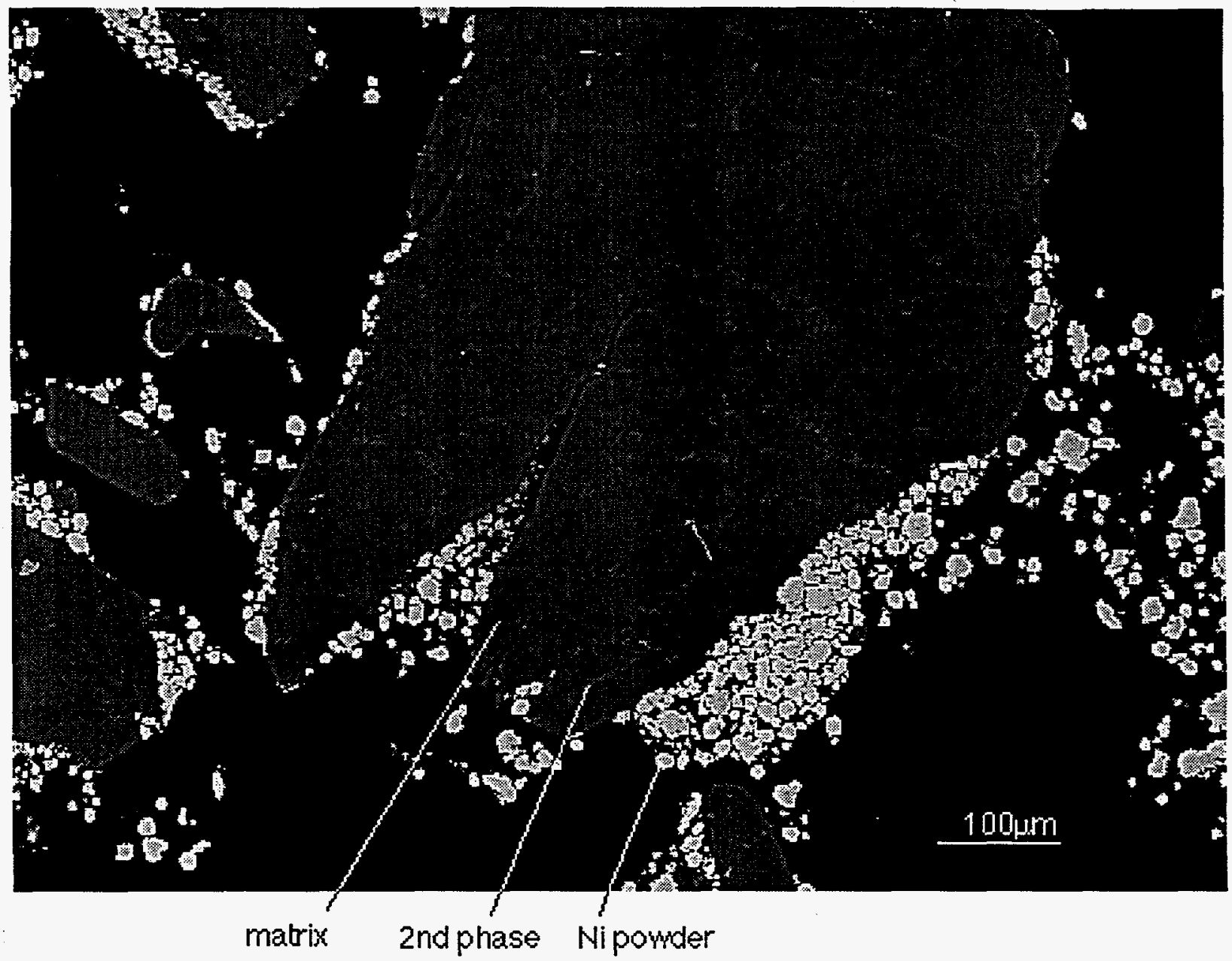

Figure 11. Turnings of the AZ31 material were mechanical mixed with Ni powder and resulted in some agglomeration of the Ni particles on the surface of the particles. A larger particle is shown to reveal the additional 2nd phase structure more clearly. 


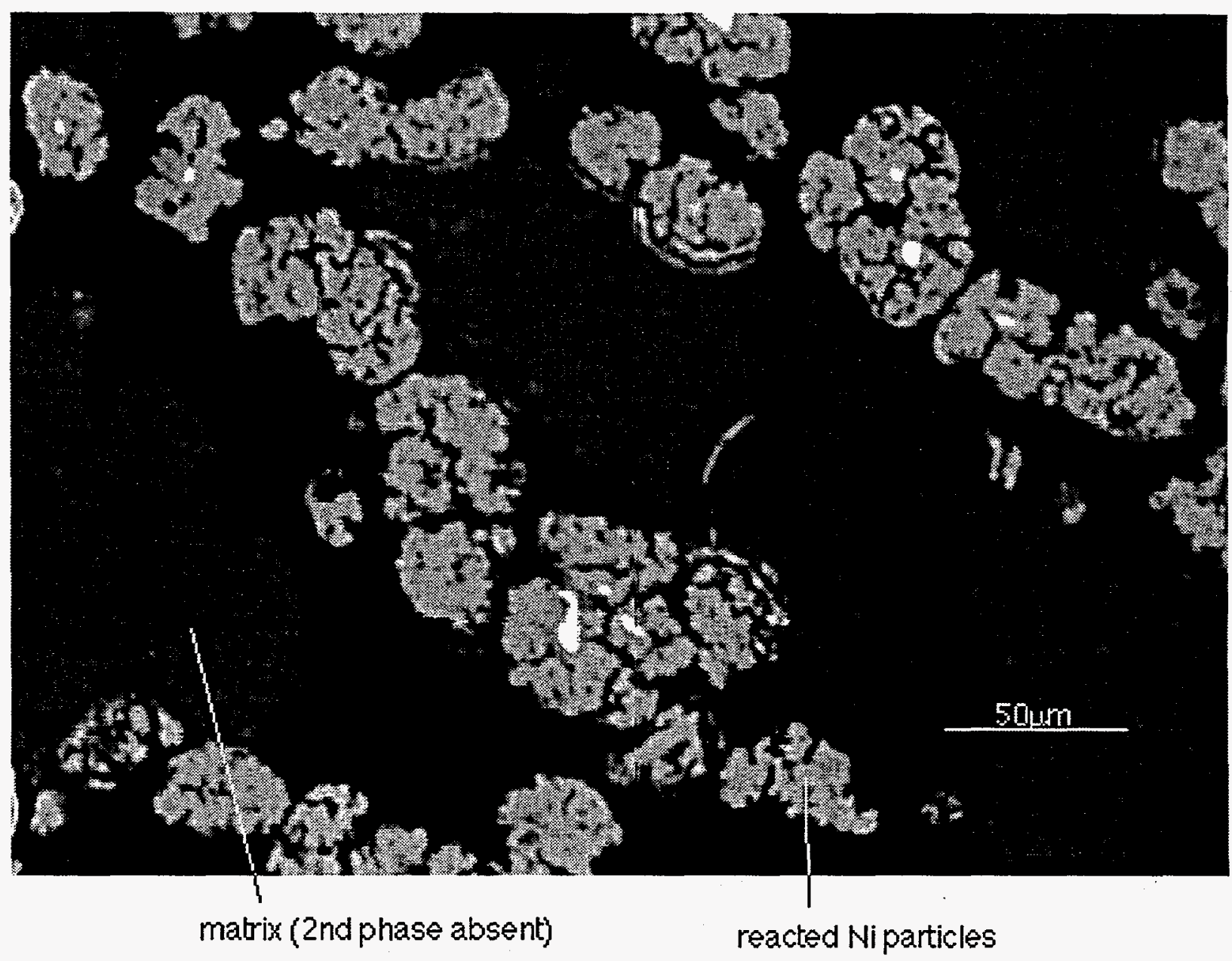

Figure 12. AZ 31 particles were altered after 200 hours at $300^{\circ} \mathrm{C}$ in hydrogen environment. $\mathrm{Mg}_{2} \mathrm{Ni}$ reaction on $\mathrm{Ni}$ particles is nearly complete and $2 \mathrm{nd}$ phase is absent. 


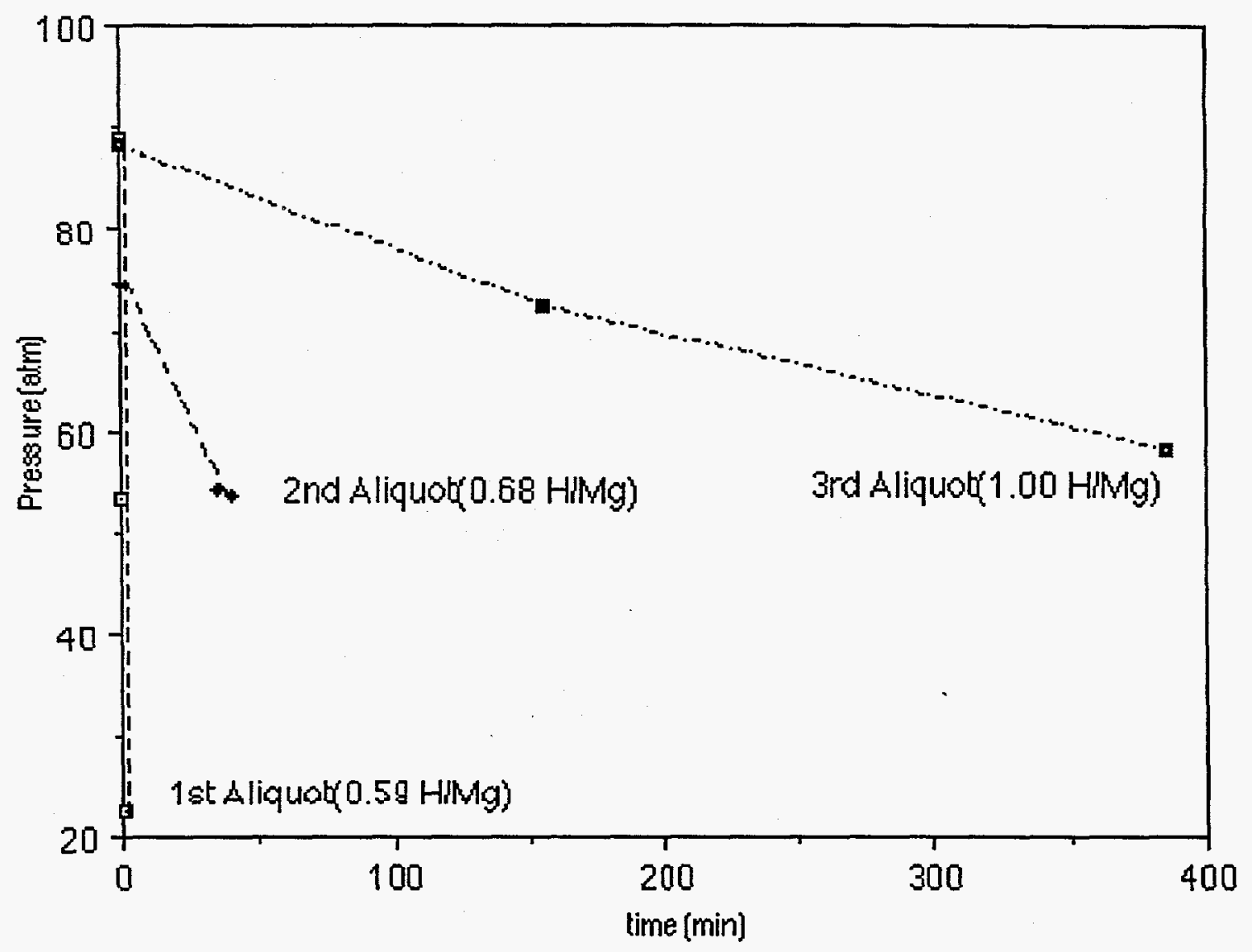

Figure 13. The $\mathrm{H}_{2}$ pressure vs. time is shown for a loading of the AZ31 sample (4th cycle, $300^{\circ} \mathrm{C}$ ). The time increases significantly as the loading approaches an $\mathrm{H} / \mathrm{Mg}$ of 1.0 . 


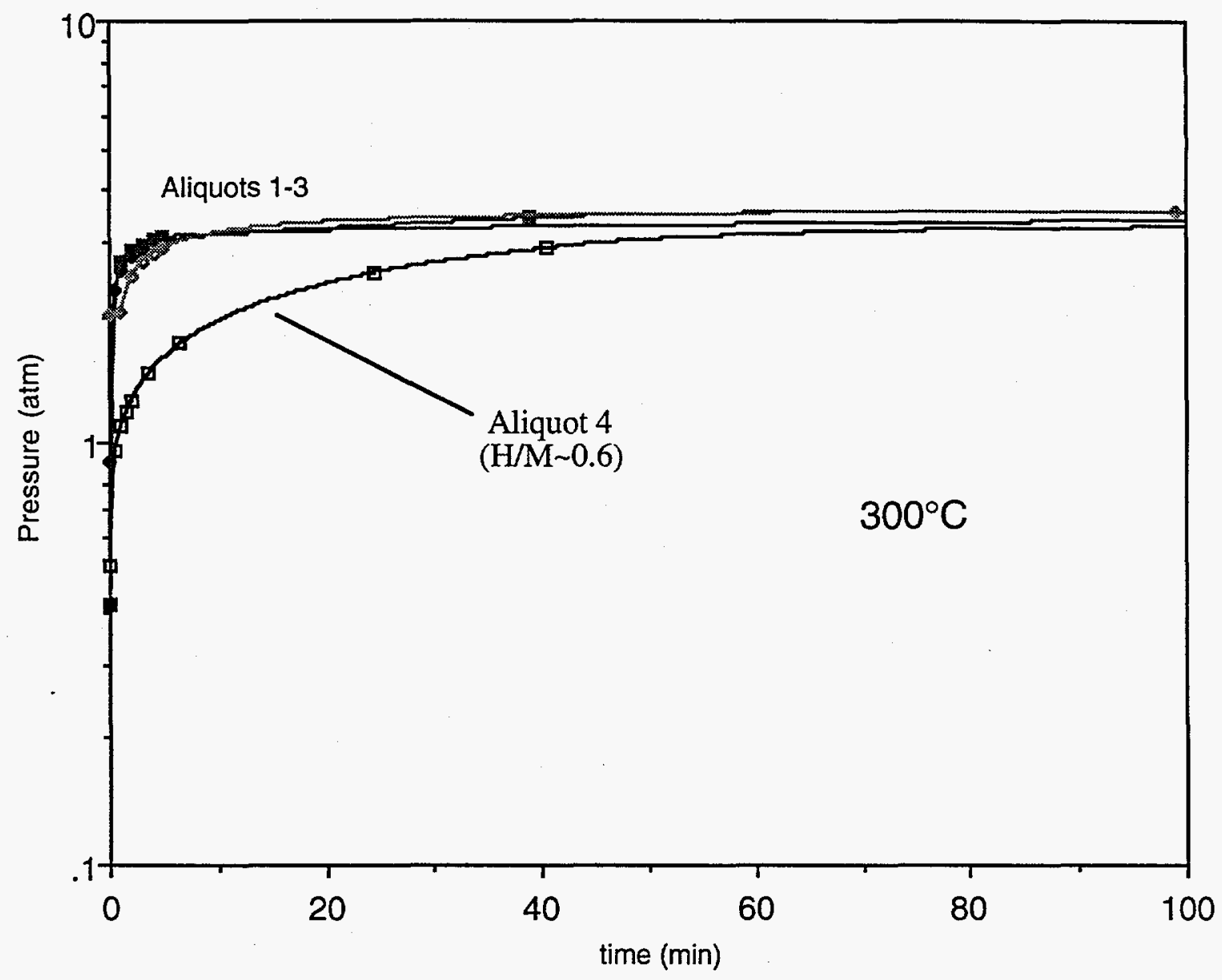

Figure 14. The $\mathrm{H}_{2}$ desorption pressure with time for $\mathrm{AZ} 31$ (4th cycle, $300^{\circ} \mathrm{C}$ ). Aliquot 4 is taken near an $\mathrm{H} / \mathrm{Mg}$ ratio of 0.6 and indicates the extent of the $\mathrm{Mg}-\mathrm{Ni}$ phase. 


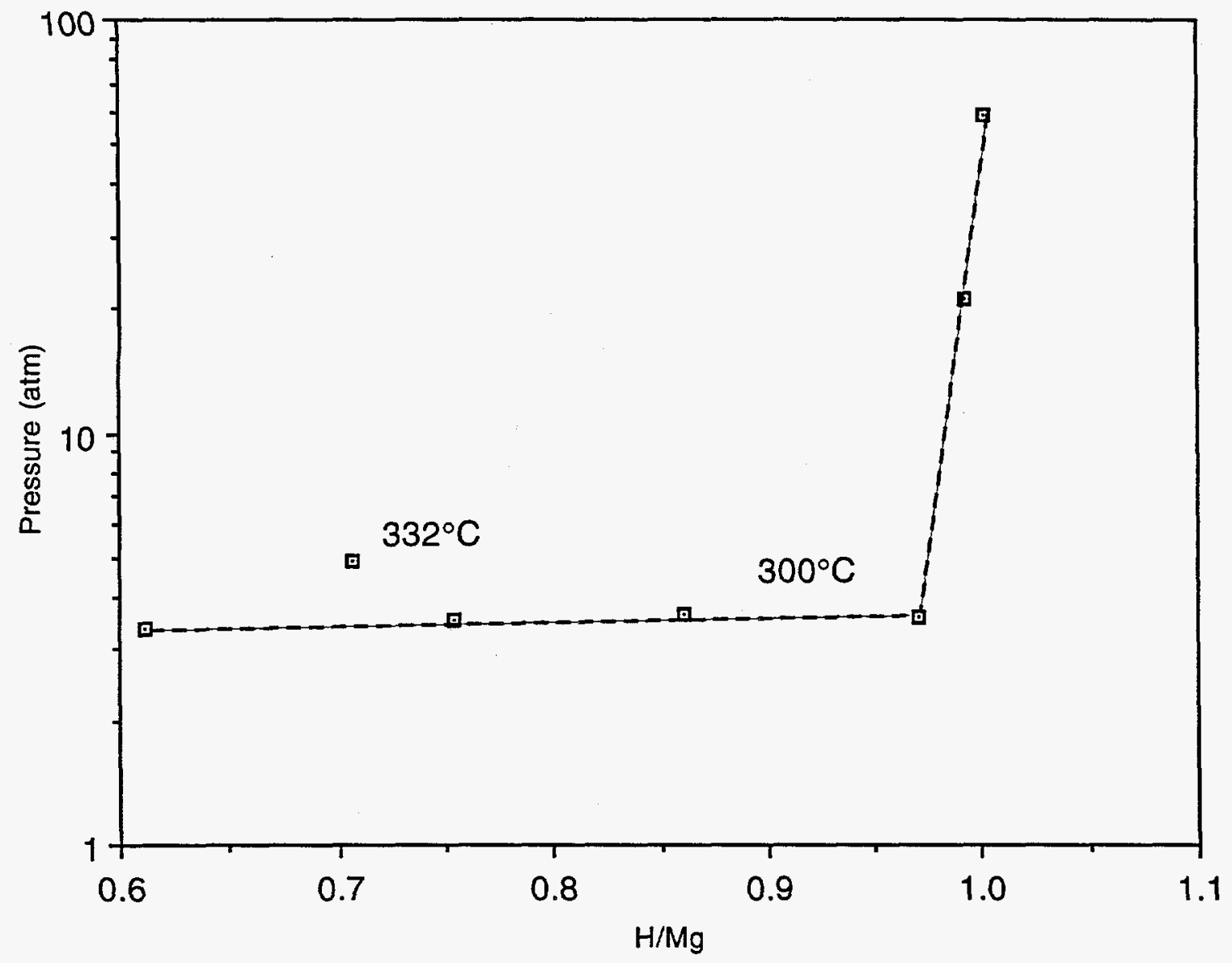

Figure 15. PCT isotherm and plateau region of $\mathrm{AZ31}$. Sample was heated to $332^{\circ} \mathrm{C}$ and returned to $300^{\circ} \mathrm{C}$ during this run to insure that the plateau was reproducible. 


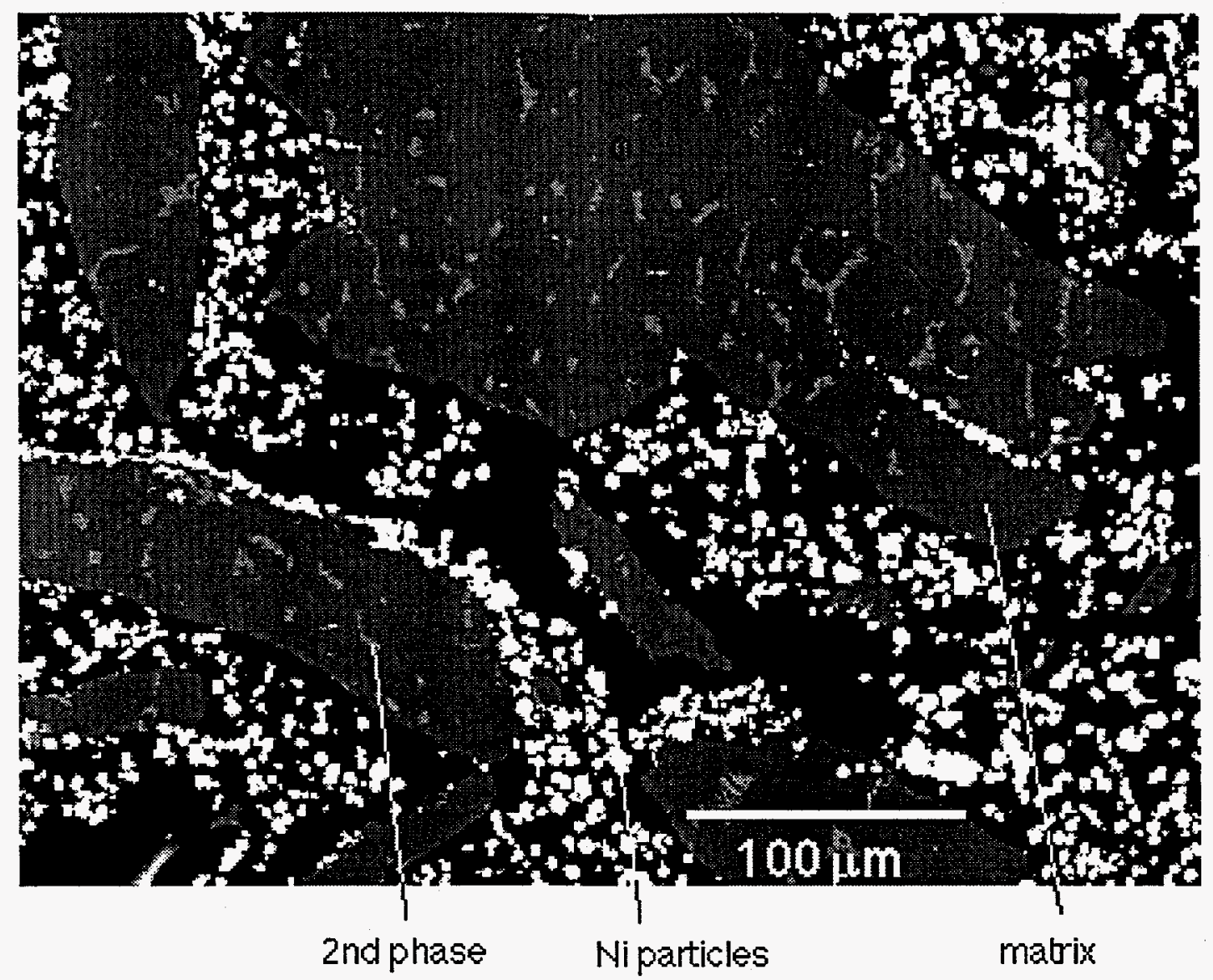

Figure 16. AZ 61 alloy mixed with Ni particles were very similar to AZ 31 sample. Again some of the Ni particles were agglomerated on the surface of the alloy. 

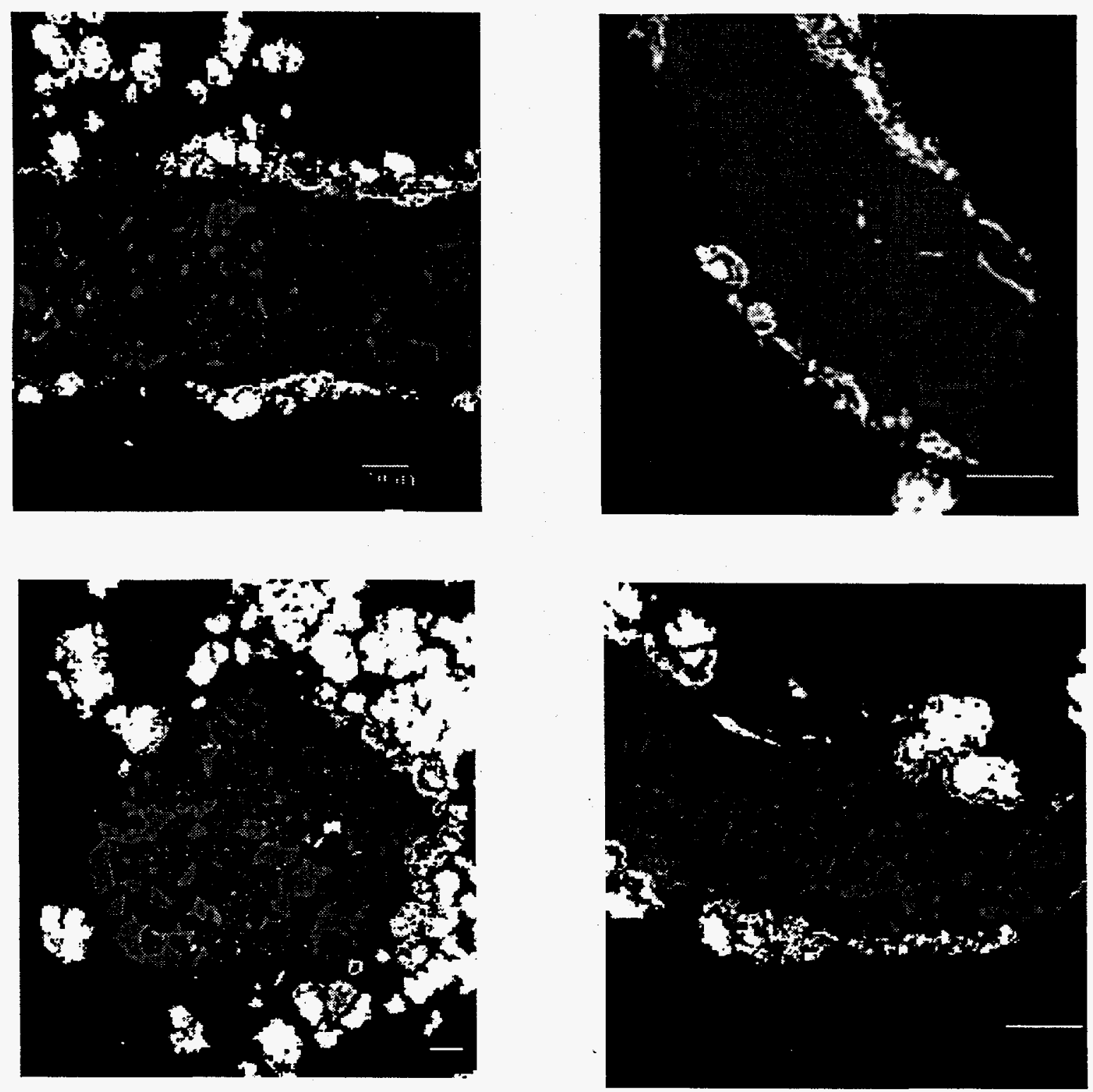

Figure 17. Typical microstructure of the transformed AZ 61 alloy which reflected a greater overall complexity than other samples that were examined. 


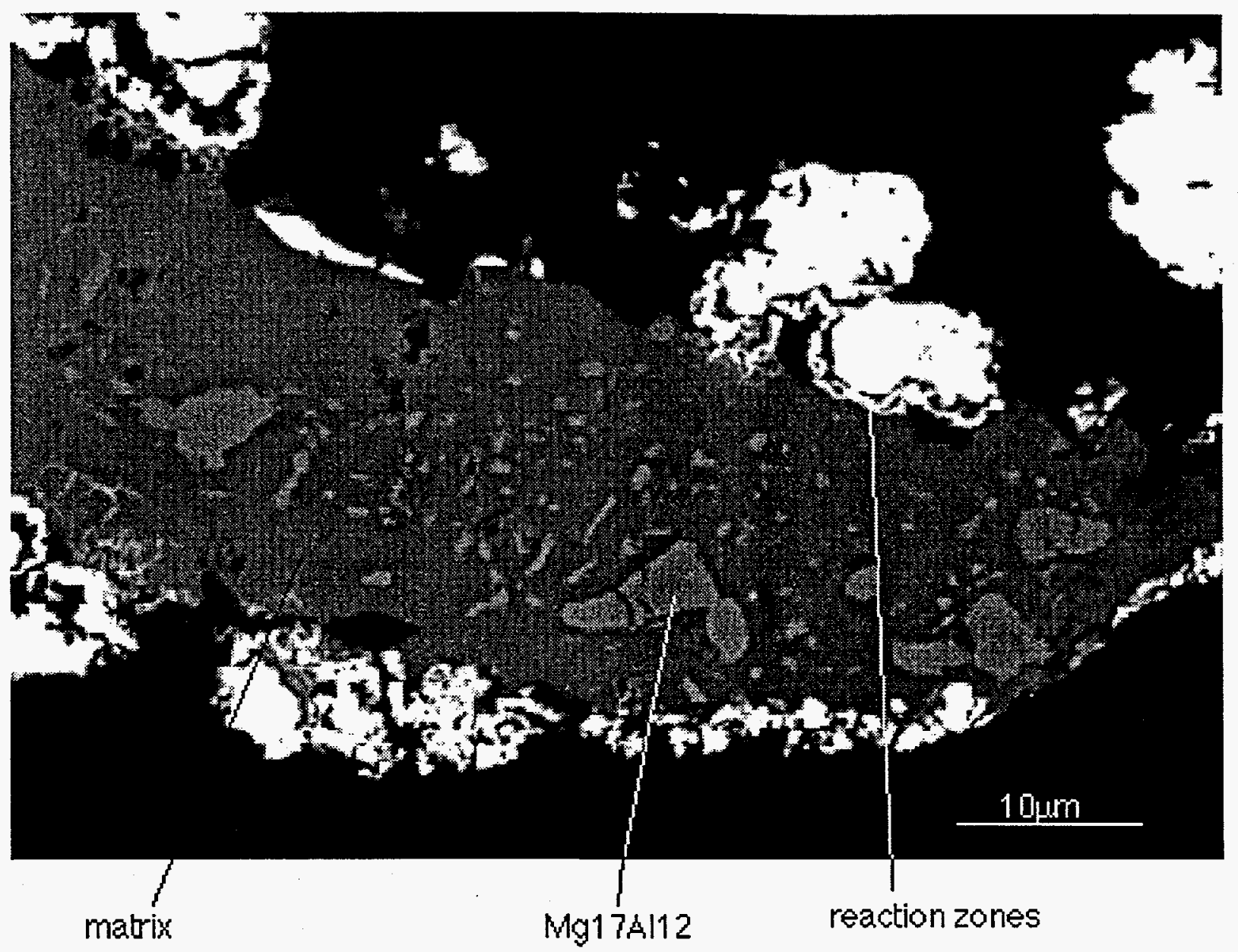

Figure 18. Microstructure of the transformed AZ61 alloy showed characteristic regions that were not present in other alloys. The lighter shaded regions are rich in $\mathrm{Ni}$ and indicate a reaction between the Ni particles in contact with the alloys surface. 


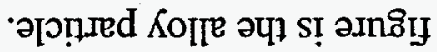

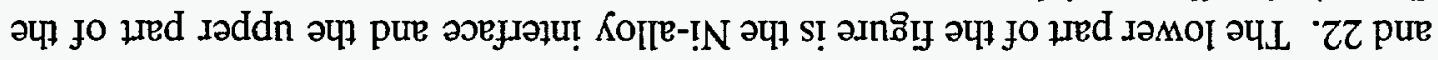

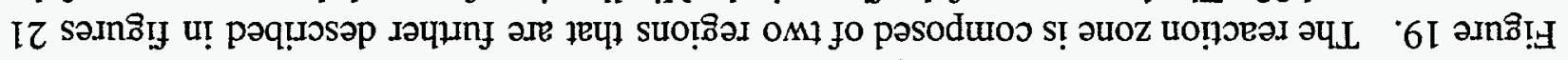

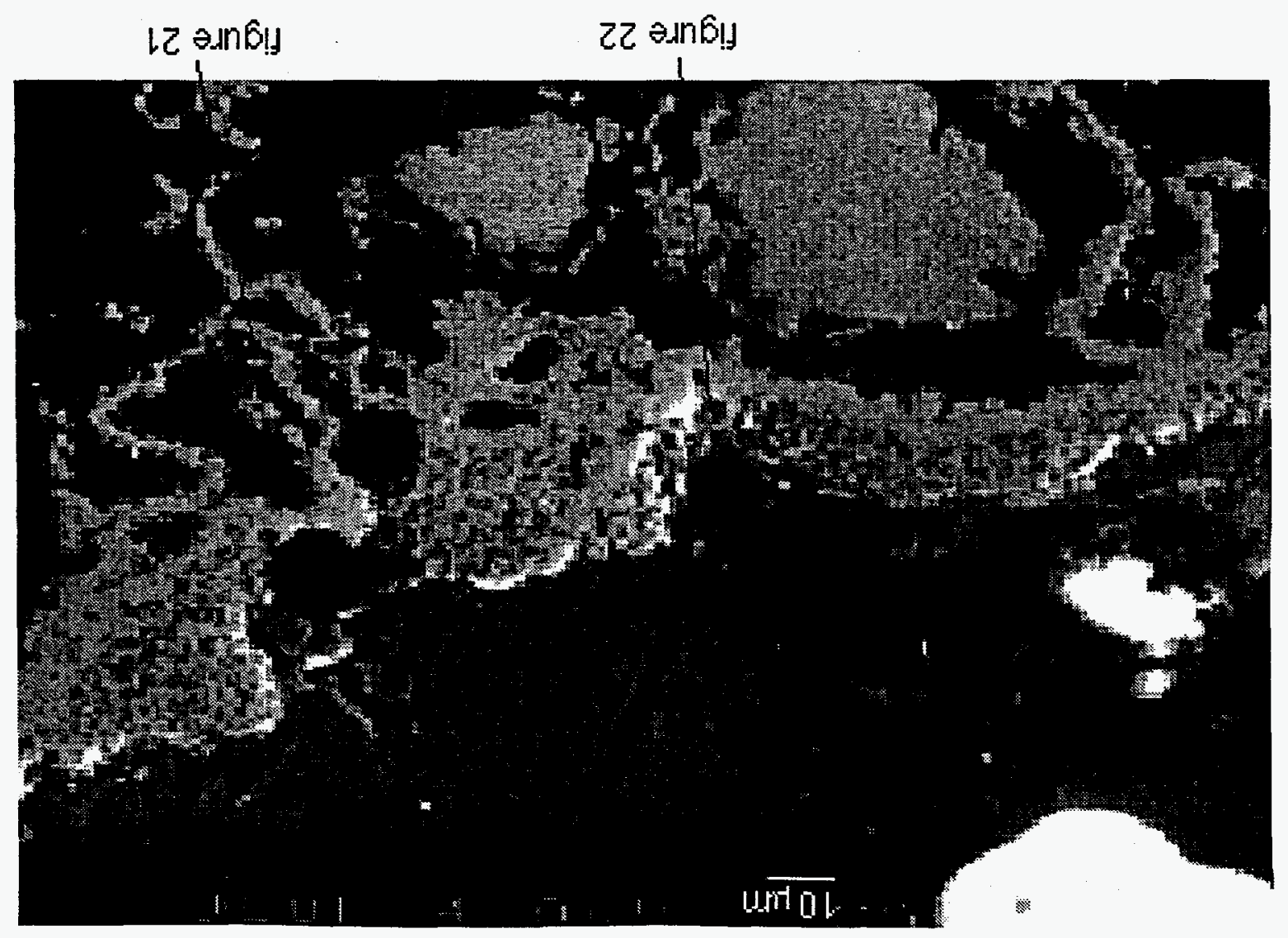




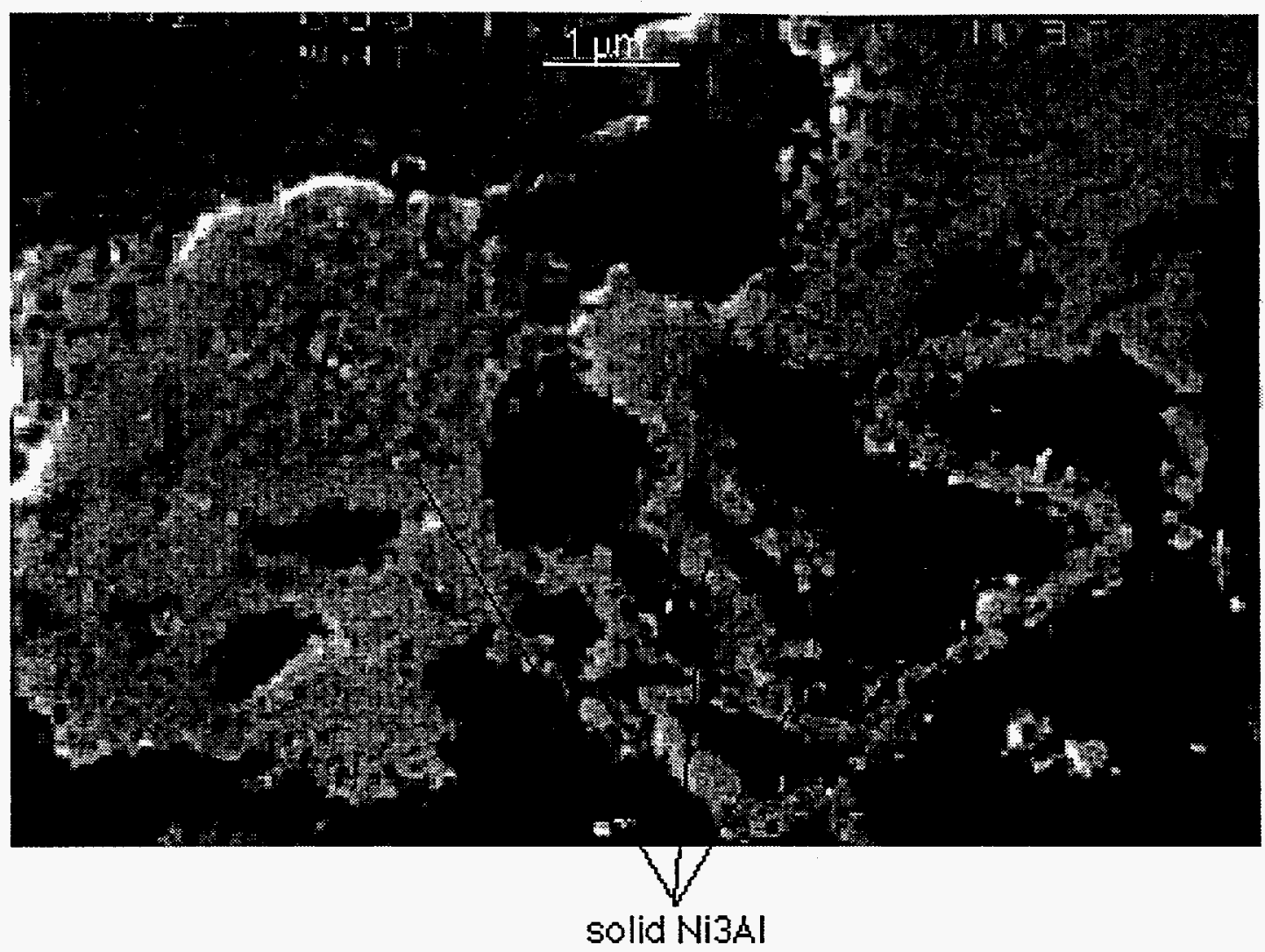

Figure 20. The outermost region of the interface is composed of ridges of $\mathrm{Ni}_{3} \mathrm{Al}$ where the $\mathrm{Ni}$ particles abutted the alloy. 


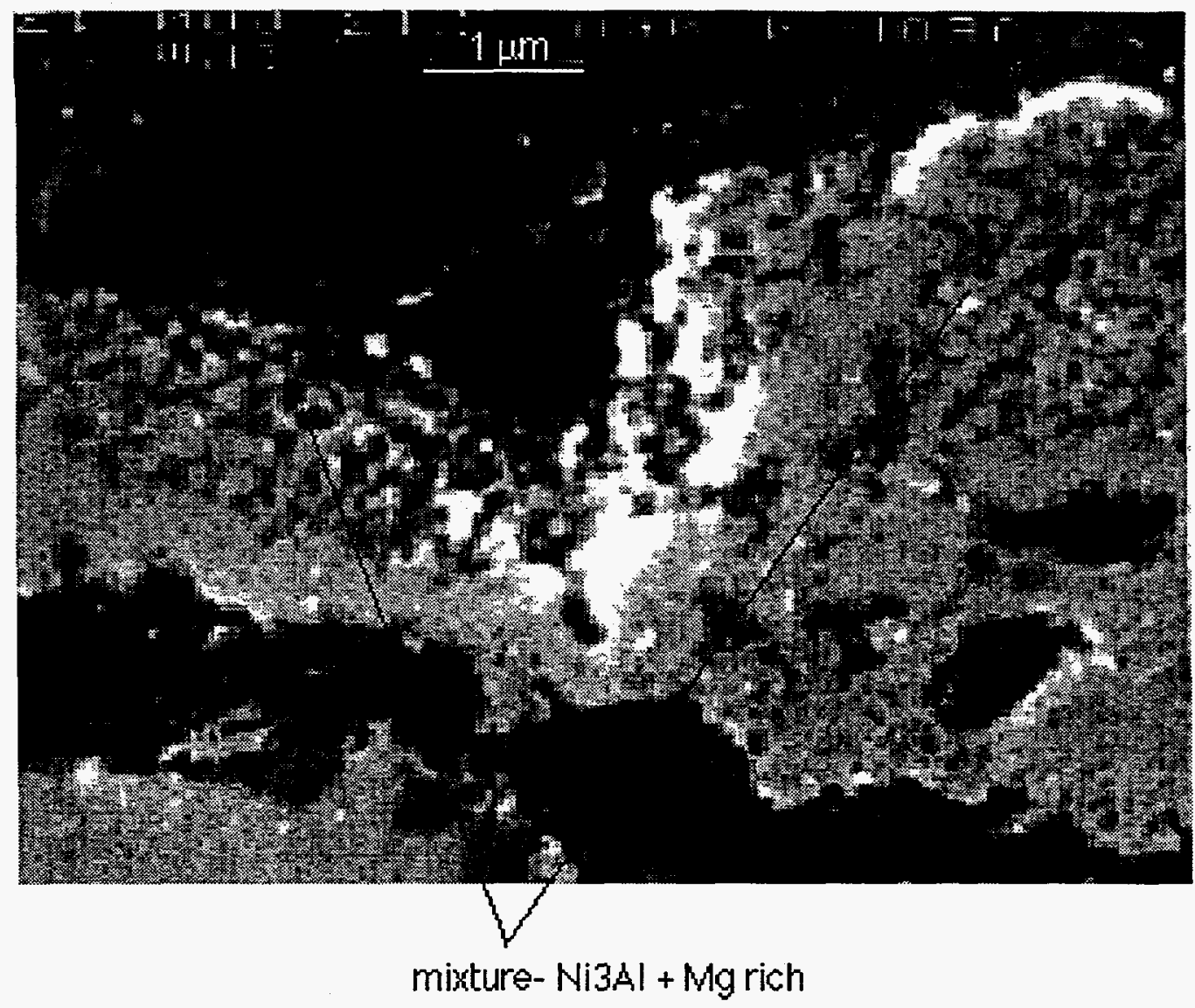

Figure 21. Near the bulk of the alloy particle, a mixture the $\mathrm{Ni}_{3} \mathrm{Al}$ and $\mathrm{Mg}$-rich phases were observed. In the micrograph, these features appear grainy in texture. 


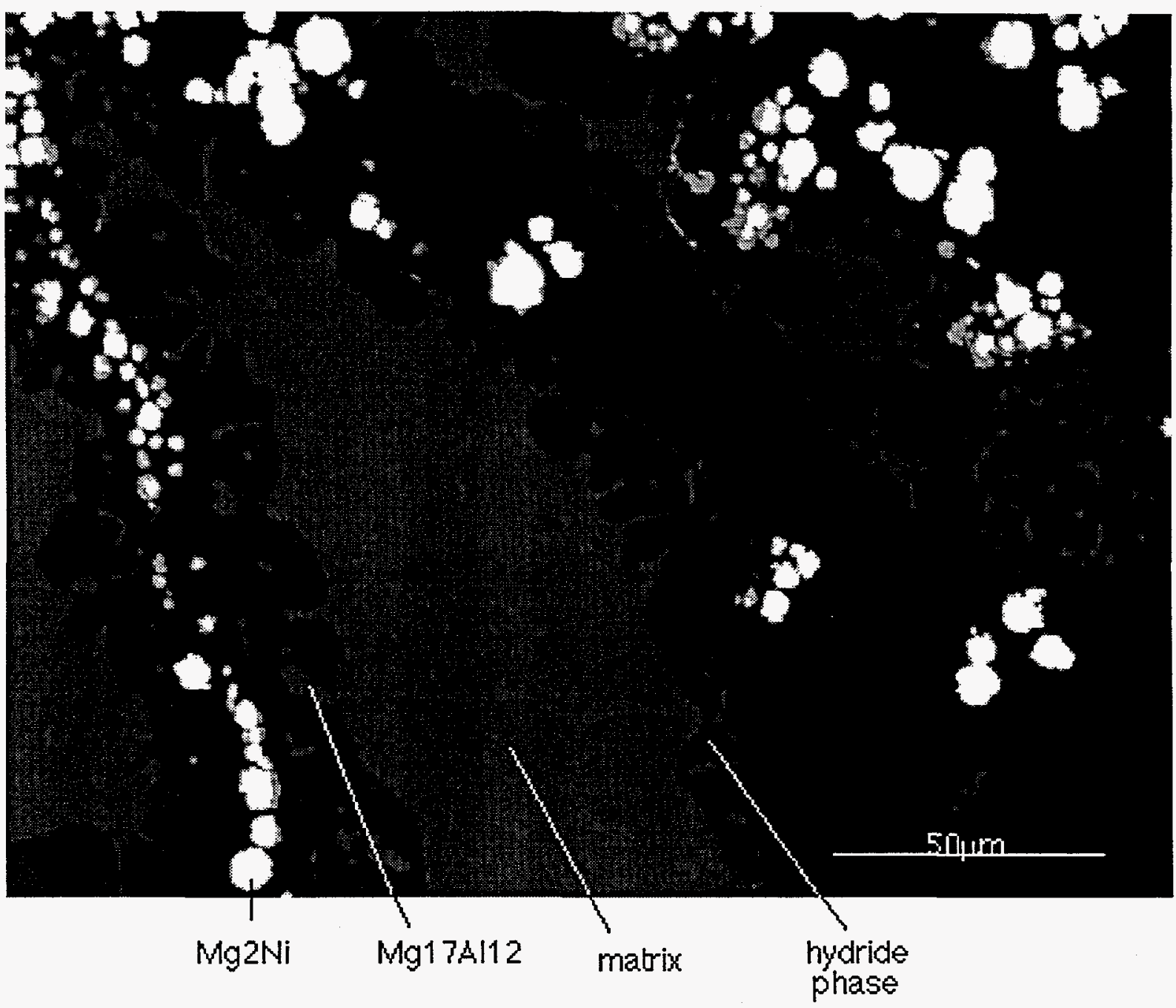

Figure 22. Micrographs of AZ61 samples that did not exhibit high pressure and fast kinetics did not show reaction zones. Hydride phases in the near-surface region which were retained after the measurements are visible. 


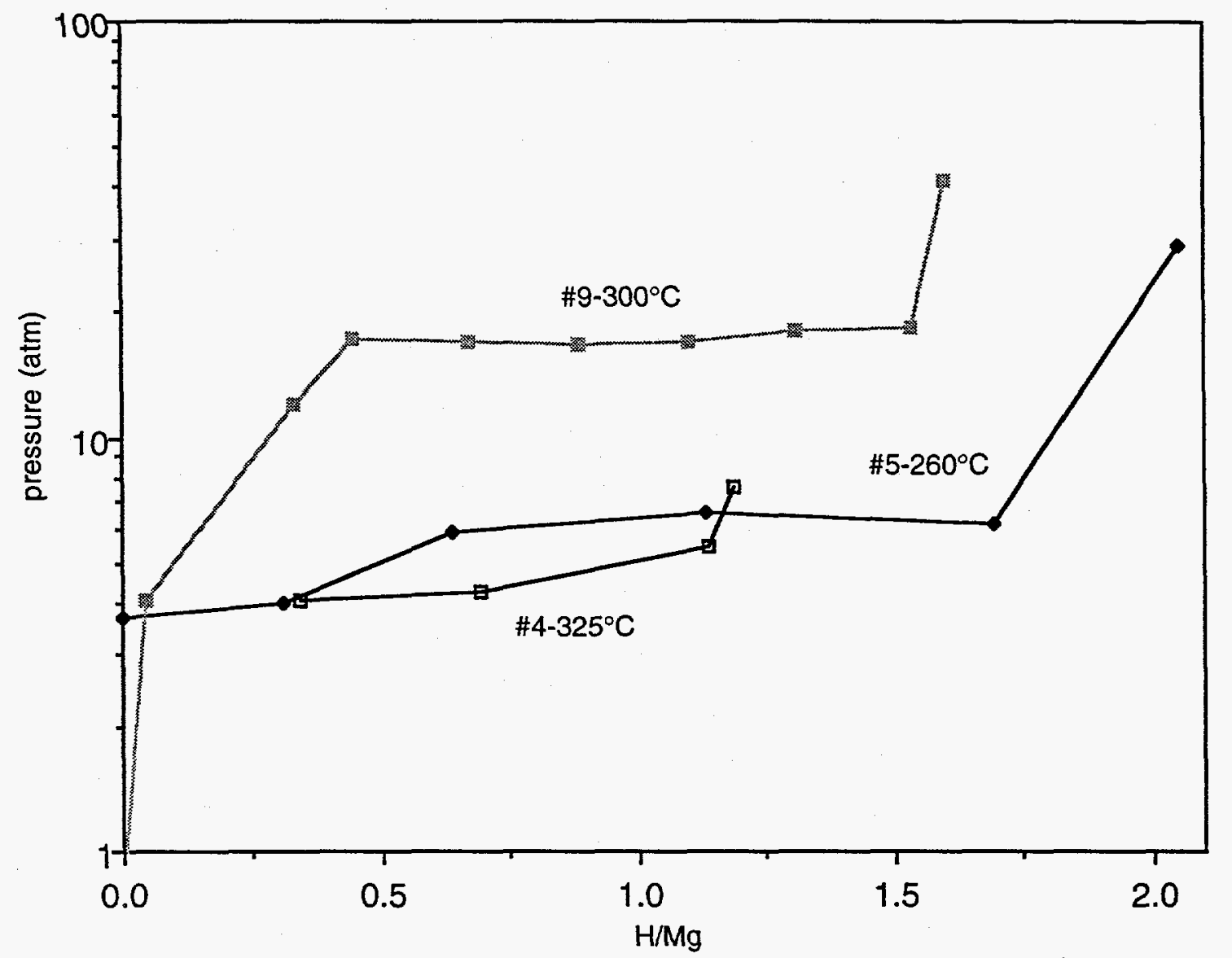

Figure 23. Evolution of plateau pressure in $A Z 61$ alloy. The higher pressure of the \#5 run at $260^{\circ} \mathrm{C}$ indicates that the material has altered its behavior from that of $\# 4$ at $325^{\circ} \mathrm{C}$. Subsequent runs such as $\# 9$ at $300^{\circ} \mathrm{C}$ indicate the irreversibility of the reaction. 


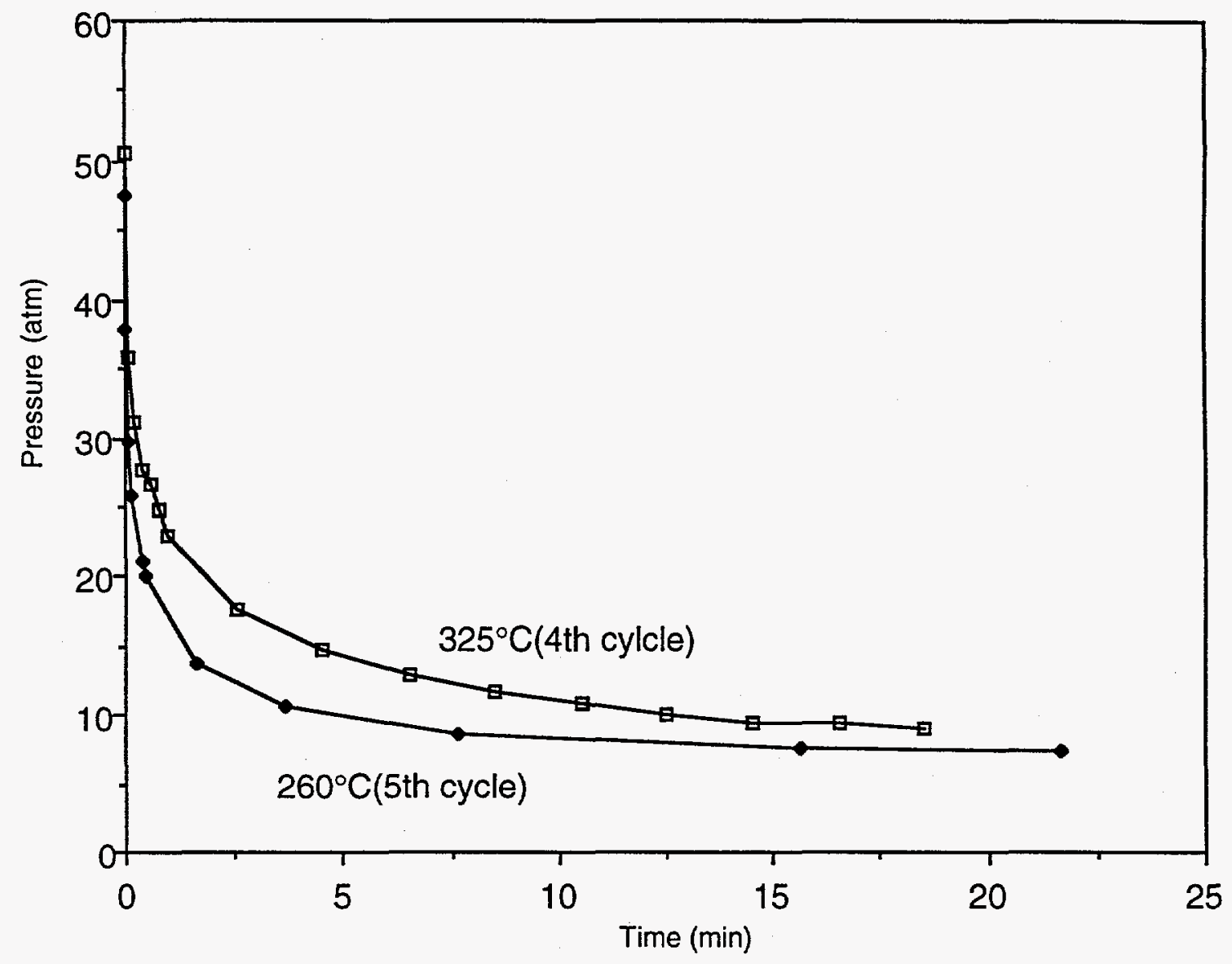

Figure 24. The time to equilibrate on comparable absorption aliquots was also different before and after the transformation of the AZ61 alloy. The new phase was faster than the old phase even though the temperature was lower. 


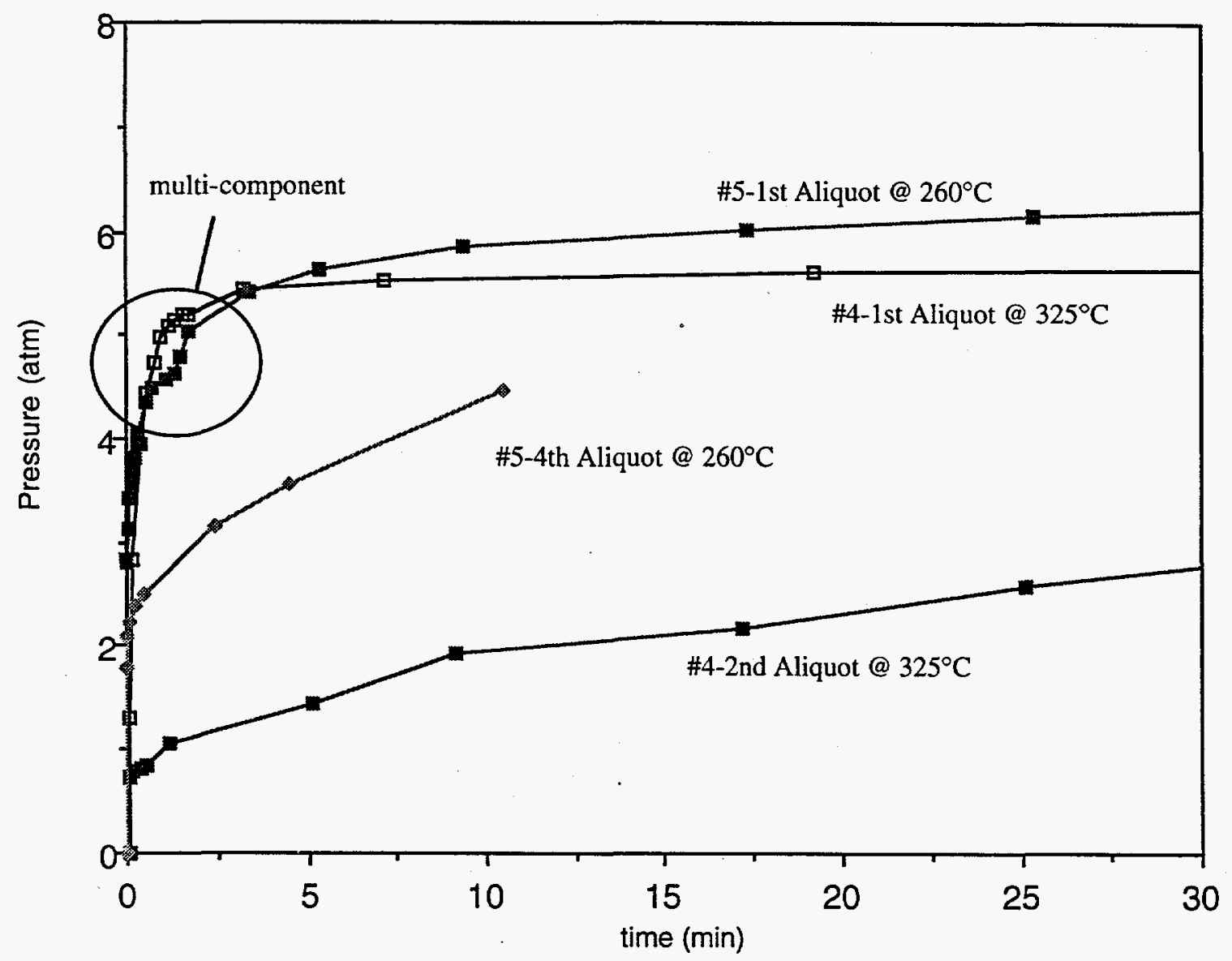

Figure 25. The desorption time differences for different aliquots before and after the transformation in AZ61 alloy also reflected faster kinetics. Later aliquots reflected a greater discrepancy in rate as the 4 th aliquot (\#5) at $260^{\circ} \mathrm{C}$ still exceeded the $2 \mathrm{nd}$ aliquot (\#4) at $325^{\circ} \mathrm{C}$. 


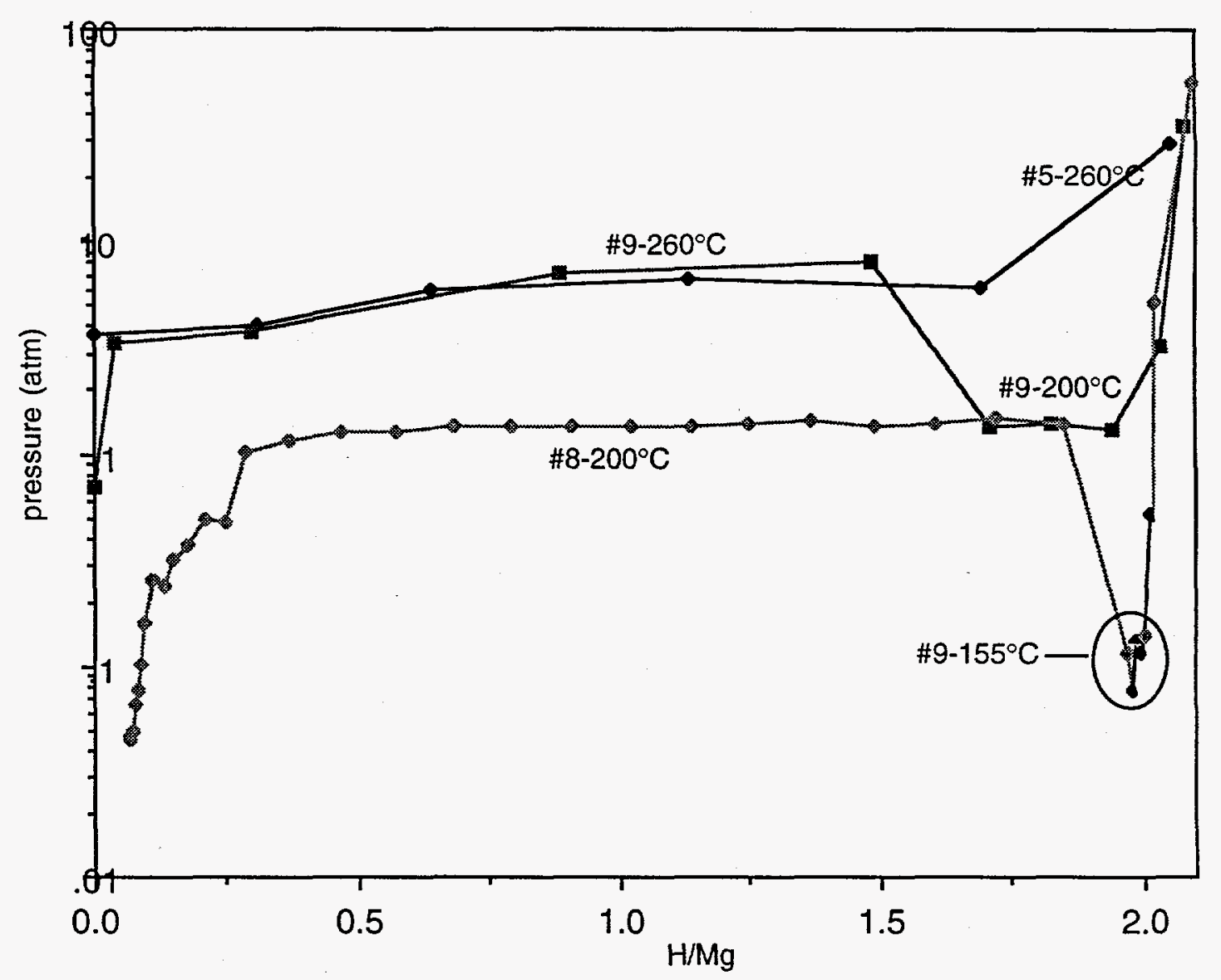

Figure 26. A later run (\#9) was used to test the reproducibility of the plateau values at $200^{\circ} \mathrm{C}$ and $260^{\circ} \mathrm{C}$ after transformation complete in AZ61 alloy. Additional points also indicated kinetic activity at $155^{\circ} \mathrm{C}$. 


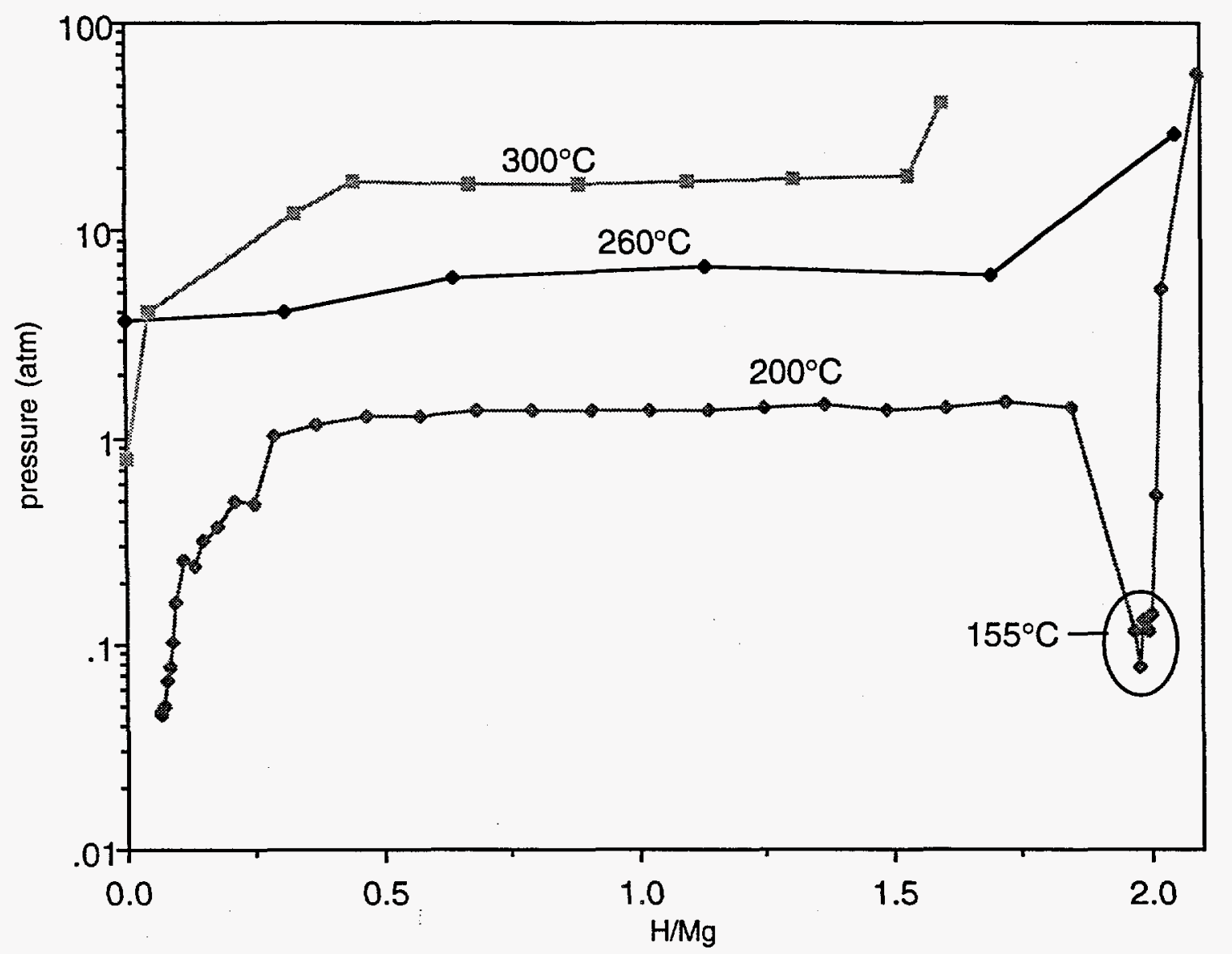

Figure 27 Desorption isotherms are shown for the for AZ61 alloy. Data suggests the possibility of two phases: one at the higher compositions and one at the lower values starting about $0.5 \mathrm{H} / \mathrm{Mg}$. 


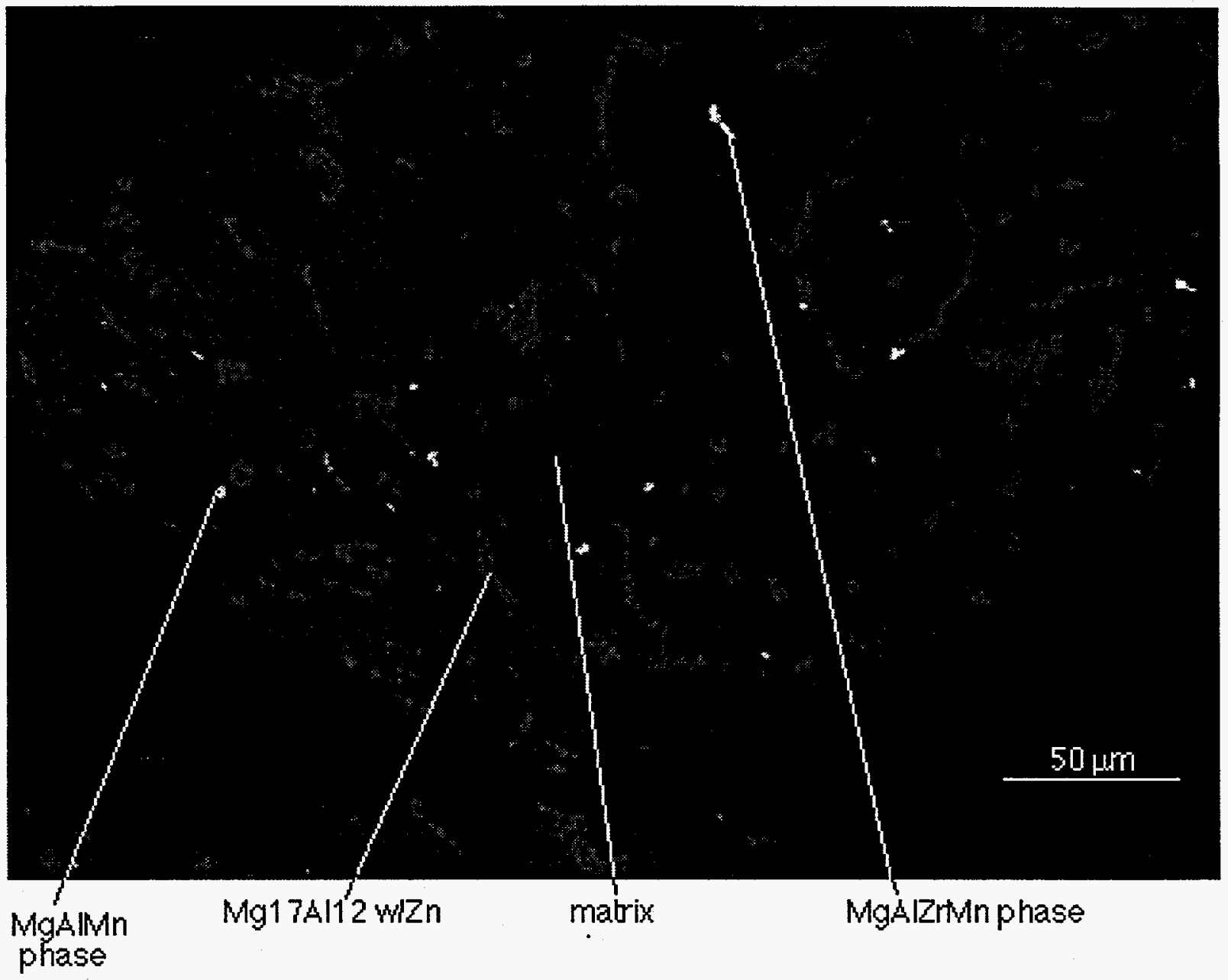

Figure 28. The micrograph of the as-received AZ 91 alloy showed more second phase than the other $\mathrm{AZ}$ alloys. There were additional phases with $\mathrm{Mn}$ and $\mathrm{Zr}$ in large concentrations. 


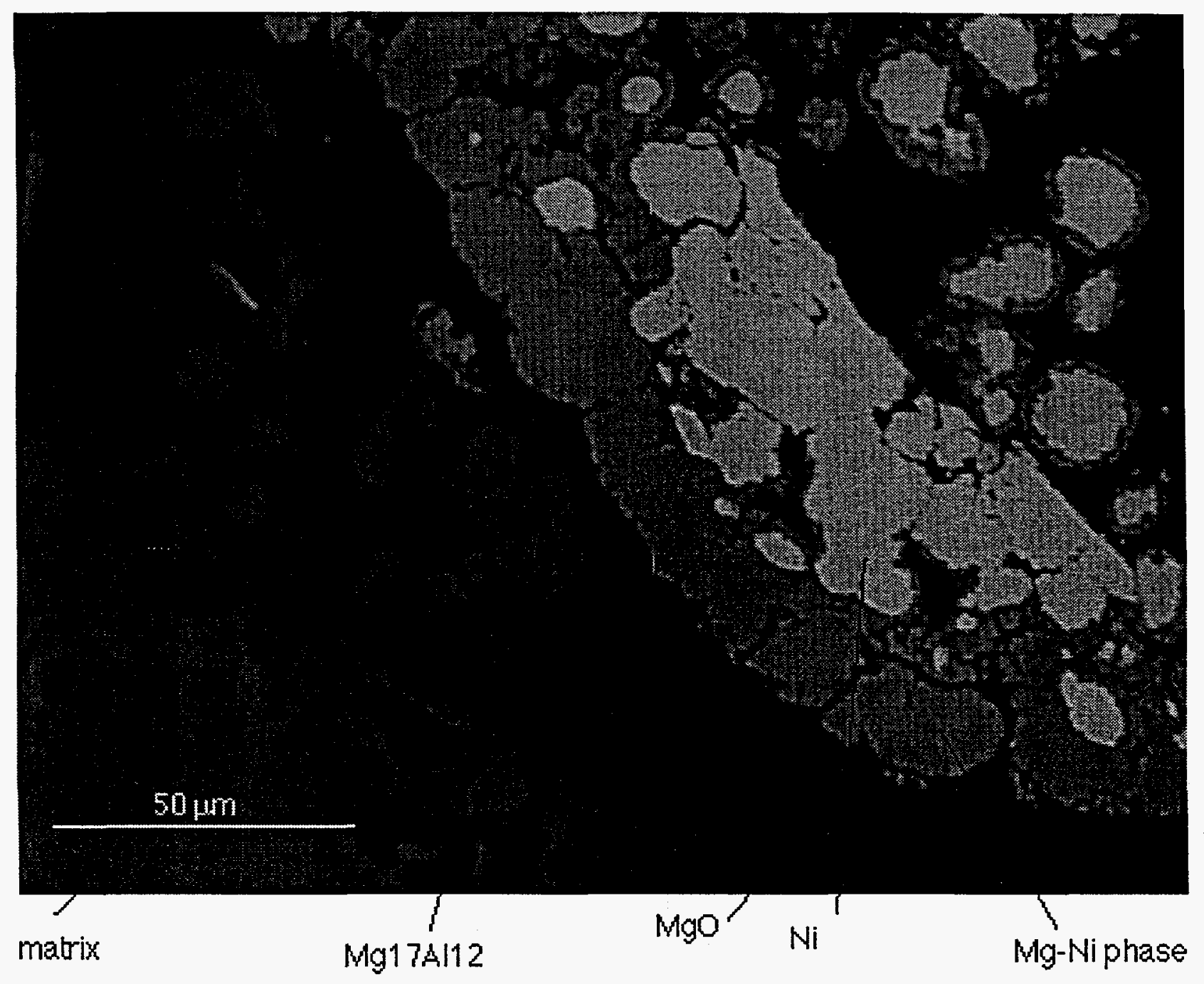

Figure 29. AZ91 alloy after hydriding showed evidence of $\mathrm{Mg} 2 \mathrm{Ni}$ formation in region of near contact between the alloy and the Ni particles. No reaction zone was present. 


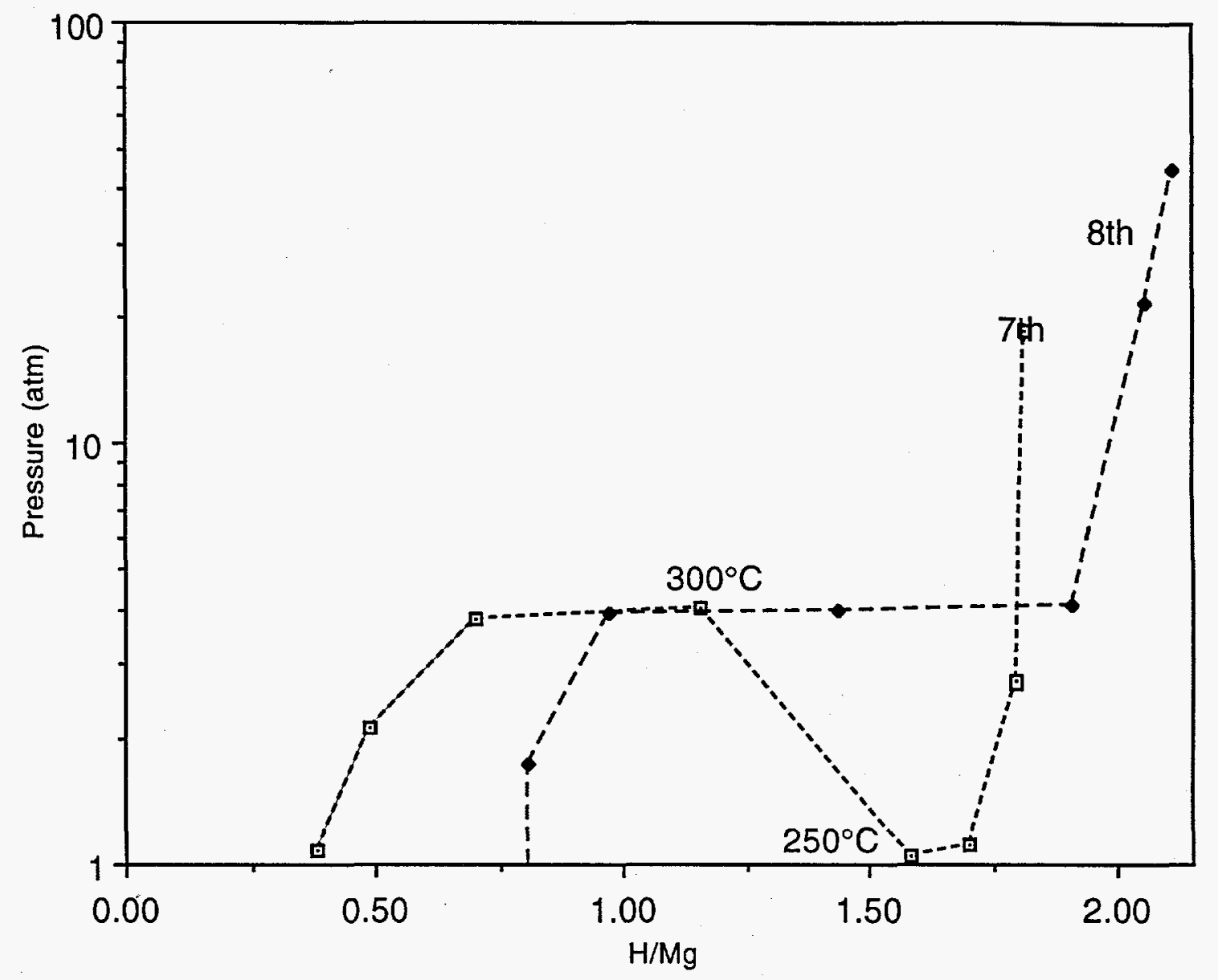

Figure 30. Desorption isotherms for AZ 91 alloy indicated further evolution of a hydride phase that had equilibrium plateau pressures slightly higher than $\mathrm{Mg}_{2} \mathrm{Ni}$. 


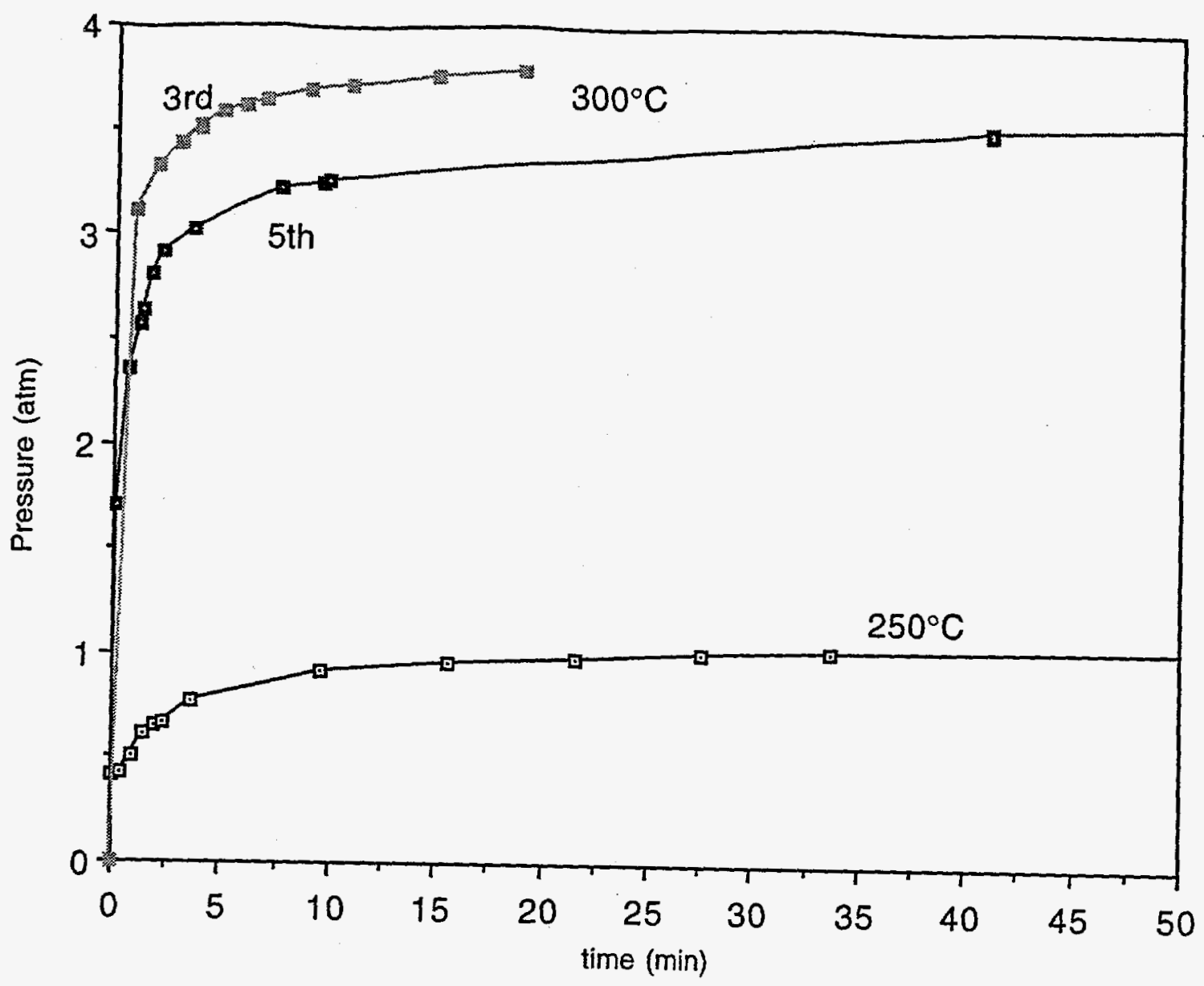

Figure 31. The desorption behavior for AZ 91 alloy exhibited reasonable kinetics at temperatures as low as $250^{\circ} \mathrm{C}$. 


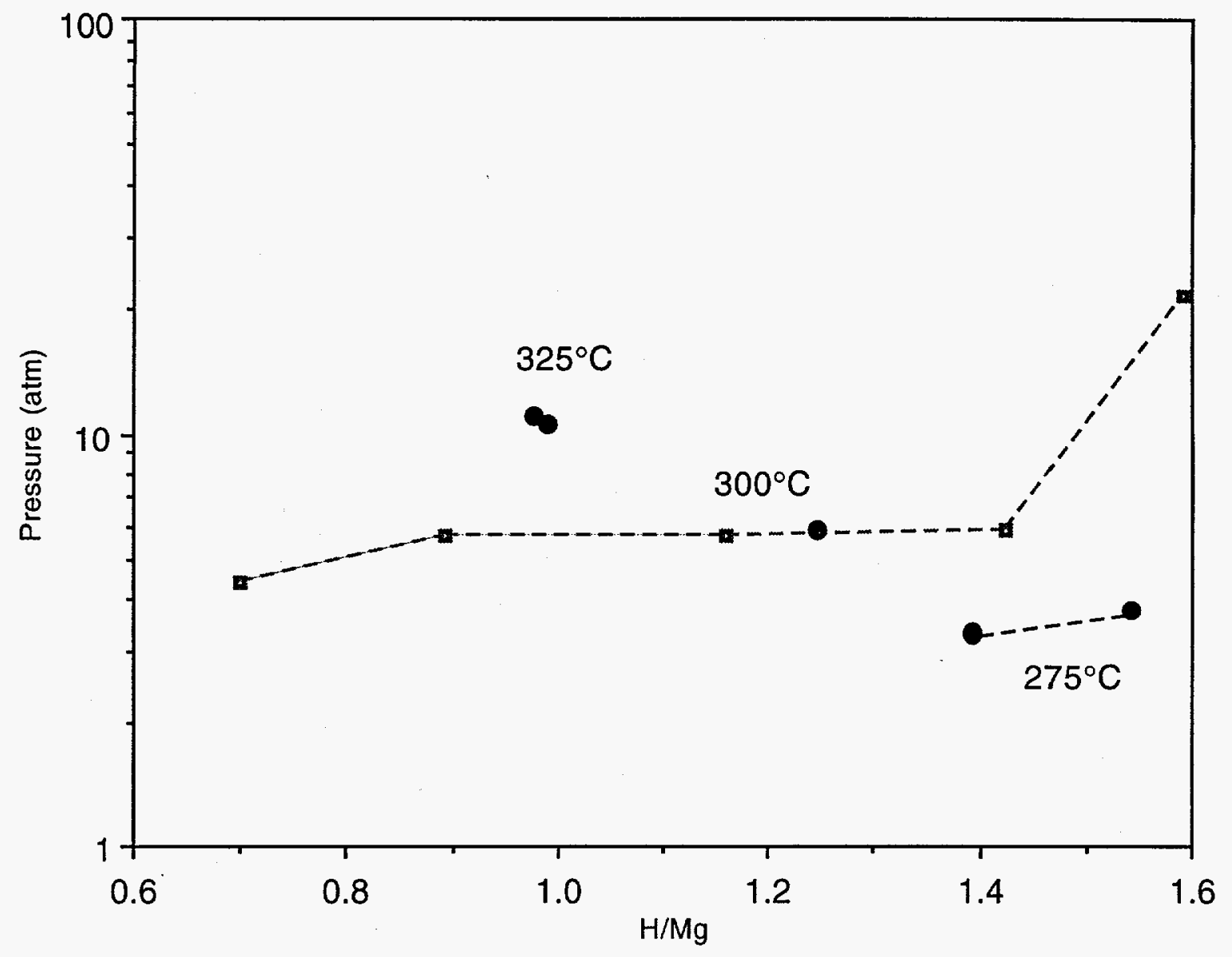

Figure 32. Limited isotherms were measured for the $\mathrm{Mg}_{17} \mathrm{Al}_{12}$ line compound $+\mathrm{Zn}$ due to the slow kinetics of the alloy. 


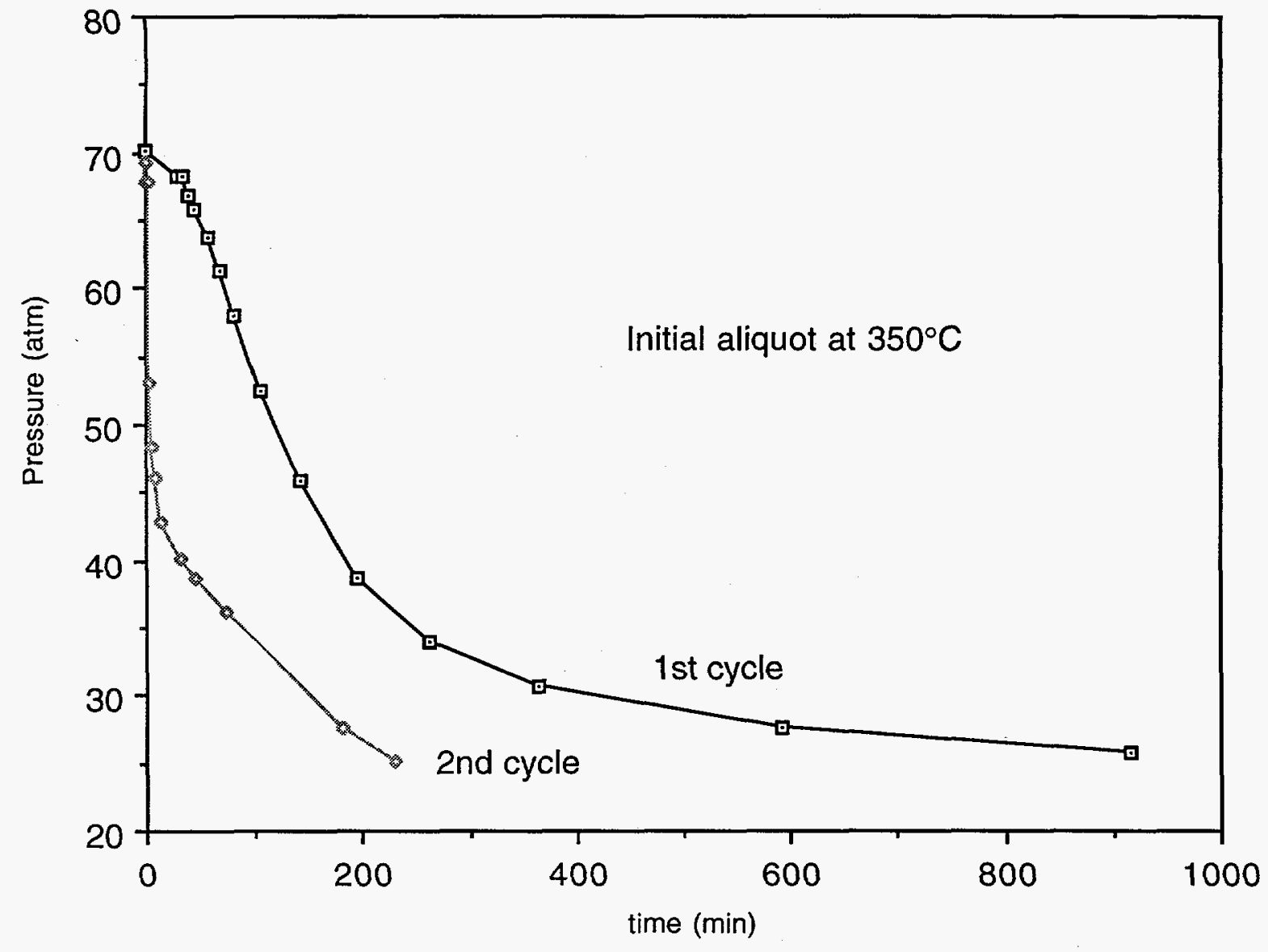

Figure 33. Sigmoidal shape to the initial loading cycle for $\mathrm{Mg}_{17} \mathrm{Al}_{12}$ line compound $+\mathrm{Zn}$ indicates hydrogen assisted transformation may play a role in further activation of this alloy. 


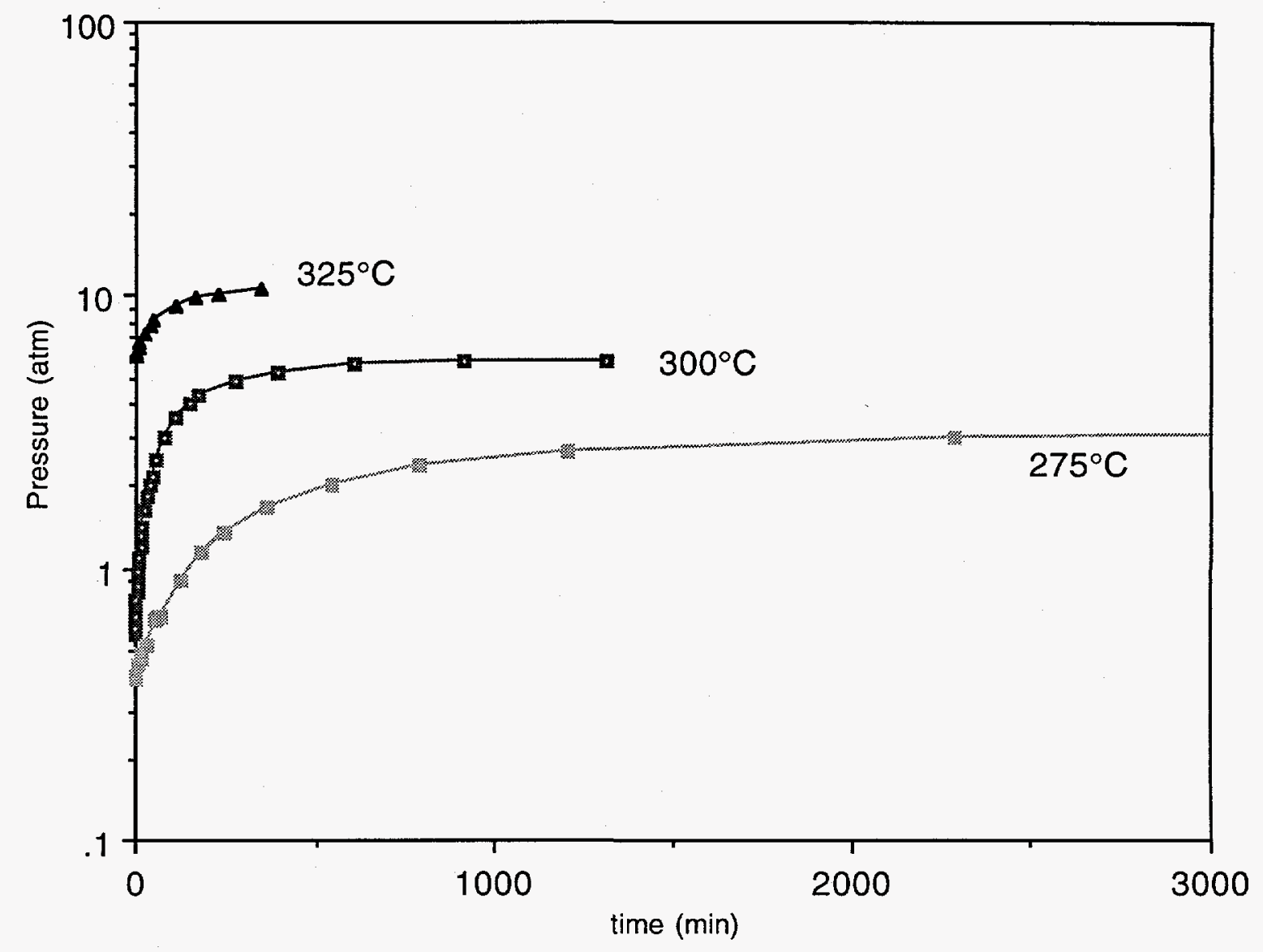

Figure 34. Desorption time per aliquot slowed as the temperature was decreased for the $\mathrm{Mg}_{17} \mathrm{Al}_{12}$ line compound $+\mathrm{Zn}$. 


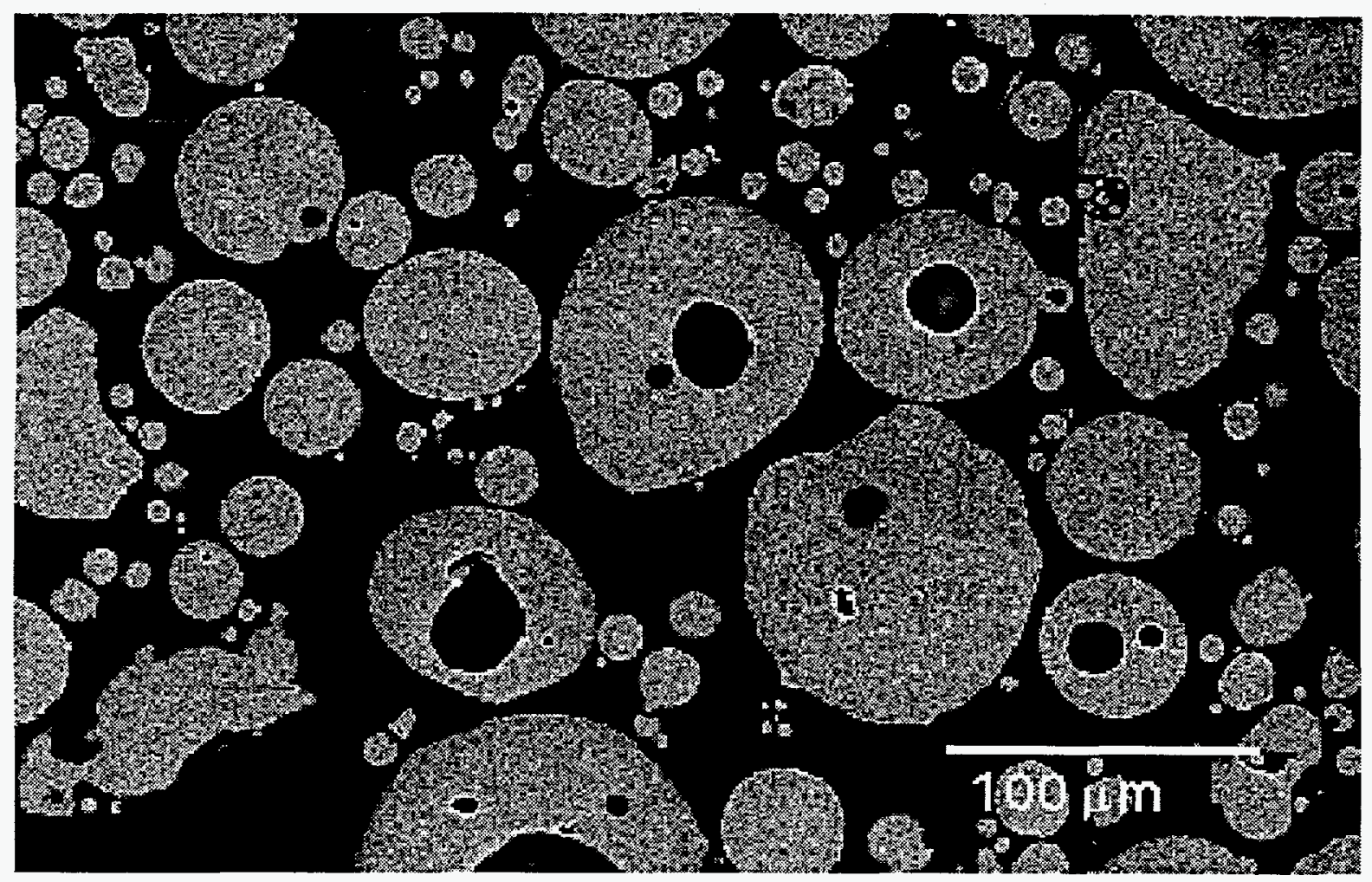

Figure 35. Micrograph reflects the large size distribution and some evidence for voids in gas atomized $\mathrm{Al}_{3} \mathrm{Mg}_{2}$ powders. 


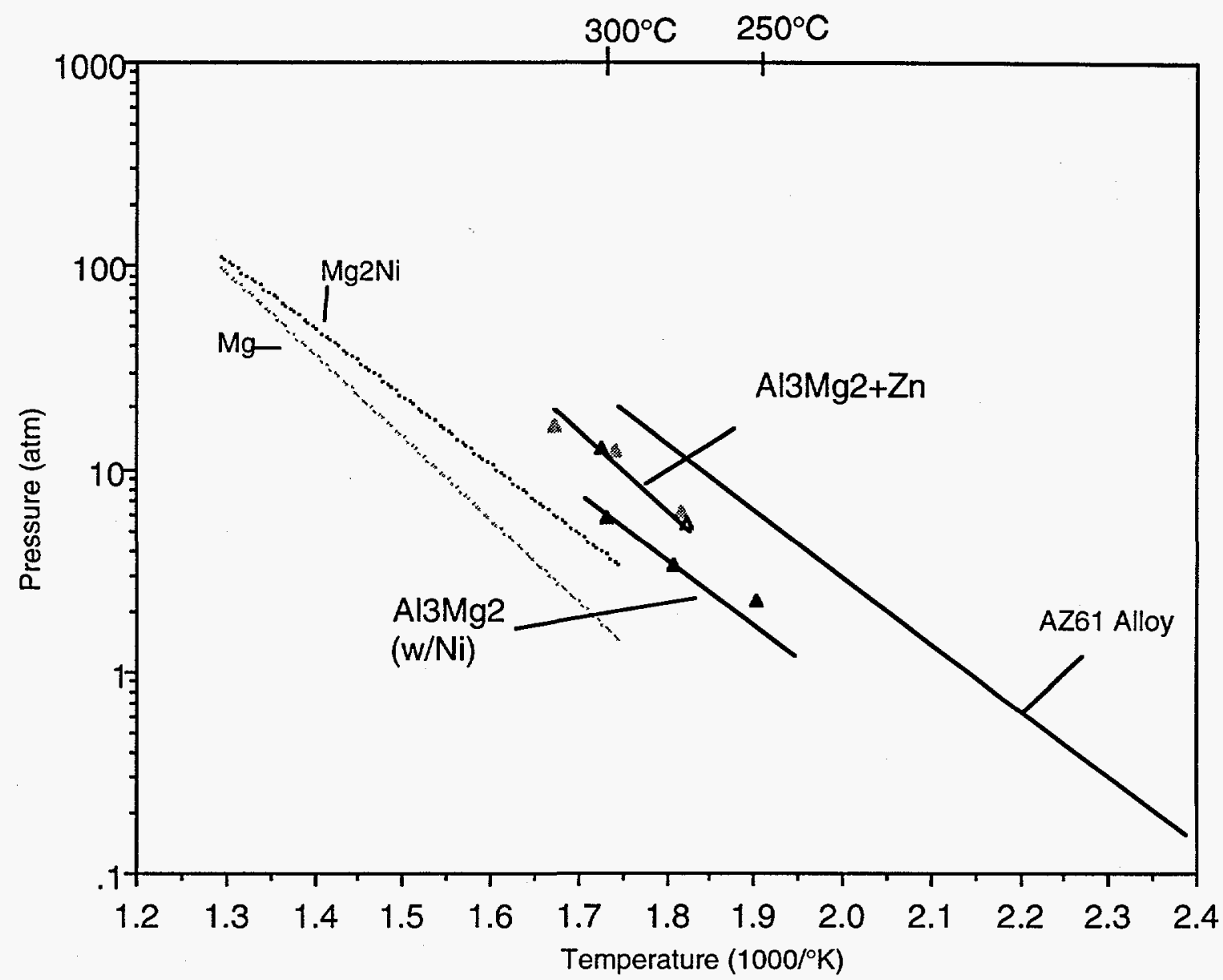

Figure 36. A comparison of the equilibrium van't Hoff plot for $\mathrm{Al}_{3} \mathrm{Mg}_{2}$ line compound with $\mathrm{Ni}$ and $\mathrm{Zn}$ additives to other $\mathrm{Mg}$ and other alloys. 


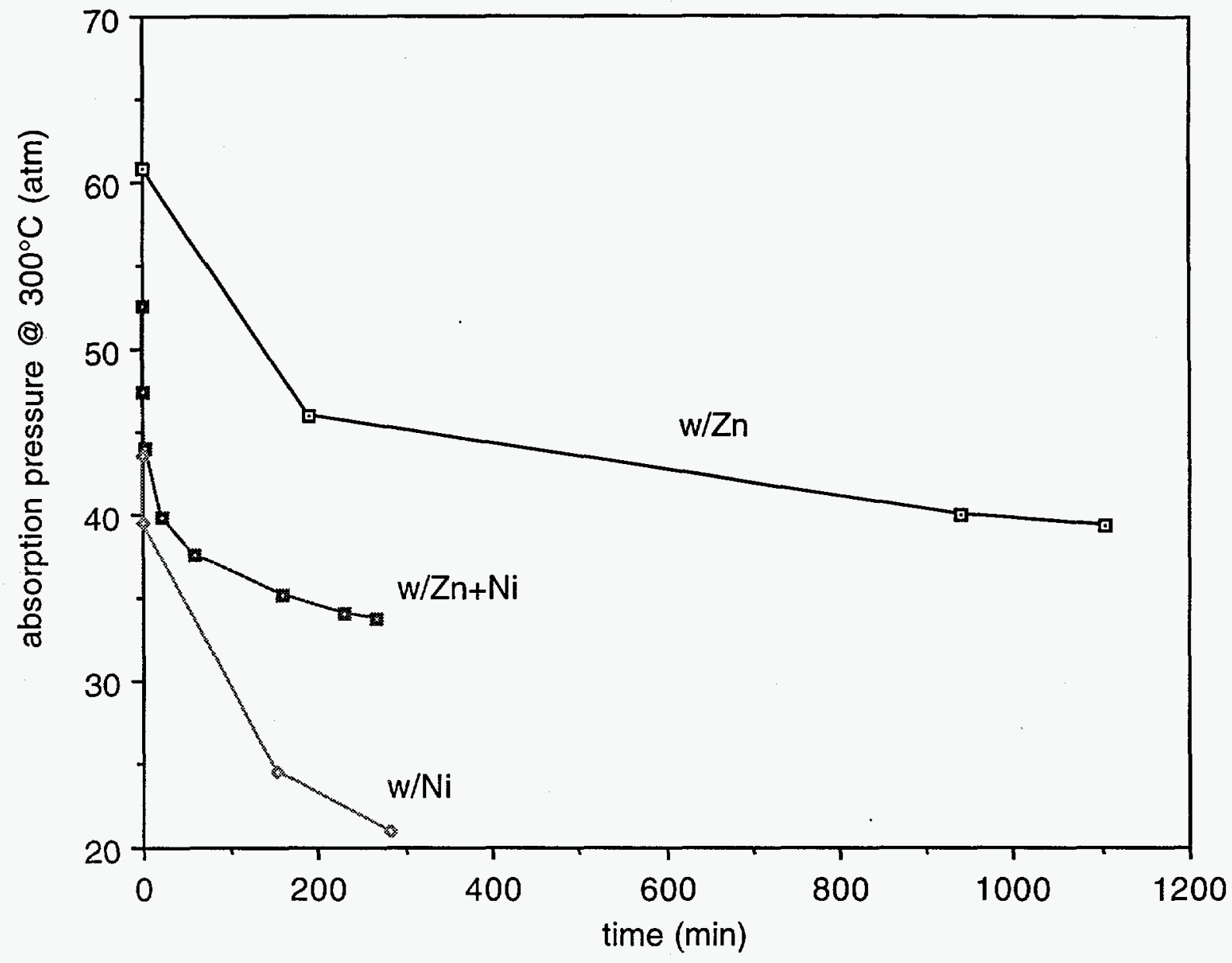

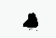

$+$

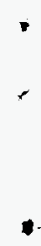

Figure 37. Both $\mathrm{Zn}$ and $\mathrm{Ni}$ additives permitted loading of the $\mathrm{Al}_{3} \mathrm{Mg}_{2}$ line compound. 


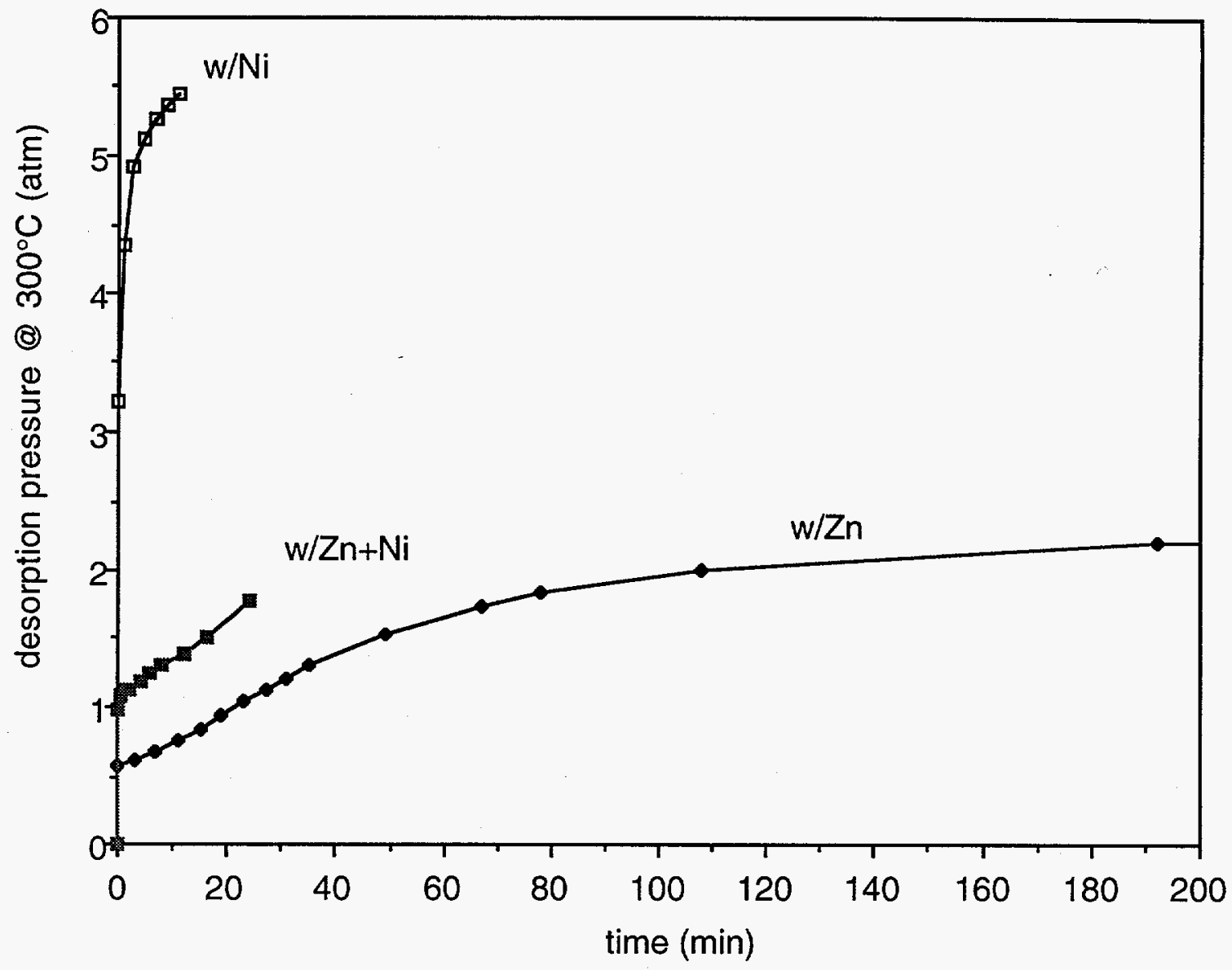

Figure 38. The addition of Ni permitted the fastest desorption equilibria, but higher pressures with $\mathrm{Al}_{3} \mathrm{Mg}_{2}$ were eventually realized with $\mathrm{Zn}$ as an additive. 


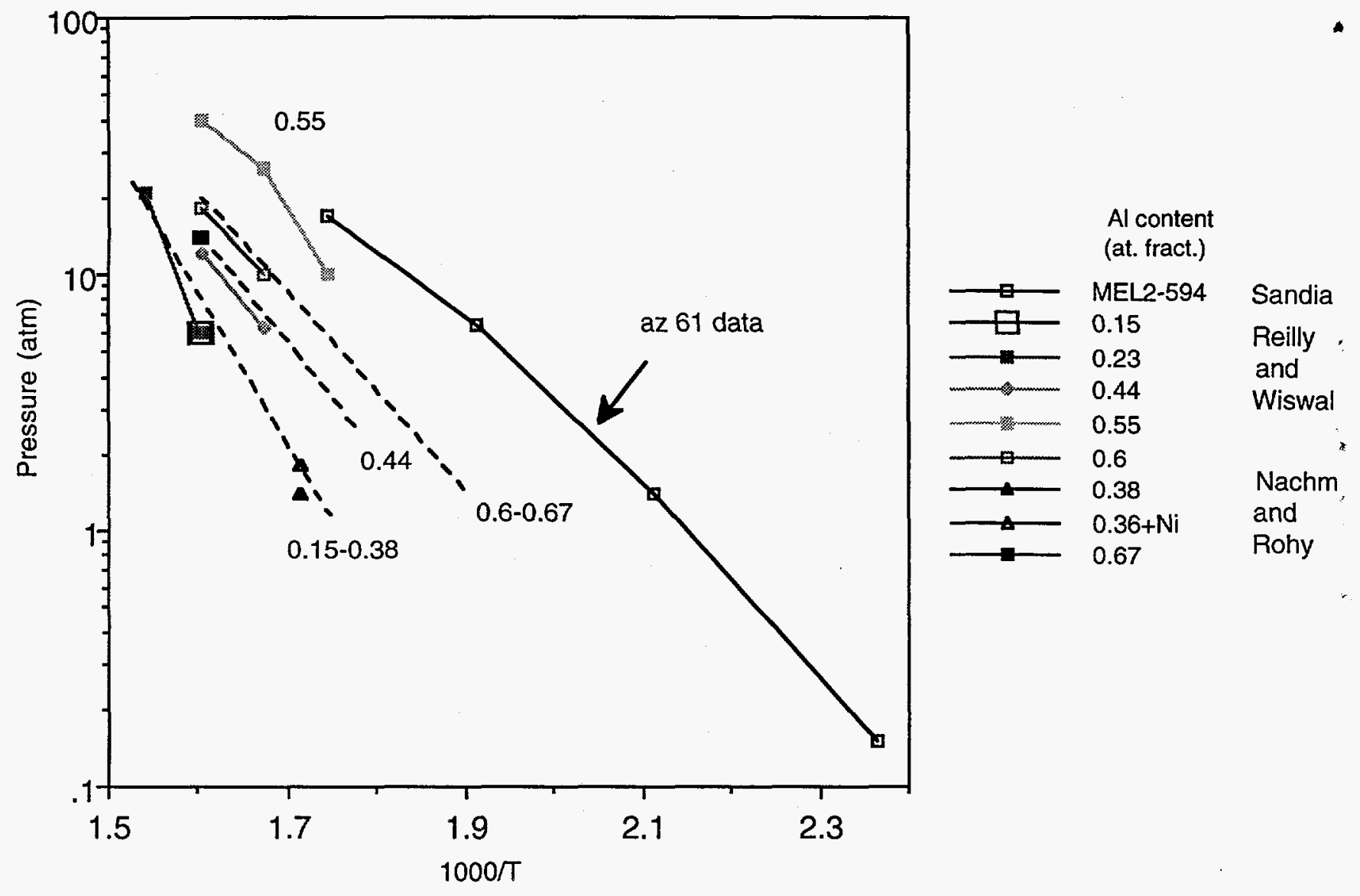

Figure 39. van't Hoff comparison of $A Z 61$ to earlier data suggest that the elemental additions and the formation of multi-phase alloys may be a promising approach to modification of the $\mathrm{Mg}$-Al hydride systems. 


\section{UNLIMITED RELEASE}

\section{INITIAL DISTRIBUTION}

University of California, Irvine

Attn: E. Lavernia

Department of Mechanical

\& Aerospace Engineering

Irvine, CA 92717-3975

MS $0188 \quad$ LDRD

MS 9001 T. O Hunter, 8000

Attn: J. B. Wright, 2200

M. E. John, 8100

L. A. West, 8200

W. J. McLean, 8300

R. C. Wayne, 8400

P. N. Smith, 8500

P. E. Brewer, 8800

D. L. Crawford, 8900

MS $9161 \quad$ D. F. Cowgill, 8716

MS $9161 \quad$ K. L. Wilson, 8716

MS 9163 W. Bauer, 8302 (5)

MS $9161 \quad$ W. G. Wolfer, 8717

MS $9402 \quad$ C. K. Rood, 8715

MS 9402 A. D. Gardea, 8715

MS 9402 M. E. Malinowski, 8715

MS 9402 K. D. Stewart, 8715

MS $9402 \quad$ G. J. Thomas, 8715 (5)

MS 9402 N. Y. C. Yang, 8715 (5)

MS 9403 M. I. Baskes, 8712

MS $9403 \quad$ S. E. Guthrie, 8712 (5)

MS 9403 A. M. Sieber, 8712

MS 9405 M. T. Dyer, 8700

Attn: M. E. Perra, 8711

J. C. F. Wang, 8713

G. A. Benedetti, 8741

M. R. Birnbaum, 8742

P. E. Nielan, 8743

W. A. Kawahara, 8746

MS 9405 J. R. Spingarn, 8230

MS 9021 Technical Communications

Department, 8815, for OSTI (10)
MS 9021 Technical Communications Department, 8815/Technical Library, MS0899, 4414

MS 0899 Technical Library, 4414 (4)

MS 9018 Central Technical Files, 8940-2 (3) 